TRANSACTIONS OF THE

AMERICAN MATHEMATICAL SOCIETY

Volume 354, Number 9 , Pages 3475-3534

S 0002-9947(02)03025-8

Article electronically published on April 30, 2002

\title{
WEYL-TITCHMARSH $M$-FUNCTION ASYMPTOTICS, LOCAL UNIQUENESS RESULTS, TRACE FORMULAS, AND BORG-TYPE THEOREMS FOR DIRAC OPERATORS
}

\author{
STEVE CLARK AND FRITZ GESZTESY
}

Dedicated to F. V. Atkinson, one of the pioneers of this subject

\begin{abstract}
We explicitly determine the high-energy asymptotics for WeylTitchmarsh matrices associated with general Dirac-type operators on half-lines and on $\mathbb{R}$. We also prove new local uniqueness results for Dirac-type operators in terms of exponentially small differences of Weyl-Titchmarsh matrices. As concrete applications of the asymptotic high-energy expansion we derive a trace formula for Dirac operators and use it to prove a Borg-type theorem.
\end{abstract}

\section{INTRODUCTION}

The high-energy asymptotics, $|z| \rightarrow \infty$, of scalar-valued Weyl-Titchmarsh functions, $m_{+}\left(z, x_{0}\right)$, associated with general half-line Dirac-type differential expressions of the form

$$
J \frac{d}{d x}-B(x), \quad J=\left(\begin{array}{cc}
0 & -1 \\
1 & 0
\end{array}\right)
$$

and $B$ a self-adjoint $2 \times 2$ matrix with real-valued coefficients, $B^{(n)} \in L^{1}\left(\left[x_{0}, c\right]\right)^{2 \times 2}$ for some $n \in \mathbb{N}_{0}(=\mathbb{N} \cup\{0\})$ and all $c>x_{0}$, received quite a bit of attention over the past two decades, as can be inferred, for instance, from [33], [57, 61, 62], [98. (and the literature therein). Hence, it may perhaps come as a surprise that the corresponding matrix extension of this problem, considering general matrix-valued differential expressions of the type

$$
J \frac{d}{d x}-B(x), \quad J=\left(\begin{array}{cc}
0 & -I_{m} \\
I_{m} & 0
\end{array}\right)
$$

with $I_{m}$ the identity matrix in $\mathbb{C}^{m}, m \in \mathbb{N}$, and $B$ a self-adjoint $2 m \times 2 m$ matrix satisfying $B^{(n)} \in L^{1}\left(\left[x_{0}, c\right]\right)^{2 m \times 2 m}$ for some $n \in \mathbb{N}_{0}$ and all $c>x_{0}$, apparently, received no attention at all. (It should be noted that this observation discounts papers in the special scattering-theoretic case concerned with short-range coefficients $B^{(n)} \in L^{1}\left(\left[x_{0}, \infty\right) ;(1+|x|) d x\right)^{2 m \times 2 m}$, where iterations of Volterra-type integral equations yield the asymptotic high-energy expansion of $M_{+}\left(z, x_{0}\right)$ as $|z| \rightarrow \infty$ to any order, cf. Lemma 4.1.) This is not because of a lack of interest in this type

Received by the editors February 15, 2002.

2000 Mathematics Subject Classification. Primary 34B20, 34E05, 34L40; Secondary 34A55.

Key words and phrases. Weyl-Titchmarsh matrices, high-energy expansions, uniqueness results, trace formulas, Borg theorems, Dirac operators.

Supported in part by NSF grant INT-9810322. 
of problem (we will discuss its relevance below), but simply since it is a nontrivial one, which, in many of its aspects, must be regarded as more difficult than the corresponding matrix-valued Schrödinger operator case, which, in turn, was only very recently settled in [20]. The results proven in this paper show that in leading order (and independently of the self-adjoint boundary condition chosen at $x_{0}$ ),

$$
M_{+}\left(z, x_{0}\right) \underset{\substack{|z| \rightarrow \infty \\ z \in C_{\varepsilon}}}{=} i I_{m}+o(1),
$$

where $C_{\varepsilon}$ denotes the open sector in the open upper complex half-plane $\mathbb{C}_{+}$with vertex at zero, symmetry axis along the positive imaginary axis, and opening angle $\varepsilon$, with $0<\varepsilon<\pi / 2$. We are interested in proving the asymptotic expansion (1.3), and especially in its higher-order analogs in powers of $1 / z$, under optimal smoothness hypotheses on $B$. Such results are then also derived for the $2 m \times 2 m$ analog $M(z, x)$ of $M_{+}(z, x)$ associated with Dirac-type operators on $\mathbb{R}$.

Our principal motivation in studying this problem stems from our general interest in operator-valued Herglotz functions (cf. [17], 41], 42], 44], 45], 46], [47, [52], [114]) and their possible applications in the areas of inverse spectral theory and completely integrable systems. More precisely, using higher-order asymptotic expansions of $M_{+}(z, x)$, one can prove trace formulas for $B(x)$ and certain higherorder differential polynomials in $B(x)$ (similar in spirit to an approach pioneered in 49] (see also [38, 40]) in connection with Schrödinger operators). These trace formulas, in turn, then can be used to prove various results in inverse spectral theory for matrix-valued Dirac-type operators $D=J \frac{d}{d x}-B$ in $L^{2}(\mathbb{R})^{2 m}$. For instance, using one of the principal results of this paper, Theorem 4.7, and its straightforward application to the asymptotic high-energy expansion of the diagonal Green's matrix $G(z, x, x)=(D-z)^{-1}(x, x)$ of $D$, the following matrix-valued analog of a classical uniqueness result of Borg [15] for one-dimensional Schrödinger operators will be proven in the context of Dirac-type operators in Section 6 .

Theorem 1.1. Suppose that $B$ is of the normal form

$$
B(x)=\left(\begin{array}{cc}
B_{1,1}(x) & B_{1,2}(x) \\
B_{1,2}(x) & -B_{1,1}(x)
\end{array}\right),
$$

with $B_{1,1}(x)$ and $B_{1,2}(x)$ self-adjoint for a.e. $x \in \mathbb{R}$, and assume that $D$ is reflectionless (e.g., $B$ is periodic and $D$ has uniform spectral multiplicity $2 m$ ). In addition, suppose that $D$ has spectrum equal to $\mathbb{R}$. Then,

$$
B(x)=0 \quad \text { for a.e. } x \in \mathbb{R} .
$$

For related results see, for instance, 1], 2], 23, 37, 48, [54, 56]. Incidentally, the higher-order differential polynomials in $B(x)$ just alluded to represent the Ablowitz-Kaup-Newell-Segur (AKNS) or Zakharov-Shabat (ZS) invariants (i.e., densities associated with the AKNS-ZS conservation laws) and hence provide a link to infinite-dimensional completely integrable systems (cf., e.g., [7], [18], [28], [29], 30], [91, 95], [109], 112], [111], [113], and the references therein), especially, hierarchies of matrix-valued (i.e., nonabelian) nonlinear Schrödinger equations.

Although various aspects of inverse spectral theory for scalar Schrödinger, Jacobi, and Dirac-type operators, and more generally, for $2 \times 2$ Hamiltonian systems, are well-understood by now (cf. the extensive list of references provided in [42]), the corresponding theory for such operators and Hamiltonian systems with $m \times m$ matrix-valued coefficients, $m \in \mathbb{N}$, is still in its infancy. A particular inverse spectral 
theory aspect we have in mind is that of determining isospectral sets (manifolds) of such systems. It may, perhaps, come as a surprise that determining the isospectral set of Hamiltonian systems with matrix-valued periodic coefficients is a completely open problem. It appears to be no exaggeration to claim that absolutely nothing seems to be known about the corresponding isospectral sets of periodic Dirac-type operators in the case $m \geq 2$. (More or less the same ignorance applies to Schrödinger, Jacobi, and more generally, to periodic $2 m \times 2 m$ Hamiltonian systems with $m \geq 2$.) Theorem 1.1 can be viewed as a first (and very modest) step toward the construction of isospectral manifolds of certain classes of matrix-valued potential coefficients $B$ for Dirac-type operators.

However, asymptotic high-energy expansions for Weyl-Titchmarsh matrices on half-lines and on $\mathbb{R}$, their applications to trace formulas for $B(x)$, and the derivation of Borg-type theorems for Dirac operators are not the only topics under consideration in this paper. We also provide a comprehensive and new treatment of local uniqueness theorems for $B$ in terms of exponentially close Weyl-Titchmarsh matrices. More precisely, in Section [5] we will prove the following result $\left(\|\cdot\|_{\mathbb{C}^{m \times m}}\right.$ denotes a matrix norm on $\left.\mathbb{C}^{m \times m}\right)$.

Theorem 1.2. Fix $x_{0} \in \mathbb{R}$ and suppose that $B_{j} \in L^{1}\left(\left[x_{0}, x_{0}+R\right]\right)^{2 m \times 2 m}$ for all $R>0$, posseses the normal form given in Theorem 1.1 a.e. on $\left(x_{0}, \infty\right), j=1,2$. Denote by $M_{j,+}(z, x), x \geq x_{0}$, the unique Weyl-Titchmarsh matrices corresponding to the half-line Dirac-type operators in $L^{2}\left(\left[x_{0}, \infty\right)\right)^{2 m}$ associated with $B_{j}, j=1,2$ (fixing some self-adjoint boundary condition at $x_{0}$ ). Then,

$$
\text { if for some } a>0, B_{1}(x)=B_{2}(x) \text { for a.e. } x \in\left(x_{0}, x_{0}+a\right) \text {, }
$$

one obtains

$$
\left\|M_{1,+}\left(z, x_{0}\right)-M_{2,+}\left(z, x_{0}\right)\right\|_{\mathbb{C}^{m \times m}} \underset{\substack{|z| \rightarrow \infty \\ z \in \rho_{+}}}{=} O\left(e^{-2 \operatorname{Im}(z) a}\right)
$$

along any ray $\rho_{+} \subset \mathbb{C}_{+}$with $0<\arg (z)<\pi$ (and for all self-adjoint boundary condition at $\left.x_{0}\right)$. Conversely, if $m>1$, assume in addition that

$$
B_{j} \in L^{\infty}\left(\left[x_{0}, x_{0}+a\right]\right)^{2 m \times 2 m}, \quad j=1,2 .
$$

Moreover, suppose that for all $\varepsilon>0$,

$$
\left\|M_{1,+}\left(z, x_{0}\right)-M_{2,+}\left(z, x_{0}\right)\right\|_{\mathbb{C}^{m \times m}} \underset{\substack{|z| \rightarrow \infty \\ z \in \rho_{+, \ell}}}{=} O\left(e^{-2 \operatorname{Im}(z)(a-\varepsilon)}\right), \quad \ell=1,2,
$$

along a ray $\rho_{+, 1} \subset \mathbb{C}_{+}$with $0<\arg (z)<\pi / 2$ and along a ray $\rho_{+, 2} \subset \mathbb{C}_{+}$with $\pi / 2<\arg (z)<\pi$. Then

$$
B_{1}(x)=B_{2}(x) \quad \text { for a.e. } x \in\left[x_{0}, x_{0}+a\right] .
$$

We also prove the analog of Theorem 1.2 for the $2 m \times 2 m$ Weyl-Titchmarsh matrices $M_{j}(z, x)$ associated with Dirac-type operators on $\mathbb{R}$ corresponding to $B_{j}$, $j=1,2$.

In the context of scalar Schrödinger operators, the analog of Theorem 1.2 was first proved by Simon [115]. An alternative proof, applicable to matrix-valued Schrödinger operators, was presented in [51] (cf. also [42]). More recently, yet another proof was found by Bennewitz [13] (following some ideas in [16]). In fact, our proof of Theorem 1.2 is based on that of Bennewitz [13] with additional modifications necessary to accomodate Dirac-type operators. These results extend the 
classical (global) uniqueness results due to Borg [16] and Marchenko [92, 93] which state that half-line $m$-functions uniquely determine the corresponding potential coefficient. The Dirac-type results such as Theorem 1.2 appear to be new, even in the special case $m=1$. Previous results in the Dirac case focused on global uniqueness questions only. We refer to Gasymov and Levitan [35] in the case $m=1$ and to Lesch and Malamud 82 in the matrix case $m \in \mathbb{N}$.

Next, we briefly sketch the content of each section. Section 2 provides the necessary background results on Dirac-type operators and recalls the basic notions of Weyl-Titchmarsh theory for Hamiltonian systems on a half-line as well as on $\mathbb{R}$, as developed in detail by Hinton and Shaw in a series of papers [63 - 67] (see also [8], 72], 68], 69], 74, 75], 79], 80, 81, 83], 100, 111]). In fact, most of these references deal with more general singular Hamiltonian systems, and hence we specialize some of this material to the Dirac-type operator case at hand. While our treatment of Weyl-Titchmarsh theory in Section 2 is somewhat detailed, the results presented appear to be of vital importance for our asymptotic expansions in Sections 3 and 4 . At any rate, we intended to present this material as concisely as possible.

Section 3 is devoted to a proof of the leading-order for the asymptotic high-energy expansion (1.3) of $M_{+}(z, x)$ for the Dirac case. We follow the strategy developed in the context of matrix-valued Schrödinger operators in our joint paper [20] by appealing to the theory of Riccati equations. By doing so, we follow the lead of Atkinson, who highlighted the importance of Riccati equations, in this regard, first in [9, subsequently in [10, 11] and ultimately in the unpublished manuscript [12] in which he obtains the leading order for the asymptotic high-energy expansion of $M_{+}(z, x)$ for the matrix-valued Schrödinger case.

Theorems 3.4 and 3.6 contain two characterizations of the Weyl disk (cf. Definition [2.7). These characterizations provide an answer in Remark [3.7] to a point raised in [20] concerning the nature of the Weyl disk. From these characterizations of the Weyl disk, we obtain a realization of $M_{+}(z, x)$ as a differentiable function of $x$ which satisfies a certain Riccati equation globally and whose imaginary part is strictly positive. We observe, in Remark 3.5. that the totality of Weyl disks, $D_{+}(z, x)$ (cf. Defintion 2.12), represents the phase space for these solutions. Thus, the asymptotic expansion we seek, represents the asymptotic high-energy behavior for certain solutions of a given Riccati equation.

Section 4 develops a systematic higher-order high-energy asymptotic expansion of $M_{+}(z, x)$ as $|z| \rightarrow \infty$, combining the leading-order asymptotic result in Section 3 with matrix-valued extensions of some methods based again on an associated Riccati equation. More precisely, following a technique in [50] in the scalar Schrödinger operator context, we show how to derive the general high-energy asymptotic expansion of $M_{+}(z, x)$ as $|z| \rightarrow \infty$ by combining Atkinson's leading-order term in (1.3) and the corresponding asymptotic expansion of $M_{+}(z, x)$ in the special case where $B$ has compact support. Section $[5$ then contains our new local uniqueness results for $B(x)$ in terms of exponentially small differences of Weyl-Titchmarsh matrices as indicated in Theorem [1.2. Finally, in Section [6] we derive a new trace formula for Dirac-type operators $D$ in $L^{2}(\mathbb{R})^{2 m}$, using appropriate Herglotz representation results for the diagonal Green's matrix $G(z, x, x)$ discussed in Section 2 . Moreover, we derive the Borg-type Theorem 1.1 for Dirac operators and close with an application to the case of periodic potential coefficients $B$. 


\section{Weyl-Titchmarsh Matrices for Hamiltonian Systems}

We now turn to the Weyl-Titchmarsh theory for Hamiltonian systems as developed by Hinton and Shaw in a series of papers devoted to the spectral theory of (singular) Hamiltonian systems 63. 67] (see also [68, 69, 80, 81, [106, 111, [112], [113]). Throughout this paper all matrices will be considered over the field of complex numbers $\mathbb{C}$. The basic assumptions throughout are described in the following three hypotheses.

Hypothesis 2.1. Fix $m \in \mathbb{N}$ and define the $2 m \times 2 m$ matrix

$$
J=\left(\begin{array}{cc}
0 & -I_{m} \\
I_{m} & 0
\end{array}\right)
$$

where $I_{m}$ denotes the identity matrix in $\mathbb{C}^{m \times m}$. Suppose

$$
A_{j, k}, B_{j, k} \in L_{\mathrm{loc}}^{1}(\mathbb{R})^{m \times m}, \quad j, k=1,2,
$$

and assume

$$
\begin{aligned}
& A(x)=\left(\begin{array}{ll}
A_{1,1}(x) & A_{1,2}(x) \\
A_{2,1}(x) & A_{2,2}(x)
\end{array}\right) \geq 0, \\
& B(x)=\left(\begin{array}{ll}
B_{1,1}(x) & B_{1,2}(x) \\
B_{2,1}(x) & B_{2,2}(x)
\end{array}\right)=B(x)^{*}
\end{aligned}
$$

for a.e. $x \in \mathbb{R}$.

$L_{\text {loc }}^{1}(\mathbb{R})$ denotes the set of locally integrable functions on $\mathbb{R}$. With $M \in \mathbb{C}^{m \times m}$, let $M^{t}$ denote the transpose, let $M^{*}$ denote the adjoint or conjugate transpose of the matrix $M$ and let $M \geq 0$ and $M \leq 0$ denote nonnegative and nonpositive matrices $M$ (i.e., positive and negative semidefinite matrices). Moreover, $\operatorname{let} \operatorname{Im}(M)=$ $\left(M-M^{*}\right) /(2 i)$ and $\operatorname{Re}(M)=\left(M+M^{*}\right) / 2$ denote, respectively, the imaginary and real parts of the matrix $M$.

Under Hypothesis 2.1 our Hamiltonian system is given by

$$
J \Psi^{\prime}(z, x)=(z A(x)+B(x)) \Psi(z, x), \quad z \in \mathbb{C},
$$

for a.e. $x \in \mathbb{R}$, where $z$ plays the role of the spectral parameter, and where

$$
\Psi(z, x)=\left(\begin{array}{l}
\psi_{1}(z, x) \\
\psi_{2}(z, x)
\end{array}\right), \quad \psi_{j}(z, \cdot) \in A C_{\mathrm{loc}}(\mathbb{R})^{m \times r}, j=1,2 .
$$

$A C_{\text {loc }}(\mathbb{R})$ denotes the set of locally absolutely continuous functions on $\mathbb{R}$. The parameter $r$ in (2.2b) will be context dependent, with $1 \leq r \leq m$.

For our discussions of the Weyl-Titchmarsh theory for the Hamiltonian system (2.2), we introduce the definiteness assumption found in Atkinson [8].

Hypothesis 2.2. For all nontrivial solutions $\Psi$ of (2.2a ) with $r=1$ in (2.2b), we assume that

$$
\int_{a}^{b} d x \Psi(z, x)^{*} A(x) \Psi(z, x)>0
$$

for every interval $(a, b) \subset \mathbb{R}, a<b$.

A principal example of such a system is the Dirac-type system obtained when

$$
A(x)=I_{2 m},
$$


which is the subject of the present paper; another example being the matrix-valued Schrödinger system, obtained when

$$
A(x)=\left(\begin{array}{cc}
I_{m} & 0 \\
0 & 0
\end{array}\right), \quad B(x)=\left(\begin{array}{cc}
-Q(x) & 0 \\
0 & I_{m}
\end{array}\right)
$$

which is the subject of [20. When (2.5) holds, we note that (2.2a) is equivalent to

$$
\begin{aligned}
-\psi_{1}^{\prime \prime}(z, x)+Q(x) \psi_{1}(z, x) & =z \psi_{1}(z, x), \\
\psi_{2}(z, x) & =\psi_{1}^{\prime}(z, x)
\end{aligned}
$$

for a.e. $x \in \mathbb{R}$. Hypothesis 2.2 clearly holds in both examples.

Next, we introduce a set of matrices that will serve as boundary data for separated boundary conditions.

Hypothesis 2.3. Let $\gamma=\left(\gamma_{1} \gamma_{2}\right)$ with $\gamma_{j} \in \mathbb{C}^{m \times m}, j=1,2$. We assume that $\gamma$ satisfies the following conditions:

$$
\operatorname{rank}(\gamma)=m
$$

and either

$$
\operatorname{Im}\left(\gamma_{2} \gamma_{1}^{*}\right) \leq 0, \quad \text { or } \quad \operatorname{Im}\left(\gamma_{2} \gamma_{1}^{*}\right) \geq 0,
$$

where $(2 i)^{-1} \gamma J \gamma^{*}=\operatorname{Im}\left(\gamma_{2} \gamma_{1}^{*}\right)$. Given the rank condition in (2.8a), we assume, without loss of generality in what follows, the normalization

$$
\gamma \gamma^{*}=I_{m}
$$

Remark 2.4. With $\alpha \in \mathbb{C}^{m \times 2 m}$, the conditions

$$
\alpha \alpha^{*}=I_{m}, \quad \alpha J \alpha^{*}=0
$$

imply that $\alpha$ satisfies Hypothesis 2.3 and they explicitly read

$$
\alpha_{1} \alpha_{1}^{*}+\alpha_{2} \alpha_{2}^{*}=I_{m}, \quad \alpha_{2} \alpha_{1}^{*}-\alpha_{1} \alpha_{2}^{*}=0 .
$$

In fact, one also has

$$
\alpha_{1}^{*} \alpha_{1}+\alpha_{2}^{*} \alpha_{2}=I_{m}, \quad \alpha_{2}^{*} \alpha_{1}-\alpha_{1}^{*} \alpha_{2}=0,
$$

as is clear from

$$
\left(\begin{array}{cc}
\alpha_{1} & \alpha_{2} \\
-\alpha_{2} & \alpha_{1}
\end{array}\right)\left(\begin{array}{cc}
\alpha_{1}^{*} & -\alpha_{2}^{*} \\
\alpha_{2}^{*} & \alpha_{1}^{*}
\end{array}\right)=I_{2 m}=\left(\begin{array}{cc}
\alpha_{1}^{*} & -\alpha_{2}^{*} \\
\alpha_{2}^{*} & \alpha_{1}^{*}
\end{array}\right)\left(\begin{array}{cc}
\alpha_{1} & \alpha_{2} \\
-\alpha_{2} & \alpha_{1}
\end{array}\right),
$$

since any left inverse matrix is also a right inverse, and vice versa. Moreover, from (2.11) we obtain

$$
\alpha^{*} \alpha J+J \alpha^{*} \alpha=J
$$

With $\alpha \in \mathbb{C}^{m \times 2 m}$ satisfying (2.9), let $\Psi\left(z, x, x_{0}, \alpha\right)$ be a normalized fundamental system of solutions of (2.2) at some $x_{0} \in \mathbb{R}$. That is, $\Psi\left(z, x, x_{0}, \alpha\right)$ satisfies (2.2) for a.e. $x \in \mathbb{R}$, and

$$
\Psi\left(z, x_{0}, x_{0}, \alpha\right)=\left(\alpha^{*} J \alpha^{*}\right)=\left(\begin{array}{cc}
\alpha_{1}^{*} & -\alpha_{2}^{*} \\
\alpha_{2}^{*} & \alpha_{1}^{*}
\end{array}\right) .
$$

We partition $\Psi\left(z, x, x_{0}, \alpha\right)$ as follows:

$$
\begin{aligned}
\Psi\left(z, x, x_{0}, \alpha\right) & =\left(\Theta\left(z, x, x_{0}, \alpha\right) \Phi\left(z, x, x_{0}, \alpha\right)\right) \\
& =\left(\begin{array}{ll}
\theta_{1}\left(z, x, x_{0}, \alpha\right) & \phi_{1}\left(z, x, x_{0}, \alpha\right) \\
\theta_{2}\left(z, x, x_{0}, \alpha\right) & \phi_{2}\left(z, x, x_{0}, \alpha\right)
\end{array}\right),
\end{aligned}
$$


where $\theta_{j}\left(z, x, x_{0}, \alpha\right)$ and $\phi_{j}\left(z, x, x_{0}, \alpha\right)$ for $j=1,2$ are $m \times m$ matrices, entire with respect to $z \in \mathbb{C}$, and normalized according to (2.14a). One can now prove the following result.

Lemma 2.5. Let $\Theta\left(z, x, x_{0}, \alpha\right)$ and $\Phi\left(z, x, x_{0}, \alpha\right)$ be defined in 2.14b with $\alpha$ and $\beta$ satisfying Hypothesis 2.3 and with $\operatorname{Im}\left(\alpha_{2} \alpha_{1}^{*}\right)=0$. Then, for $c \neq x_{0}, \beta \Phi\left(z, c, x_{0}, \alpha\right)$ is singular if and only if $z$ is an eigenvalue for the regular boundary value problem given by 2.2a on $\left[x_{0}, c\right]$ if $c>x_{0}$ and on $\left[c, x_{0}\right]$ if $c<x_{0}$ together with the separated boundary conditions

$$
\alpha \Psi\left(z, x_{0}\right)=0, \quad \beta \Psi(z, c)=0,
$$

where $\Psi(z, x)=\left(\psi_{1}(z, x)^{t} \psi_{2}(z, x)^{t}\right)^{t}$ with $\psi_{j}(z, \cdot) \in A C\left(\left[x_{0}, c\right]\right)$ if $c>x_{0}$ and $\psi_{j}(z, \cdot) \in A C\left(\left[c, x_{0}\right]\right)$ if $c<x_{0}, j=1,2$.

Note that the regular boundary value problem described in Lemma 2.5 is selfadjoint when $\operatorname{Im}\left(\beta_{2} \beta_{1}^{*}\right)=0$.

In light of Lemma 2.5 it is possible to introduce, under appropriate conditions, the $m \times m$ matrix-valued function $M\left(z, c, x_{0}, \alpha, \beta\right)$, as follows.

Definition 2.6. Let $\Theta\left(z, x, x_{0}, \alpha\right)$ and $\Phi\left(z, x, x_{0}, \alpha\right)$ be defined in (2.14b) with $\alpha$ and $\beta$ satisfying Hypothesis 2.3 and with $\operatorname{Im}\left(\alpha_{2} \alpha_{1}^{*}\right)=0$. For $c \neq x_{0}$, and $\beta \Phi\left(z, c, x_{0}, \alpha\right)$ nonsingular, let

$$
M\left(z, c, x_{0}, \alpha, \beta\right)=-\left[\beta \Phi\left(z, c, x_{0}, \alpha\right)\right]^{-1}\left[\beta \Theta\left(z, c, x_{0}, \alpha\right)\right] .
$$

$M\left(z, c, x_{0}, \alpha, \beta\right)$ is said to be the Weyl-Titchmarsh $M$-function for the regular boundary value problem described in Lemma 2.5.

The Weyl-Titchmarsh $M$-function is an $m \times m$ matrix-valued function with meromorphic entries whose poles correspond to eigenvalues for the regular boundary value problem given by (2.2a) and (2.15). Moreover, if $M \in \mathbb{C}^{m \times m}$, and one defines

$$
U\left(z, x, x_{0}, \alpha\right)=\left(\begin{array}{c}
u_{1}\left(z, x, x_{0}, \alpha\right) \\
u_{2}\left(z, x, x_{0}, \alpha\right)
\end{array}\right)=\Psi\left(z, x, x_{0}, \alpha\right)\left(\begin{array}{c}
I_{m} \\
M
\end{array}\right),
$$

with $u_{j}\left(z, x, x_{0}, \alpha\right) \in \mathbb{C}^{m \times m}, j=1,2$, then $U\left(z, x, x_{0}, \alpha\right)$ will satisfy the boundary condition at $x=c$ in 2.15) whenever $M=M\left(z, c, x_{0}, \alpha, \beta\right)$. Intimately connected with the matrices introduced in Definition 2.6 is the set of $m \times m$ complex matrices known as the Weyl disk. Several characterizations of this set have appeared in the literature (see, e.g., 8], [11, [12, 68], 63], 80, 100]). We now mention two, and will introduce two others in Section 3 which we use in the derivation of the asymptotic expansions that are the subject of Sections 3 and 4

To describe this set, we first introduce the matrix-valued function $E_{c}(M)$ : With $c \neq x_{0}, z \in \mathbb{C} \backslash \mathbb{R}$, and with $U\left(z, c, x_{0}, \alpha\right)$ defined by (2.17) in terms of a matrix $M \in \mathbb{C}^{m \times m}$, let

$$
E_{c}(M)=\sigma\left(x_{0}, c, z\right) U\left(z, c, x_{0}, \alpha\right)^{*}(i J) U\left(z, c, x_{0}, \alpha\right),
$$

where

$$
\sigma(s, t, z)=\frac{(s-t) \operatorname{Im}(z)}{|(s-t) \operatorname{Im}(z)|}
$$

with $s \neq t$, and $s, t \in \mathbb{R}$. For subsequent use we also introduce

$$
\sigma(s, t)=\sigma(s, t, i), \quad \sigma(z)=\sigma(1,0, z),
$$

again assuming $s \neq t, s, t \in \mathbb{R}$. 
Definition 2.7. Let the following be fixed: Real numbers $x_{0}$ and $c \neq x_{0}$, an $m \times 2 m$ matrix $\alpha$ satisfying (2.9), and $z \in \mathbb{C} \backslash \mathbb{R}$. $\mathcal{D}\left(z, c, x_{0}, \alpha\right)$ will denote the collection of all $M \in \mathbb{C}^{m \times m}$ for which $E_{c}(M) \leq 0$, where $E_{c}(M)$ is defined in (2.18). $\mathcal{D}\left(z, c, x_{0}, \alpha\right)$ is said to be a Weyl disk. The set of $M \in \mathbb{C}^{m \times m}$ for which $E_{c}(M)=0$ is said to be a Weyl circle (even when $m>1$ ).

This definition leads to a presentation that is a generalization of the description first given by Weyl [120]; a presentation which is geometric in nature, involves the contractive matrices $V \in \mathbb{C}^{m \times m}$, such that $V V^{*} \leq I_{m}$, and provides the justification for the geometric terms of circle and disk (cf., e.g., [63], 68, [80], [100]).

The disk has also been characterized in terms of matrices which statisfy Hypothesis 2.3 and which serve as boundary data for the regular boundary value problem described in Lemma 2.5 (cf., e.g., [11], [12]). More precisely, one could have used the following alternative definition.

Definition 2.7A. $\mathcal{D}\left(z, c, x_{0}, \alpha\right)$ denotes the collection of all $M \in \mathbb{C}^{m \times m}$ obtained by the construction given in (2.16) where $c \neq x_{0}, z \in \mathbb{C} / \mathbb{R}$, where $\alpha$ and $\beta$ are the $m \times 2 m$ matrices defined in Hypothesis 2.3 for which $\sigma\left(c, x_{0}, z\right) \operatorname{Im}\left(\beta_{2} \beta_{1}^{*}\right) \geq 0$, and $\operatorname{Im}\left(\alpha_{2} \alpha_{1}^{*}\right)=0$.

However, in this paper we take Definition 2.7 as our point of departure.

We note that the Weyl circle corresponds to the regular boundary value problems in Lemma 2.5 with separated, self-adjoint boundary conditions. For convenience of the reader, and to achieve a reasonable level of completeness, we reproduce the corresponding short proof below.

Lemma 2.8 (66], 68, [80]). Let $M \in \mathbb{C}^{m \times m}, c \neq x_{0}$, and $z \in \mathbb{C} \backslash \mathbb{R}$. Then, $E_{c}(M)=0$ if and only if there is a $\beta \in \mathbb{C}^{m \times 2 m}$ satisfying (2.9) such that

$$
0=\beta U\left(z, c, x_{0}, \alpha\right),
$$

where $U(z, c)=U\left(z, c, x_{0}, \alpha\right)$ is defined in (2.17) in terms of $M$. With $\beta$ so defined,

$$
M=-\left[\beta \Phi\left(z, c, x_{0}, \alpha\right)\right]^{-1}\left[\beta \Theta\left(z, c, x_{0}, \alpha\right)\right],
$$

that is, $M=M\left(z, c, x_{0}, \alpha, \beta\right)$. Moreover, $\beta$ may be chosen to satisfy (2.8c), and hence Hypothesis 2.3 .

Proof. Let $z \in \mathbb{C} \backslash \mathbb{R}$, and suppose for a given $M \in \mathbb{C}^{m \times m}$ that there is a $\beta \in$ $\mathbb{C}^{m \times 2 m}$ which satisfies (2.9) and such that (2.21) is satisfied. Given that $\beta J \beta^{*}=$ $2 i \operatorname{Im}\left(\beta_{2} \beta_{1}^{*}\right)=0$, and given that $\operatorname{rank}(\beta)=\operatorname{rank}\left(J \beta^{*}\right)=m$, there is a nonsingular $C \in \mathbb{C}^{m \times m}$ such that $U(z, c)=J \beta^{*} C$. Hence, $E_{c}(M)=i \sigma\left(c, x_{0}, z\right) C^{*} \beta J \beta^{*} C=0$.

Upon showing that $\beta \Phi(z, c)=\beta \Phi\left(z, c, x_{0}, \alpha\right)$ is nonsingular, (2.22) will then follow from (2.21). If $\beta \Phi(z, c)$ is singular, then there are nonzero vectors $v, w \in \mathbb{C}^{m}$ such that $\beta \Phi(z, c) v=0$, and such that $\Phi(z, c) v=J \beta^{*} w$. Let $\Psi_{j}=\Psi_{j}(z, x)$, $j=1,2$, denote solutions of (2.2a) with $z=z_{j}, j=1,2$. Then,

$$
\left(\Psi_{1}^{*} J \Psi_{2}\right)^{\prime}=\left(z_{2}-\bar{z}_{1}\right) \Psi_{1}^{*} A \Psi_{2} .
$$

Using (2.23), and recalling that $\Phi(z, x)$ is defined in (2.14), we obtain

$$
\begin{aligned}
2 i \operatorname{Im}(z) \int_{x_{0}}^{c} d x v^{*} \Phi(z, x)^{*} A(x) \Phi(z, x) v & =v^{*} \Phi(z, c)^{*} J \Phi(z, c) v \\
& =w^{*} \beta J \beta^{*} w=0 .
\end{aligned}
$$

Thus, by Hypothesis $2.2 \operatorname{Im}(z)=0$. This contradicts the assumption that $z \in \mathbb{C} \backslash \mathbb{R}$. 
Conversely, if $E_{c}(M)=0$ for a given $M \in \mathbb{C}^{m \times m}$, then for $z \in \mathbb{C} \backslash \mathbb{R}$ let $\beta=$

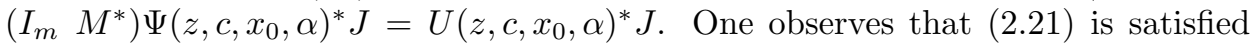
and that $\operatorname{rank}(\beta)=m$. Moreover, $0=\sigma\left(x_{0}, c, z\right) E_{c}(M) / 2=\operatorname{Im}\left(\beta_{2} \beta_{1}^{*}\right)$. If for this choice of $\beta$, (2.8c) is not yet satisfied, one introduces $\Delta=\left(\beta \beta^{*}\right)^{-1 / 2} \beta$ and observes that $0=\Delta U\left(z, c, x_{0}, \alpha\right), \operatorname{Im}\left(\Delta_{2} \Delta_{1}^{*}\right)=\left(\beta \beta^{*}\right)^{-1 / 2} \operatorname{Im}\left(\beta_{2} \beta_{1}^{*}\right)\left(\beta \beta^{*}\right)^{-1 / 2}$, and that $\Delta$ satisfies all requirements of (2.9).

Next, we recall a fundamental property associated with matrices in $\mathcal{D}\left(z, c, x_{0}, \alpha\right)$.

Lemma 2.9. If $M \in \mathcal{D}\left(z, c, x_{0}, \alpha\right)$, then

$$
\sigma\left(c, x_{0}, z\right) \operatorname{Im}(M)>0 .
$$

Moreover, whenever $\beta \in \mathbb{C}^{m \times 2 m}$ satisfies (2.9),

$$
M\left(\bar{z}, c, x_{0}, \alpha, \beta\right)=M\left(z, c, x_{0}, \alpha, \beta\right)^{*} .
$$

Proof. Let $\Psi_{j}=\Psi_{j}(z, x), j=1,2$, denote solutions of (2.2a) with $z=z_{j}, j=1,2$. Then $\left(\Psi_{1}^{*} J \Psi_{2}\right)^{\prime}=\left(z_{2}-\bar{z}_{1}\right) \Psi_{1}^{*} A \Psi_{2}$ as in (2.23). This implies

$$
\begin{aligned}
2 i \operatorname{Im}(z) \int_{x_{0}}^{c} d x U(z, x)^{*} A(x) U(z, x) & =\left.U(z, x)^{*} J U(z, x)\right|_{x_{0}} ^{c} \\
& =2 i \operatorname{Im}(M)+U(z, c)^{*} J U(z, c),
\end{aligned}
$$

with $U(z, x)=U\left(z, x, x_{0}, \alpha\right)$ defined in (2.17). Moreover, by the definition of $E_{c}(M)$ given in (2.18), one obtains

$$
\begin{aligned}
& 2 \sigma\left(c, x_{0}, z\right) \operatorname{Im}(M) \\
& =-E_{c}(M)+2 \sigma\left(c, x_{0}\right)|\operatorname{Im}(z)| \int_{x_{0}}^{c} d s U(z, s)^{*} A(s) U(z, s) .
\end{aligned}
$$

By Hypothesis 2.2 and Definition 2.7 one infers that $\sigma\left(c, x_{0}, z\right) \operatorname{Im}(M)>0$. To prove (2.26) , let $\Psi(z, x)=\Psi\left(z, x, x_{0}, \alpha\right)$, where $\Psi$ is defined in (2.2). Then, by (2.23),

$$
\Psi(\bar{z}, x)^{*} J \Psi(z, x)=J
$$

which implies $J \Psi(z \cdot x)(\Psi(\bar{z}, x) J)^{*}=I_{2 m}$ and hence

$$
\Psi(z, x) J \Psi(\bar{z}, x)^{*}=J .
$$

Thus one concludes that

$$
\beta \Phi(z, c)(\beta \Theta(\bar{z}, c))^{*}-\beta \Theta(z, c)(\beta \Phi(\bar{z}, c))^{*}=\beta J \beta^{*}=0,
$$

from which (2.26) follows immediately by Lemma 2.8 ,

For $c>x_{0}$, the function $M\left(z, c, x_{0}, \alpha, \beta\right)$, defined by (2.16), and satisfying (2.25), is said to be a matrix-valued Herglotz function of rank $m$. Hence, for $\operatorname{Im}\left(\beta_{2} \beta_{1}^{*}\right)=0$, poles of $M\left(z, c, x_{0}, \alpha, \beta\right), c>x_{0}$, are at most of first order, are real, and have nonpositive residues. Such functions admit a representation of the form

$$
\begin{aligned}
M\left(z, c, x_{0}, \alpha, \beta\right)= & C_{1}\left(c, x_{0}, \alpha, \beta\right)+z C_{2}\left(c, x_{0}, \alpha, \beta\right) \\
& +\int_{-\infty}^{\infty} d \Omega\left(\lambda, c, x_{0}, \alpha, \beta\right)\left(\frac{1}{\lambda-z}-\frac{\lambda}{1+\lambda^{2}}\right), \quad c>x_{0},
\end{aligned}
$$


where $C_{2}\left(c, x_{0}, \alpha, \beta\right) \geq 0$ and $C_{1}\left(c, x_{0}, \alpha, \beta\right)$ are self-adjoint $m \times m$ matrices, and where $\Omega\left(\lambda, c, x_{0}, \alpha, \beta\right)$ is a nondecreasing $m \times m$ matrix-valued function such that

$$
\int_{-\infty}^{\infty}\left\|d \Omega\left(\lambda, c, x_{0}, \alpha, \beta\right)\right\|_{\mathbb{C}^{m \times m}}\left(1+\lambda^{2}\right)^{-1}<\infty,
$$

$$
\Omega\left((\lambda, \mu], c, x_{0}, \alpha, \beta\right)=\lim _{\delta \downarrow 0} \lim _{\epsilon \downarrow 0} \frac{1}{\pi} \int_{\lambda+\delta}^{\mu+\delta} d \nu \sigma\left(c, x_{0}\right) \operatorname{Im}\left(M\left(\nu+i \epsilon, c, x_{0}, \alpha, \beta\right)\right) .
$$

In general, for self-adjoint boundary value problems, $\Omega\left(\lambda, c, x_{0}, \alpha, \beta\right)$ is piecewise constant with jump discontinuities precisely at the eigenvalues of the boundary value problem. Moreover, in the matrix-valued Schrödinger and Dirac-type cases, $C_{2}=0$ in (2.32) (and later in (2.64) and (2.79) ). Analogous statements apply to $-M\left(z, c, x_{0}, \alpha, \beta\right)$ if $c<x_{0}$. For such problems, we note in the subsequent lemma that for fixed $\beta$, varying the boundary data $\alpha$ produces Weyl-Titchmarsh matrices $M\left(z, c, x_{0}, \alpha, \beta\right)$ related to each other via linear fractional transformations (see also [47], 52] for a general approach to such linear fractional transformations).

Lemma 2.10. Suppose $\alpha, \beta, \gamma \in \mathbb{C}^{m \times 2 m}$ satisfy (2.9). Let $M_{\alpha}=M\left(z, c, x_{0}, \alpha, \beta\right)$ and $M_{\gamma}=M\left(z, c, x_{0}, \gamma, \beta\right)$. Then,

$$
M_{\alpha}=\left[-\alpha J \gamma^{*}+\alpha \gamma^{*} M_{\gamma}\right]\left[\alpha \gamma^{*}+\alpha J \gamma^{*} M_{\gamma}\right]^{-1} .
$$

Proof. Let $U_{\alpha}(z, x)=U\left(z, x, x_{0}, \alpha\right)$ and $U_{\gamma}(z, x)=U\left(z, x, x_{0}, \gamma\right)$ be defined in (2.17) with $M=M_{\alpha}$ and $M=M_{\gamma}$ respectively. Then,

$$
0=\beta U_{\alpha}(z, c)=\gamma U_{\gamma}(z, c) .
$$

By the rank condition (2.8a),

$$
U_{\alpha}(z, c)=J \beta^{*} C_{\alpha}, \quad U_{\gamma}(z, c)=J \beta^{*} C_{\gamma}
$$

for nonsingular $C_{\alpha}, C_{\gamma} \in \mathbb{C}^{m \times m}$. Thus, by (2.14a), and by the uniqueness of a solution of (2.2a), there is a nonsingular $C \in \mathbb{C}^{m \times m}$ for which

$$
\left(\begin{array}{ll}
\alpha^{*} & J \alpha^{*}
\end{array}\right)\left(\begin{array}{l}
I_{m} \\
M_{\alpha}
\end{array}\right)=U_{\alpha}\left(z, x_{0}\right)=U_{\gamma}\left(z, x_{0}\right) C=\left(\begin{array}{ll}
\gamma^{*} & J \gamma^{*}
\end{array}\right)\left(\begin{array}{l}
I_{m} \\
M_{\gamma}
\end{array}\right) C .
$$

By (2.13),

$$
\left(\begin{array}{cc}
\alpha^{*} & J \alpha^{*}
\end{array}\right)^{-1}=\left(\begin{array}{c}
\alpha \\
-\alpha J
\end{array}\right)
$$

and hence, by (2.37) we see that

$$
\begin{aligned}
I_{m} & =\left(\alpha \gamma^{*}+\alpha J \gamma^{*} M_{\gamma}\right) C, \\
M_{\alpha} & =\left(-\alpha J \gamma^{*}+\alpha \gamma^{*} M_{\gamma}\right) C,
\end{aligned}
$$

from which (2.34) immediately follows.

Remark 2.11. From the proof of the previous lemma one infers, in general, that

$$
U_{\gamma}(z, x)=U_{\alpha}(z, x)\left(\alpha \gamma^{*}+\alpha J \gamma^{*} M_{\gamma}\right) .
$$

Moreover, if $\alpha_{0}=\left(I_{m} 0\right)$ and $\gamma_{0}=\left(\begin{array}{ll}0 & I_{m}\end{array}\right)$, one observes, in particular,

$$
M\left(z, c, x_{0}, \alpha_{0}, \beta\right)=-M\left(z, c, x_{0}, \gamma_{0}, \beta\right)^{-1} .
$$


We further note that the sets $\mathcal{D}\left(z, c, x_{0}, \alpha\right)$ are closed and convex (cf., e.g., 66, [68], 80], [100]). Moreover, by (2.28) and Hypothesis [2.2, one concludes that $E_{c}(M)$ is strictly increasing. This fact together with Lemma 2.8 implies that, as a function of $c$, the sets $\mathcal{D}\left(z, c, x_{0}, \alpha\right)$ are strictly nesting in the sense that

$$
\mathcal{D}\left(z, c_{2}, x_{0}, \alpha\right) \subset \mathcal{D}\left(z, c_{1}, x_{0}, \alpha\right) \quad \text { for } \quad x_{0}<c_{1}<c_{2} \quad \text { or } \quad c_{2}<c_{1}<x_{0} .
$$

Hence, the intersection of this nested sequence, as $c \rightarrow \pm \infty$, is nonempty, closed and convex. We say that this intersection is a limiting set for the nested sequence.

Definition 2.12. Let $\mathcal{D}_{ \pm}\left(z, x_{0}, \alpha\right)$ denote the closed, convex set in the space of $m \times m$ matrices which is the limit, as $c \rightarrow \pm \infty$, of the nested collection of sets $\mathcal{D}\left(z, c, x_{0}, \alpha\right)$ given in Definition [2.7. $\mathcal{D}_{ \pm}\left(z, x_{0}, \alpha\right)$ is said to be a limiting disk. Elements of $\mathcal{D}_{ \pm}\left(z, x_{0}, \alpha\right)$ are denoted by $M_{ \pm}\left(z, x_{0}, \alpha\right) \in \mathbb{C}^{m \times m}$.

In light of the containment described in (2.42), for $c \neq x_{0}$ and $z \in \mathbb{C} \backslash \mathbb{R}$,

$$
\mathcal{D}_{ \pm}\left(z, x_{0}, \alpha\right) \subset \mathcal{D}\left(z, c, x_{0}, \alpha\right),
$$

with emphasis on strict containment of the disks in (2.43). Moreover, by (2.28),

$$
M \in \mathcal{D}_{ \pm}\left(z, x_{0}, \alpha\right) \text { precisely when } E_{c}(M)<0 \text { for all } c \in\left(x_{0}, \pm \infty\right) .
$$

The following lemma appears to have gone unnoted in the literature.

Lemma 2.13. Let $M \in \mathbb{C}^{m \times m}, c \neq x_{0}$, and $z \in \mathbb{C} \backslash \mathbb{R}$. Then, $E_{c}(M)<0$ if and only if there is a $\beta \in \mathbb{C}^{m \times 2 m}$ satisfying the condition

$$
\sigma\left(c, x_{0}, z\right) \operatorname{Im}\left(\beta_{2} \beta_{1}^{*}\right)>0,
$$

and such that (2.21) holds with $u_{j}(z, c)=u_{j}\left(z, c, x_{0}, \alpha\right), j=1,2$, defined in (2.17) in terms of $M$. With $\beta$ so defined, (2.22) holds; that is, $M=M\left(z, c, x_{0}, \alpha, \beta\right)$. Moreover, $\beta$ maybe chosen to satisfy (2.8c), and hence Hypothesis 2.3.

Proof. Let $z \in \mathbb{C} \backslash \mathbb{R}$, and for a given $M \in \mathbb{C}^{m \times m}$ suppose that there is a $\beta \in \mathbb{C}^{m \times 2 m}$ satisfying (2.45) such that (2.21) holds. The matrices $\beta_{j}, j=1,2$, are invertible by (2.45), and by (2.21) it follows that

$$
U(z, c)=\left(\begin{array}{c}
-\beta_{1}^{-1} \beta_{2} \\
I_{m}
\end{array}\right) u_{2}(z, c) .
$$

By (2.18) and (2.46), one then concludes that

$$
E_{c}(M)=-2 \sigma\left(c, x_{0}, z\right) u_{2}(z, c)^{*} \beta_{1}^{-1} \operatorname{Im}\left(\beta_{2} \beta_{1}^{*}\right)\left(\beta_{1}^{*}\right)^{-1} u_{2}(z, c),
$$

and hence that $E_{c}(M)<0$ whenever (2.45) holds.

Upon showing that $\beta \Phi(z, c)$ is nonsingular, (2.22) will follow from (2.21). If $\beta \Phi(z, c)$ is singular, then there is a nonzero vector $v \in \mathbb{C}^{m}$ such that $\beta \Phi(z, c) v=0$. By the nonsingularity of $\beta_{j}, j=1,2, \phi_{1}(z, c) v=-\beta_{1}^{-1} \beta_{2} \phi_{2}(z, c) v$, and as a result, (2.24a) yields

$$
\begin{aligned}
& 2 \sigma\left(c, x_{0}\right)|\operatorname{Im}(z)| \int_{x_{0}}^{c} d x v^{*} \Phi(z, x)^{*} A(x) \Phi(z, x) v \\
& =-2 \sigma\left(c, x_{0}, z\right) v^{*} \phi_{2}(z, c)^{*} \beta_{1}^{-1} \operatorname{Im}\left(\beta_{2} \beta_{1}^{*}\right)\left(\beta_{1}^{*}\right)^{-1} \phi_{2}(z, c) v,
\end{aligned}
$$

and hence, a contradiction to (2.45) (cf. (2.3)) .

Conversely, if $E_{c}(M)<0$ for a given $M \in \mathbb{C}^{m \times m}$, then for $z \in \mathbb{C} \backslash \mathbb{R}, u_{j}(z, c)$, $j=1,2$, defined by (2.17), are nonsingular. Indeed, if either $u_{1}(z, c)$ or $u_{2}(z, c)$ is singular, then there is a $v \in \mathbb{C}^{m}, v \neq 0$, such that $v^{*} E_{c}(M) v=0$, a contradiction. 
Next, let $\beta_{1}=I_{m}$ and let $\beta_{2}=-u_{1}(z, c) u_{2}(z, c)^{-1}$. Then, for these $\beta_{j}, j=1,2$, (2.21) holds. Equation (2.47) now implies that $\sigma\left(c, x_{0}, z\right) \operatorname{Im}\left(\beta_{2} \beta_{1}^{*}\right)>0$ for $c \neq x_{0}$ and $z \in \mathbb{C} \backslash \mathbb{R}$. For this choice, $\beta$ does not satisfy (2.8c). However, one can normalize $\beta$ as described in the proof of Lemma 2.8 .

Hence by Lemma 2.13 and (2.44), we see that if $M \in \mathcal{D}_{ \pm}\left(z, x_{0}, \alpha\right)$, then for some $\beta \in \mathbb{C}^{m \times 2 m}$ satisfying (2.45),

$$
M_{ \pm}\left(z, x_{0}, \alpha\right)=M\left(z, c, x_{0}, \alpha, \beta\right) .
$$

Remark 2.14. To the reader of [20, our study of the high-energy asymptotics of the Weyl-Titchmarsh $M$-function for matrix Schrödinger operators, we offer this cautionary note: In 20], $D\left(z, c, x_{0}, \alpha\right)$ represents the set of matrices characterized by Lemmas 2.8 and 2.13 However, the homeomorphism that exists between the contractive matrices $V \in \mathbb{C}^{m \times m}, V V^{*} \leq I_{m}$, and the Weyl disk $D\left(z, c, x_{0}, \alpha\right)$ (cf., 66], 68], 80, [100]) shows that those $M \in \mathbb{C}^{m \times m}$ characterized in Lemma 2.8 correspond to the set of unitary matrices while those characterized in Lemma 2.13 correspond to the contractive matrices for which $V V^{*}<I_{m}$. Hence, Lemma 2.8 characterizes part of the boundary while Lemma 2.13 characterizes the interior of the Weyl disk as it is defined in Defintion 2.7. As a result, the closure of the set consisting of those $M \in \mathbb{C}^{m \times m}$ characterized by these two lemmas (i.e., those $M$ which correspond to $V V^{*}<I_{m}$, or to $\left.V V^{*}=I_{m}\right)$ is the Weyl disk. Thus, for deriving high-energy asymptotics for $M_{ \pm}\left(z, x_{0}, \alpha\right)$, it is sufficient to consider the subset of the Weyl disk consisting of those matrices $M \in \mathbb{C}^{m \times m}$ characterized in Lemma 2.8 and Lemma 2.13. This was the approach taken in [20].

When $\mathcal{D}_{ \pm}\left(z, x_{0}, \alpha\right)$ is a singleton matrix, the system $\left.2.2 \mathrm{a}\right)$ is said to be in the limit point (l.p.) case at $\pm \infty$. If $\mathcal{D}_{ \pm}\left(z, x_{0}, \alpha\right)$ has nonempty interior, then (2.2a) is said to be in the limit circle (l.c.) case at $\pm \infty$. Indeed, for the case $m=1$, the limit point case corresponds to a point in $\mathbb{C}$, whereas the limit circle case corresponds to $\mathcal{D}_{ \pm}\left(z, x_{0}, \alpha\right)$ being a disk in $\mathbb{C}$.

These apparent geometric properties for the disk correspond to analytic properties for the solutions of the Hamiltonian system (2.2a). To recall this correspondence, we introduce the following spaces, in which we assume that $-\infty \leq a<b \leq$ $\infty$ :

$$
\begin{aligned}
L_{A}^{2}((a, b)) & =\left\{\phi:(a, b) \rightarrow \mathbb{C}^{2 m} \mid \int_{a}^{b} d x(\phi(x), A \phi(x))_{\mathbb{C}^{2 m}}<\infty\right\}, \\
N(z, \infty) & =\left\{\phi \in L_{A}^{2}((c, \infty)) \mid J \phi^{\prime}=(z A+B) \phi \text { a.e. on }(c, \infty)\right\}, \\
N(z,-\infty) & =\left\{\phi \in L_{A}^{2}((-\infty, c)) \mid J \phi^{\prime}=(z A+B) \phi \text { a.e. on }(-\infty, c)\right\},
\end{aligned}
$$

for some $c \in \mathbb{R}$ and $z \in \mathbb{C}$. (Here $(\phi, \psi)_{\mathbb{C}^{n}}=\sum_{j=1}^{n} \bar{\phi}_{j} \psi_{j}$ denotes the standard scalar product in $\mathbb{C}^{n}$, abbreviating $\chi \in \mathbb{C}^{n}$ by $\chi=\left(\chi_{1}, \ldots, \chi_{n}\right)^{t}$.) Both dimensions of the spaces in (2.50b) and 2.50c), $\operatorname{dim}_{\mathbb{C}}(N(z, \infty))$ and $\operatorname{dim}_{\mathbb{C}}(N(z,-\infty))$, are constant for $z \in \mathbb{C}_{ \pm}=\{\zeta \in \mathbb{C} \mid \pm \operatorname{Im}(\zeta)>0\}$ (see, e.g., 8], 75]). One then observes that the Hamiltonian system (2.2a) is in the limit point case at $\pm \infty$ whenever

$$
\operatorname{dim}_{\mathbb{C}}(N(z, \pm \infty))=m \text { for all } z \in \mathbb{C} \backslash \mathbb{R}
$$

and in the limit circle case at $\pm \infty$ whenever

$$
\operatorname{dim}_{\mathbb{C}}(N(z, \pm \infty))=2 m \text { for all } z \in \mathbb{C} .
$$


Next we show that the Dirac-type systems considered in this paper are always in the limit point case at $\pm \infty$. Results of this type, under varying sets of assumptions on $B(x)$, are well known to experts in the field. For instance, in the case $m=1$ and with $B_{1,2}(x)=B_{2,1}(x)$, this fact can be found in [119]. For $B \in C(\mathbb{R})^{2 m \times 2 m}$ and a more general constant matrix $A$, this result is proven in 82 (their proof, however, extends to the current $B \in L_{\text {loc }}^{1}(\mathbb{R})$ case). More generally, multi-dimensional Dirac operators with $L_{\text {loc }}^{2}\left(\mathbb{R}^{n}\right)$-type coefficients (and additional conditions) can be found in [84]. A short proof in the case $m=1$ has recently been sent to us by Don Hinton [59]. For convenience of the reader we present its elementary generalization to $m \in$ $\mathbb{N}$ below (see also [19] for a sketch of such a proof). After completion of this paper we became aware of a recent preprint by Lesch and Malamud 83] which provides a thorough study of self-adjointness questions for more general Hamiltonian systems than those studied in this paper.

Lemma 2.15. The limit point case holds for Dirac-type systems (i.e., for $A=I_{2 m}$ in (2.2a ) at $\pm \infty$.

Proof. Let $\left\{y_{\ell}(z, x)\right\}_{\ell=1, \ldots, k}$ and $\left\{w_{j}(z, x)\right\}_{j=1, \ldots, k^{\prime}}$ denote bases for $N(z, \pm \infty)$ and $N(\bar{z}, \pm \infty)$, respectively. By Theorem 9.11.1 of Atkinson [8], one has $k, k^{\prime} \geq m$ for $z \in \mathbb{C} \backslash \mathbb{R}$. We now assume that $k>m$.

One observes that $\left\{y_{1}(z, c), \ldots, y_{k}(z, c)\right\}$ and $\left\{w_{1}(\bar{z}, c), \ldots, w_{k^{\prime}}(\bar{z}, c)\right\}$ are linearly independent in $\mathbb{C}^{2 m}$, where $k+k^{\prime} \geq 2 m+1$. Consequently, there is some $s \in$ $\{1, \ldots, k\}$ and some $r \in\left\{1, \ldots, k^{\prime}\right\}$ such that

$$
w_{r}(\bar{z}, c)^{*} J y_{s}(z, c) \neq 0 \text {. }
$$

By Lagrange's identity,

$$
w_{r}(\bar{z}, x)^{*} J y_{s}(z, x)=w_{r}(\bar{z}, c)^{*} J y_{s}(z, c)
$$

is constant with respect to $x$. On the other hand, an application of Cauchy's inequality shows that the left-hand side of (2.54) is in $L^{1}((c, \pm \infty))$. By 2.53) one obtains a contradiction and hence concludes that

$$
\operatorname{dim}_{\mathbb{C}}(N(z, \pm \infty))=m .
$$

The analogous argument then also yields

$$
\operatorname{dim}_{\mathbb{C}}(N(\bar{z}, \pm \infty))=m
$$

and hence the limit point property of Dirac-type systems with $A(x)=I_{2 m}$ in (2.2a).

Returning to the general case (2.2a), in either the limit point or limit circle cases, $M_{ \pm}\left(z, x_{0}, \alpha\right) \in \partial \mathcal{D}_{ \pm}\left(z, x_{0}, \alpha\right)$ is said to be a half-line Weyl-Titchmarsh matrix. Each such matrix is associated with the construction of a self-adjoint operator acting on $L_{A}^{2}\left(\left[x_{0}, \pm \infty\right)\right) \cap \mathrm{AC}\left(\left[x_{0}, \pm \infty\right)\right)^{2 m}$ for the Hamiltonian system (2.2a) $)$. However, for those intermediate cases where $m<\operatorname{dim}_{\mathbb{C}}(N(z, \pm \infty))<2 m$, Hinton and Schneider have noted that not every element of $\partial \mathcal{D}_{ \pm}\left(z, x_{0}, \alpha\right)$ is a half-line WeylTitchmarsh matrix, and have characterized those elements of the boundary that are (cf. 68, 69]).

For convenience of the reader we next summarize some of the principal results on half-line Weyl-Titchmarsh matrices. 
Theorem 2.16 (3], [17, 52], 63, [64], 67], [79]). Assume Hypotheses [2.1] and 2.2. Let $z \in \mathbb{C} \backslash \mathbb{R}, x_{0} \in \mathbb{R}$, and denote by $\alpha, \gamma \in \mathbb{C}^{m \times 2 m}$ matrices satisfying (2.9). Then:

(i) $\pm M_{ \pm}\left(z, x_{0}, \alpha\right)$ is an $m \times m$ matrix-valued Herglotz function of maximal rank. In particular,

$$
\begin{gathered}
\operatorname{Im}\left( \pm M_{ \pm}\left(z, x_{0}, \alpha\right)\right)>0, \quad z \in \mathbb{C}_{+}, \\
M_{ \pm}\left(\bar{z}, x_{0}, \alpha\right)=M_{ \pm}\left(z, x_{0}, \alpha\right)^{*}, \\
\operatorname{rank}\left(M_{ \pm}\left(z, x_{0}, \alpha\right)\right)=m, \\
\lim _{\varepsilon \downarrow 0} M_{ \pm}\left(\lambda+i \varepsilon, x_{0}, \alpha\right) \text { exists for a.e. } \lambda \in \mathbb{R}, \\
M_{ \pm}\left(z, x_{0}, \alpha\right)=\left[-\alpha J \gamma^{*}+\alpha \gamma^{*} M_{ \pm}\left(z, x_{0}, \gamma\right)\right] \\
\times\left[\alpha \gamma^{*}+\alpha J \gamma^{*} M_{ \pm}\left(z, x_{0}, \gamma\right)\right]^{-1} .
\end{gathered}
$$

Local singularities of $\pm M_{ \pm}\left(z, x_{0}, \alpha\right)$ and $\mp M_{ \pm}\left(z, x_{0}, \alpha\right)^{-1}$ are necessarily real and at most of first order in the sense that

$$
\begin{aligned}
& \mp \lim _{\epsilon \downarrow 0}\left(i \epsilon M_{ \pm}\left(\lambda+i \epsilon, x_{0}, \alpha\right)\right) \geq 0, \quad \lambda \in \mathbb{R}, \\
& \pm \lim _{\epsilon \downarrow 0}\left(\frac{i \epsilon}{M_{ \pm}\left(\lambda+i \epsilon, x_{0}, \alpha\right)}\right) \geq 0, \quad \lambda \in \mathbb{R} .
\end{aligned}
$$

(ii) $\pm M_{ \pm}\left(z, x_{0}, \alpha\right)$ admit the representations

$$
\begin{aligned}
& (2.64) \pm M_{ \pm}\left(z, x_{0}, \alpha\right)=F_{ \pm}\left(x_{0}, \alpha\right)+\int_{\mathbb{R}} d \Omega_{ \pm}\left(\lambda, x_{0}, \alpha\right)\left((\lambda-z)^{-1}-\lambda\left(1+\lambda^{2}\right)^{-1}\right) \\
& (2.65)=\exp \left(C_{ \pm}\left(x_{0}, \alpha\right)+\int_{\mathbb{R}} d \lambda \Xi_{ \pm}\left(\lambda, x_{0}, \alpha\right)\left((\lambda-z)^{-1}-\lambda\left(1+\lambda^{2}\right)^{-1}\right)\right)
\end{aligned}
$$

where

$$
\begin{array}{ll}
F_{ \pm}\left(x_{0}, \alpha\right)=F_{ \pm}\left(x_{0}, \alpha\right)^{*}, & \int_{\mathbb{R}}\left\|d \Omega_{ \pm}\left(\lambda, x_{0}, \alpha\right)\right\|_{\mathbb{C}^{m \times m}}\left(1+\lambda^{2}\right)^{-1}<\infty \\
C_{ \pm}\left(x_{0}, \alpha\right)=C_{ \pm}\left(x_{0}, \alpha\right)^{*}, & 0 \leq \Xi_{ \pm}\left(\cdot, x_{0}, \alpha\right) \leq I_{m} \text { a.e. }
\end{array}
$$

Moreover,

$$
\begin{aligned}
\Omega_{ \pm}\left((\lambda, \mu], x_{0}, \alpha\right) & =\lim _{\delta \downarrow 0} \lim _{\varepsilon \downarrow 0} \frac{1}{\pi} \int_{\lambda+\delta}^{\mu+\delta} d \nu \operatorname{Im}\left( \pm M_{ \pm}\left(\nu+i \varepsilon, x_{0}, \alpha\right)\right), \\
\Xi_{ \pm}\left(\lambda, x_{0}, \alpha\right) & =\lim _{\varepsilon \downarrow 0} \pi^{-1} \operatorname{Im}\left(\ln \left( \pm M_{ \pm}\left(\lambda+i \varepsilon, x_{0}, \alpha\right)\right)\right) \text { for a.e. } \lambda \in \mathbb{R} .
\end{aligned}
$$

(iii) Define the $2 m \times m$ matrices

$$
\begin{aligned}
U_{ \pm}\left(z, x, x_{0}, \alpha\right) & =\left(\begin{array}{l}
u_{ \pm, 1}\left(z, x, x_{0}, \alpha\right) \\
u_{ \pm, 2}\left(z, x, x_{0}, \alpha\right)
\end{array}\right)=\Psi\left(z, x, x_{0}, \alpha\right)\left(\begin{array}{c}
I_{m} \\
M_{ \pm}\left(z, x_{0}, \alpha\right)
\end{array}\right) \\
& =\left(\begin{array}{cc}
\theta_{1}\left(z, x, x_{0}, \alpha\right) & \phi_{1}\left(z, x, x_{0}, \alpha\right) \\
\theta_{2}\left(z, x, x_{0}, \alpha\right) & \phi_{2}\left(z, x, x_{0}, \alpha\right)
\end{array}\right)\left(\begin{array}{c}
I_{m} \\
M_{ \pm}\left(z, x_{0}, \alpha\right)
\end{array}\right),
\end{aligned}
$$

with $\theta_{j}\left(z, x, x_{0}, \alpha\right)$ and $\phi_{j}\left(z, x, x_{0}, \alpha\right), j=1,2$, defined by (2.14c). Then,

$$
\operatorname{Im}\left(M_{ \pm}\left(z, x_{0}, \alpha\right)\right)=\operatorname{Im}(z) \int_{x_{0}}^{ \pm \infty} d s U_{ \pm}\left(z, s, x_{0}, \alpha\right)^{*} A(s) U_{ \pm}\left(z, s, x_{0}, \alpha\right) .
$$


In the Dirac-type context, where $A=I_{2 m}$, the $m$ columns of $U_{ \pm}\left(z, \cdot, x_{0}, \alpha\right)$ span $N(z, \pm \infty)$.

Up to this point, we focused exclusively on Hamiltonian systems, and neglected the notion of a linear operator associated with (2.2). We did this on purpose, as the formalism presented thus far is widely applicable and goes beyond the prime candidates such as Schrödinger and Dirac-type systems. However, in the remainder of this section and for the bulk of the material from Section 3 on, we will focus on the Dirac-type case. Thus, in addition to Hypotheses 2.1 2.3, which are assumed throughout this paper, we introduce the following hypothesis, tailored to these occasions.

Hypothesis 2.17. Assume Hypotheses 2.1 and 2.3 as well as the Dirac-type assumption (2.4).

Assuming the Dirac-type Hypothesis 2.17, we now describe the associated Diractype operator $D$ on $\mathbb{R}$ by first introducing the Green's matrix associated with (2.2) and (2.4). Define the $2 m \times 2 m$ matrix $G$ by

$$
\begin{gathered}
G\left(z, x, x^{\prime}\right)=U_{\mp}\left(z, x, x_{0}, \alpha_{0}\right)\left[M_{-}\left(z, x_{0}, \alpha_{0}\right)-M_{+}\left(z, x_{0}, \alpha_{0}\right)\right]^{-1} U_{ \pm}\left(\bar{z}, x^{\prime}, x_{0}, \alpha_{0}\right)^{*}, \\
(2.72) \quad \alpha_{0}=\left(I_{m} 0\right), \quad x \lessgtr x^{\prime}, z \in \mathbb{C} \backslash \mathbb{R} .
\end{gathered}
$$

Next, let $\phi \in L^{2}(\mathbb{R})^{2 m}$ and consider

$$
J \psi^{\prime}(z, x)=\left(z I_{2 m}+B(x)\right) \psi(z, x)+\phi(x), \quad z \in \mathbb{C} \backslash \mathbb{R},
$$

for a.e. $x \in \mathbb{R}$. Then, as one can infer from 63 and 65 , (2.73) has a unique solution $\psi(z, \cdot) \in L^{2}(\mathbb{R})^{2 m} \cap \mathrm{AC}_{\text {loc }}(\mathbb{R})^{2 m}$ given by

$$
\psi(z, x)=\int_{\mathbb{R}} d x^{\prime} G\left(z, x, x^{\prime}\right) \phi\left(x^{\prime}\right) .
$$

The Dirac-type operator $D$ in $L^{2}(\mathbb{R})^{2 m}$ associated with the Hamiltonian system (2.2) and (2.4) is then defined by

$$
\left((D-z)^{-1} \psi\right)(x)=\int_{\mathbb{R}} d x^{\prime} G\left(z, x, x^{\prime}\right) \psi\left(x^{\prime}\right), \quad \psi \in L^{2}(\mathbb{R})^{2 m}, z \in \mathbb{C} \backslash \mathbb{R} .
$$

Explicitly, one obtains

$$
\begin{aligned}
D & =J \frac{d}{d x}-B, \\
\operatorname{dom}(D) & =\left\{\phi \in L^{2}(\mathbb{R})^{2 m} \mid \phi \in \mathrm{AC}_{\mathrm{loc}}(\mathbb{R})^{2 m} ;\left(J \phi^{\prime}-B \phi\right) \in L^{2}(\mathbb{R})^{2 m}\right\},
\end{aligned}
$$

taking into account the limit point property of Dirac-type systems as described in Lemma 2.15. Thus, $D$ is a self-adjoint operator in $L^{2}(\mathbb{R})^{2 m}$.

As described in 63 67, the $2 m \times 2 m$ Weyl-Titchmarsh matrix $M\left(z, x_{0}, \alpha_{0}\right)$ associated with $D$ is then defined by

$$
\begin{aligned}
M\left(z, x_{0}, \alpha_{0}\right) & =\left(M_{j, j^{\prime}}\left(z, x_{0}, \alpha_{0}\right)\right)_{j, j^{\prime}=1,2} \\
& =\left[G\left(z, x_{0}, x_{0}+0\right)+G\left(z, x_{0}, x_{0}-0\right)\right] / 2, \quad z \in \mathbb{C} \backslash \mathbb{R} .
\end{aligned}
$$


Actually, one can replace $\alpha_{0}=\left(I_{m} 0\right)$ by an arbitrary matrix $\alpha=\left[\begin{array}{ll}\alpha_{1} & \alpha_{2}\end{array}\right] \in \mathbb{C}^{m \times 2 m}$ satisfying (2.9). Hence one introduces

$$
M\left(z, x_{0}, \alpha\right)=\left(M_{j, j^{\prime}}\left(z, x_{0}, \alpha\right)\right)_{j, j^{\prime}=1,2}, \quad z \in \mathbb{C} \backslash \mathbb{R},
$$

$$
\begin{aligned}
& M_{1,1}\left(z, x_{0}, \alpha\right)=\left[M_{-}\left(z, x_{0}, \alpha\right)-M_{+}\left(z, x_{0}, \alpha\right)\right]^{-1}, \\
& M_{1,2}\left(z, x_{0}, \alpha\right)=2^{-1}\left[M_{-}\left(z, x_{0}, \alpha\right)-M_{+}\left(z, x_{0}, \alpha\right)\right]^{-1}\left[M_{-}\left(z, x_{0}, \alpha\right)+M_{+}\left(z, x_{0}, \alpha\right)\right], \\
& M_{2,1}\left(z, x_{0}, \alpha\right)=2^{-1}\left[M_{-}\left(z, x_{0}, \alpha\right)+M_{+}\left(z, x_{0}, \alpha\right)\right]\left[M_{-}\left(z, x_{0}, \alpha\right)-M_{+}\left(z, x_{0}, \alpha\right)\right]^{-1}, \\
& M_{2,2}\left(z, x_{0}, \alpha\right)=M_{ \pm}\left(z, x_{0}, \alpha\right)\left[M_{-}\left(z, x_{0}, \alpha\right)-M_{+}\left(z, x_{0}, \alpha\right)\right]^{-1} M_{\mp}\left(z, x_{0}, \alpha\right) .
\end{aligned}
$$

The basic results on $M\left(z, x_{0}, \alpha\right)$ then read as follows.

Theorem 2.18 (52, 63, 64], 67, 79]). Assume Hypothesis 2.17, and suppose that $z \in \mathbb{C} \backslash \mathbb{R}, x_{0} \in \mathbb{R}$, and that $\alpha \in \mathbb{C}^{m \times 2 m}$ satisfies (2.9). Then:

(i) $M\left(z, x_{0}, \alpha\right)$ is a matrix-valued Herglotz function of rank $2 m$ with representation

$$
\begin{aligned}
& M\left(z, x_{0}, \alpha\right)=F\left(x_{0}, \alpha\right)+\int_{\mathbb{R}} d \Omega\left(\lambda, x_{0}, \alpha\right)\left((\lambda-z)^{-1}-\lambda\left(1+\lambda^{2}\right)^{-1}\right), \\
& =\exp \left(C\left(x_{0}, \alpha\right)+\int_{\mathbb{R}} d \lambda \Upsilon\left(\lambda, x_{0}, \alpha\right)\left((\lambda-z)^{-1}-\lambda\left(1+\lambda^{2}\right)^{-1}\right)\right),
\end{aligned}
$$

where

$$
\begin{array}{ll}
F\left(x_{0}, \alpha\right)=F\left(x_{0}, \alpha\right)^{*}, & \int_{\mathbb{R}}\left\|d \Omega\left(\lambda, x_{0}, \alpha\right)\right\|_{\mathbb{C}^{2 m \times 2 m}}\left(1+\lambda^{2}\right)^{-1}<\infty, \\
C\left(x_{0}, \alpha\right)=C\left(x_{0}, \alpha\right)^{*}, & 0 \leq \Upsilon\left(\cdot, x_{0}, \alpha\right) \leq I_{2 m} \quad \text { a.e. }
\end{array}
$$

Moreover,

$$
\begin{aligned}
\Omega\left((\lambda, \mu], x_{0}, \alpha\right) & =\lim _{\delta \downarrow 0} \lim _{\varepsilon \downarrow 0} \frac{1}{\pi} \int_{\lambda+\delta}^{\mu+\delta} d \nu \operatorname{Im}\left(M\left(\nu+i \varepsilon, x_{0}, \alpha\right)\right), \\
\Upsilon\left(\lambda, x_{0}, \alpha\right) & =\lim _{\varepsilon \downarrow 0} \pi^{-1} \operatorname{Im}\left(\ln \left(M\left(\lambda+i \varepsilon, x_{0}, \alpha\right)\right)\right) \text { for a.e. } \lambda \in \mathbb{R} .
\end{aligned}
$$

(ii) $z \in \mathbb{C} \backslash \operatorname{spec}(D)$ if and only if $M\left(z, x_{0}, \alpha\right)$ is holomorphic near $z$.

Here $\operatorname{spec}(T)$ abbreviates the spectrum of a linear operator $T$.

Next, we explicitly discuss the elementary Dirac-type example where $A=I_{2 m}$ and $B=0$. 
Example 2.19. Suppose $A=I_{2 m}, B=0$, and let $\alpha \in \mathbb{C}^{m \times 2 m}$ satisfy (2.9). Then,

$$
\begin{aligned}
& \Theta\left(z, x, x_{0}, \alpha\right)=\left(\begin{array}{c}
\theta_{1}\left(z, x, x_{0}, \alpha\right) \\
\theta_{2}\left(z, x, x_{0}, \alpha\right)
\end{array}\right)=\left(\begin{array}{c}
\alpha_{1}^{*} \cos \left(z\left(x-x_{0}\right)\right)+\alpha_{2}^{*} \sin \left(z\left(x-x_{0}\right)\right) \\
\alpha_{2}^{*} \cos \left(z\left(x-x_{0}\right)\right)-\alpha_{1}^{*} \sin \left(z\left(x-x_{0}\right)\right)
\end{array}\right), \\
& z \in \mathbb{C} \text {, } \\
& \Phi\left(z, x, x_{0}, \alpha\right)=\left(\begin{array}{c}
\phi_{1}\left(z, x, x_{0}, \alpha\right) \\
\phi_{2}\left(z, x, x_{0}, \alpha\right)
\end{array}\right)=\left(\begin{array}{c}
-\alpha_{2}^{*} \cos \left(z\left(x-x_{0}\right)\right)+\alpha_{1}^{*} \sin \left(z\left(x-x_{0}\right)\right) \\
\alpha_{1}^{*} \cos \left(z\left(x-x_{0}\right)\right)+\alpha_{2}^{*} \sin \left(z\left(x-x_{0}\right)\right)
\end{array}\right), \\
& U_{ \pm}\left(z, x, x_{0}, \alpha\right)=\left(\begin{array}{c}
u_{ \pm, 1}\left(z, x, x_{0}, \alpha\right) \\
u_{ \pm, 2}\left(z, x, x_{0}, \alpha\right)
\end{array}\right)=\left(\begin{array}{c}
\alpha_{1}^{*} \mp i \alpha_{2}^{*} \\
\pm i\left(\alpha_{1}^{*} \mp i \alpha_{2}^{*}\right)
\end{array}\right) \exp \left( \pm i z\left(x-x_{0}\right)\right),
\end{aligned}
$$

$$
M_{ \pm}(z, x, \alpha)= \pm i I_{m}, \quad z \in \mathbb{C}_{+} .
$$

Compared to the case of Schrödinger operators, it is remarkable that $M_{ \pm}(z, x, \alpha)$ in (2.88) is, in fact, independent of $\alpha$. Put differently, in Dirac-type situations, $M_{ \pm}(z, x, \alpha)$ may contain no information on the boundary condition indexed by $\alpha \in \mathbb{C}^{m \times 2 m}$.

In Sections 4 and 5 we will also refer to half-line Dirac operators $D_{+}(\alpha)$ in $L^{2}\left(\left[x_{0}, \infty\right)\right)^{2 m}$ associated with a self-adjoint boundary condition at $x_{0}$ indexed by $\alpha \in \mathbb{C}^{m \times 2 m}$ satisfying (2.9), and hence we briefly introduce

$$
\begin{aligned}
& D_{+}(\alpha)=J \frac{d}{d x}-B, \\
& \operatorname{dom}\left(D_{+}(\alpha)\right)=\left\{\phi \in L^{2}\left(\left[x_{0}, \infty\right)\right)^{2 m} \mid \phi \in \mathrm{AC}\left(\left[x_{0}, R\right]\right)^{2 m} \text { for all } R>0 ;\right. \\
&\left.\alpha \phi\left(x_{0}\right)=0 ;\left(J \phi^{\prime}-B \phi\right) \in L^{2}\left(\left[x_{0}, \infty\right)\right)^{2 m}\right\},
\end{aligned}
$$

taking into account the limit point property of Dirac-type systems at $+\infty$ as described in Lemma 2.15. Thus, $D_{+}(\alpha)$ is a self-adjoint operator in $L^{2}\left(\left[x_{0}, \infty\right)\right)^{2 m}$. In complete analogy one introduces $D_{-}(\alpha)$ in $L^{2}\left(\left(-\infty, x_{0}\right]\right)^{2 m}$.

Next, we recall a few formulas in connection with Lagrange's identity needed in the proof of Theorem 5.3. assuming $\alpha \in \mathbb{C}^{m \times 2 m}$ satisfies (2.9). Then, explicitly, (2.29) and (2.30) read

$$
\begin{aligned}
\theta_{2}\left(\bar{z}, x, x_{0}, \alpha\right)^{*} \theta_{1}\left(z, x, x_{0}, \alpha\right)-\theta_{1}\left(\bar{z}, x, x_{0}, \alpha\right)^{*} \theta_{2}\left(z, x, x_{0}, \alpha\right) & =0, \\
\phi_{2}\left(\bar{z}, x, x_{0}, \alpha\right)^{*} \phi_{1}\left(z, x, x_{0}, \alpha\right)-\phi_{1}\left(\bar{z}, x, x_{0}, \alpha\right)^{*} \phi_{2}\left(z, x, x_{0}, \alpha\right) & =0 \\
\phi_{2}\left(\bar{z}, x, x_{0}, \alpha\right)^{*} \theta_{1}\left(z, x, x_{0}, \alpha\right)-\phi_{1}\left(\bar{z}, x, x_{0}, \alpha\right)^{*} \theta_{2}\left(z, x, x_{0}, \alpha\right) & =I_{m}, \\
\theta_{1}\left(\bar{z}, x, x_{0}, \alpha\right)^{*} \phi_{2}\left(z, x, x_{0}, \alpha\right)-\theta_{2}\left(\bar{z}, x, x_{0}, \alpha\right)^{*} \phi_{1}\left(z, x, x_{0}, \alpha\right) & =I_{m},
\end{aligned}
$$

and

$$
\begin{aligned}
& \phi_{1}\left(z, x, x_{0}, \alpha\right) \theta_{1}\left(\bar{z}, x, x_{0}, \alpha\right)^{*}-\theta_{1}\left(z, x, x_{0}, \alpha\right) \phi_{1}\left(\bar{z}, x, x_{0}, \alpha\right)^{*}=0, \\
& \phi_{2}\left(z, x, x_{0}, \alpha\right) \theta_{2}\left(\bar{z}, x, x_{0}, \alpha\right)^{*}-\theta_{2}\left(z, x, x_{0}, \alpha\right) \phi_{2}\left(\bar{z}, x, x_{0}, \alpha\right)^{*}=0, \\
& \phi_{2}\left(z, x, x_{0}, \alpha\right) \theta_{1}\left(\bar{z}, x, x_{0}, \alpha\right)^{*}-\theta_{2}\left(z, x, x_{0}, \alpha\right) \phi_{1}\left(\bar{z}, x, x_{0}, \alpha\right)^{*}=I_{m}, \\
& \theta_{1}\left(z, x, x_{0}, \alpha\right) \phi_{2}\left(\bar{z}, x, x_{0}, \alpha\right)^{*}-\phi_{1}\left(z, x, x_{0}, \alpha\right) \theta_{2}\left(\bar{z}, x, x_{0}, \alpha\right)^{*}=I_{m} .
\end{aligned}
$$

Finally, we note the connection between $\Phi$ defined in (2.14b) for different boundary value data $\alpha, \gamma \in \mathbb{C}^{m \times 2 m}$ satisfying (2.9), namely

$$
\Phi\left(z, x, x_{0}, \gamma\right)=\Phi\left(z, x, x_{0}, \alpha\right) \alpha \gamma^{*}+\Theta\left(z, x, x_{0}, \alpha\right) \alpha J \gamma^{*} .
$$


This connection formula follows by the uniqueness of solutions of (2.2) and by the identity given in (2.13). It is needed in the proof of Theorem 5.3.

\section{The Leading-Order Term \\ In The Asymptotic Expansion of $M_{ \pm}(z, x, \alpha)$}

Assuming Hypothesis 2.17 the principal result proven in this section will be the following leading-order asymptotic result for half-line Weyl-Titchmarsh matrices $M_{ \pm}\left(z, x_{0}, \alpha_{0}\right)$ associated with the Dirac-type operator (2.76):

$$
M_{ \pm}\left(z, x_{0}, \alpha_{0}\right) \underset{\substack{|z| \rightarrow \infty \\ z \in C_{\varepsilon}}}{=} \pm i I_{m}+o(1) .
$$

Here $\alpha_{0}=\left(I_{m} 0\right) \in \mathbb{C}^{m \times 2 m}$, and $C_{\varepsilon} \subset \mathbb{C}_{+}$denotes the open sector with vertex at zero, symmetry axis along the positive imaginary axis, and opening angle $\varepsilon$, with $0<\varepsilon<\pi / 2$.

This particular topic originates with the order result of Hille [58] and the asymptotic formulas of Everitt [31] and of Everitt and Halvorsen [32]. By appealing to the theory of Riccati equations, Atkinson in [9], 10], and [11] obtained results like those of Hille, Everitt, and Halvorsen, both for the Schrödinger case as well as for the scalar Dirac $(m=1)$ case. Through a deeper understanding of the role played by Riccati theory, Atkinson obtained the first-order asymptotic expansion of $M_{+}\left(z, x, \alpha_{0}\right)$ for the matrix-valued Schrödinger case in an unpublished manuscript [12. Our strategy of proof for 3.1 is patterned after Atkinson's approach, which also appears in our recent work on the full asymptotic expansion for $M_{+}\left(z, x, \alpha_{0}\right)$ in the matrix-valued Schrödinger case [20].

We begin our discussion by noting two additional characterizations for the Weyl disk $\mathcal{D}\left(z, c, x_{0}, \alpha\right)$ for the general Hamiltonian system (2.2a).

Lemma 3.1. Assume Hypotheses 2.1 and 2.2. Let $z \in \mathbb{C} \backslash \mathbb{R}, c \neq x_{0}$, and define $U\left(z, x, x_{0}, \alpha\right)$ in terms of $M \in \mathbb{C}^{m \times m}$ by (2.17). Then $M \in \mathcal{D}\left(z, c, x_{0}, \alpha\right)$ if and only if

$$
\sigma\left(c, x_{0}, z\right) \operatorname{Im}\left(u_{1}\left(z, x, x_{0}, \alpha\right)^{*} u_{2}\left(z, x, x_{0}, \alpha\right)\right)>0, \quad x \in\left[x_{0}, c\right),
$$

or equivalently, if and only if

$$
\sigma\left(c, x_{0}, z\right) \operatorname{Im}\left(u_{2}\left(z, x, x_{0}, \alpha\right) u_{1}\left(z, x, x_{0}, \alpha\right)^{-1}\right)>0, \quad x \in\left[x_{0}, c\right) .
$$

Moreover, $M \in \mathcal{D}_{ \pm}\left(z, x_{0}, \alpha\right)$ if and only if (3.2) and (3.3) hold for $c= \pm \infty$.

Proof. Let $U(z, x)=U\left(z, x, x_{0}, \alpha\right)$, and let $u_{j}(z, x)=u_{j}\left(z, x, x_{0}, \alpha\right), j=1,2$, with $x \in\left[x_{0}, c\right)$. By (2.27),

$$
\begin{aligned}
& 2 \sigma\left(c, x_{0}\right)|\operatorname{Im}(z)| \int_{x}^{c} d s U(z, s)^{*} A(s) U(z, s) \\
& =\left.\sigma\left(x_{0}, c, z\right) U(z, s)^{*}(i J) U(z, s)\right|_{x} ^{c}
\end{aligned}
$$

By 2.18, this yields

$$
\begin{aligned}
& 2 \sigma\left(c, x_{0}, z\right) \operatorname{Im}\left(u_{1}(z, x)^{*} u_{2}(z, x)\right) \\
& =-E_{c}(M)+2 \sigma\left(c, x_{0}\right)|\operatorname{Im}(z)| \int_{x}^{c} d s U(z, s)^{*} A(s) U(z, s) .
\end{aligned}
$$


The integral expression in (3.5) is strictly positive by Hypothesis 2.2. This yields the equivalence of $-E_{c}(M) \geq 0$, and hence of $M \in \mathcal{D}\left(z, c, x_{0}, \alpha\right)$, with the condition given in (3.2). The equivalence of (3.2) and (3.3) follows from the observation that

$$
\operatorname{Im}\left(u_{2}(z, x) u_{1}(z, x)^{-1}\right)=\left(u_{1}(z, x)^{-1}\right)^{*} \operatorname{Im}\left(u_{1}(z, x)^{*} u_{2}(z, x)\right) u_{1}(z, x)^{-1} .
$$

The analogous characterization of $\mathcal{D}_{ \pm}\left(z, x_{0}, \alpha\right)$ now follows from Definition 2.12,

In Lemma 3.1, $u_{j}(z, c), j=1,2$, are well-defined, and $E_{c}(M)=0$ precisely when $\sigma\left(c, x_{0}, z\right) \operatorname{Im}\left(u_{1}(z, c)^{*} u_{2}(z, c)\right)=0$. A similar statement might not hold for (3.3), since $u_{1}\left(z, c, x_{0}, \alpha\right)$ might be singular. In part, the latter point motivates the next characterization of the disk.

Lemma 3.2. Assume Hypotheses [2.1] and [2.2. Let $z \in \mathbb{C} \backslash \mathbb{R}, c \neq x_{0}$, and define $u_{j}(z, x)=u_{j}\left(z, x, x_{0}, \alpha\right), j=1,2$, by (2.17). Then $M \in \mathcal{D}\left(z, c, x_{0}, \alpha\right)$ if and only if

$$
u_{1}(z, x)-i \sigma\left(c, x_{0}, z\right) u_{2}(z, x)
$$

is nonsingular for $x \in\left[x_{0}, c\right]$ and

$$
\begin{aligned}
\vartheta(z, x)=\vartheta\left(z, x, x_{0}, \alpha\right)= & {\left[u_{1}(z, x)+i \sigma\left(c, x_{0}, z\right) u_{2}(z, x)\right] } \\
& \times\left[u_{1}(z, x)-i \sigma\left(c, x_{0}, z\right) u_{2}(z, x)\right]^{-1}
\end{aligned}
$$

satisfies

$$
I_{m}-\vartheta(z, x)^{*} \vartheta(z, x)>0, \quad x \in\left[x_{0}, c\right),
$$

with nonnegativity holding at $x=c$. Moreover, $M \in \mathcal{D}_{ \pm}\left(z, x_{0}, \alpha\right)$ if and only if (3.8) is well-defined on $\left[x_{0}, \pm \infty\right)$ and (3.9) holds for $c= \pm \infty$.

Proof. Let $M \in \mathcal{D}\left(z, c, x_{0}, \alpha\right)$, and suppose that $u_{1}(z, \xi) v=i \sigma\left(c, x_{0}, z\right) u_{2}(z, \xi) v$ for $\xi \in\left[x_{0}, c\right]$ and $v \in \mathbb{C}^{m}, v \neq 0$. Then,

$$
v^{*} \sigma\left(c, x_{0}, z\right) \operatorname{Im}\left(u_{1}(z, \xi)^{*} u_{2}(z, \xi)\right) v=-v^{*} u_{1}(z, \xi)^{*} u_{1}(z, \xi) v .
$$

By (3.2), an immediate contradiction results if $\xi \neq c$. However, if $\xi=c$, then either $v^{*} E_{c}(M) v>0$ or $u_{j}(z, c) v=0, j=1,2$. In either case, a contradiction results, since $E_{c}(M) \leq 0$ by Definition 2.7 and $U=\left(u_{1}^{t}, u_{2}^{t}\right)^{t}$ satisfies the firstorder system (2.2a). Hence, $\vartheta(z, x)$ is well-defined on $\left[x_{0}, c\right]$. For $x \in\left[x_{0}, c\right)$ and $M \in \mathcal{D}\left(z, c, x_{0}, \alpha\right)$, (3.2) implies that

$$
2 i \sigma\left(c, x_{0}, z\right)\left(u_{1}(z, x)^{*} u_{2}(z, x)-u_{2}(z, x)^{*} u_{1}(z, x)\right)<0 .
$$

This is equivalent to

$$
\begin{aligned}
& {\left[u_{1}(z, x)^{*}-i \sigma\left(c, x_{0}, z\right) u_{2}(z, x)^{*}\right]\left[u_{1}(z, x)+i \sigma\left(c, x_{0}, z\right) u_{2}(z, x)\right]} \\
& \quad<\left[u_{1}(z, x)^{*}+i \sigma\left(c, x_{0}, z\right) u_{2}(z, x)^{*}\right]\left[u_{1}(z, x)-i \sigma\left(c, x_{0}, z\right) u_{2}(z, x)\right]
\end{aligned}
$$

on $\left[x_{0}, c\right)$. Given the nonsingularity of $u_{1}(z, x)-i \sigma\left(c, x_{0}, z\right) u_{2}(z, x)$ on $\left[x_{0}, c\right]$, (3.12) implies (3.9), with nonnegativity holding at $x=c$.

Next, let $M \in \mathbb{C}^{m \times m}$, and suppose that $\vartheta(z, x)$, defined by (3.8), is well-defined on $\left[x_{0}, c\right]$, and satisfies (3.9). Then, on $\left[x_{0}, c\right),(3.12)$ and consequently (3.11) follow, which implies that (3.2) holds, and hence that $M \in \mathcal{D}\left(z, c, x_{0}, \alpha\right)$. The analogous characterization of $\mathcal{D}_{ \pm}\left(z, x_{0}, \alpha\right)$ follows from Definition [2.12,

By Lemma 3.1 one notes, for $z \in \mathbb{C} \backslash \mathbb{R}$, that $M \in \mathcal{D}\left(z, c, x_{0}, \alpha\right)$ if and only if

$$
V\left(z, x, x_{0}, \alpha\right)=u_{2}\left(z, x, x_{0}, \alpha\right) u_{1}\left(z, x, x_{0}, \alpha\right)^{-1}, \quad x \in\left[x_{0}, c\right),
$$


is well-defined while satisfying

$$
\sigma\left(c, x_{0}, z\right) \operatorname{Im}\left(V\left(z, x, x_{0}, \alpha\right)\right)>0, \quad x \in\left[x_{0}, c\right) .
$$

In terms of $V\left(z, x, x_{0}, \alpha\right)$ and by (3.8), one notes that

$$
\begin{aligned}
\vartheta\left(z, x, x_{0}, \alpha\right)= & {\left[I_{m}+i \sigma\left(c, x_{0}, z\right) V\left(z, x, x_{0}, \alpha\right)\right] } \\
& \times\left[I_{m}-i \sigma\left(c, x_{0}, z\right) V\left(z, x, x_{0}, \alpha\right)\right]^{-1}, \quad x \in\left[x_{0}, c\right),
\end{aligned}
$$

is a Cayley-type transformation of $V\left(z, x, x_{0}, \alpha\right)$. In the scalar context, this transformation corresponds to a conformal mapping of the complex upper half-plane to the unit disk. Moreover, defined as it is, $V\left(z, x, x_{0}, \alpha\right)$ satisfies a Riccati differential equation that is associated with the Hamiltonian system (2.2a), while $\vartheta\left(z, x, x_{0}, \alpha\right)$ satisfies a Riccati equation obtained by the Cayley-type transformation (3.15) applied to the differential equation satisfied by $V\left(z, x, x_{0}, \alpha\right)$.

For the Dirac-type case of (2.2a $)$, one observes by a simple calculation that $V\left(z, x, x_{0}, \alpha_{0}\right)$ satisfies a particular initial value problem for a Riccati differential equation.

Lemma 3.3. Assume Hypotheses 2.1, 2.2, and 2.17. Let $\alpha_{0}=\left(I_{m} 0\right) \in \mathbb{C}^{m \times 2 m}$, let $u_{j}(z, x)=u_{j}\left(z, x, x_{0}, \alpha_{0}\right), j=1,2$, be defined by (2.17), and suppose that $V\left(z, x, x_{0}, \alpha_{0}\right)$ is well-defined by (3.13). Then, $V(z, \cdot)=V\left(z, \cdot, x_{0}, \alpha_{0}\right)$ satisfies

$$
\begin{gathered}
V^{\prime}(z, x)+z V(z, x)^{2}+V(z, x) B_{2,2}(x) V(z, x) \\
+B_{1,2}(x) V(z, x)+V(z, x) B_{2,1}(x)+B_{1,1}(x)+z I_{m}=0 \\
V\left(z, x_{0}\right)=M,
\end{gathered}
$$

where $B_{j, k} \in \mathbb{C}^{m \times m}, j, k=1,2$, are defined in (2.1d).

Hence, by Lemma 3.1, the associated relations (3.13) and (3.14), and the uniqueness of solutions for (3.16), we obtain the following result for the Dirac-type case.

Theorem 3.4. Assume Hypotheses [2.1, 2.2, and 2.17, and let $\alpha_{0}=\left(I_{m} 0\right) \in$ $\mathbb{C}^{m \times 2 m}$. Then, $M \in \mathcal{D}\left(z, c, x_{0}, \alpha_{0}\right)$ if and only if the initial value problem given by (3.16) has a solution $V(z, \cdot)$, well-defined and satisfying

$$
\sigma\left(c, x_{0}, z\right) \operatorname{Im}(V(z, x))>0, \quad x \in\left[x_{0}, c\right) .
$$

Moreover, $M \in \mathcal{D}_{ \pm}\left(z, x_{0}, \alpha_{0}\right)$ if and only if 3.17) holds for $c= \pm \infty$.

Remark 3.5. An important consequence of Theorem 3.4 and the uniqueness of solutions for (3.16) is that solution trajectories for (3.16) that satisfy (3.17) consist of elements of Weyl disks; that is,

$$
V\left(z, x, x_{0}, \alpha_{0}\right) \in \mathcal{D}\left(z, c, x, \alpha_{0}\right), \quad x \in\left[x_{0}, c\right) .
$$

Given the characterization of $\mathcal{D}\left(z, c, x_{0}, \alpha_{0}\right)$ in Definition 2.7A, for each $x \in\left[x_{0}, c\right)$ there is a $\beta \in \mathbb{C}^{m \times 2 m}$ with $\sigma\left(c, x_{0}, z\right) \operatorname{Im}\left(\beta_{2} \beta_{1}^{*}\right) \geq 0$, such that

$$
V\left(z, x, x_{0}, \alpha_{0}\right)=M\left(z, c, x, \alpha_{0}, \beta\right) .
$$

It is in this sense that we let $M\left(z, c, x, \alpha_{0}\right)$ denote our solution of the initial value problem (3.16) that satisfies (3.17). Analogously,

$$
V\left(z, x, x_{0}, \alpha_{0}\right) \in \mathcal{D}_{ \pm}\left(z, x, \alpha_{0}\right), \quad x \in\left[x_{0}, \pm \infty\right),
$$

for trajectories of (3.16) that satisfy (3.17) for $c= \pm \infty$. Hence, in this sense, we let $M_{ \pm}\left(z, x, \alpha_{0}\right)$ denote those solutions of (3.16) that satisfy (3.17) for $c= \pm \infty$. 
However, by Lemma 2.15 our Dirac system is in the limit point case at $\pm \infty$. Each $\mathcal{D}_{ \pm}\left(z, x, \alpha_{0}\right)$ consists of a unique matrix, and thus $M_{ \pm}\left(z, x, \alpha_{0}\right)$ describes unique trajectories for (3.16a). This contrasts with the matrix-valued Schrödinger case considered in [20], where there are as many trajectories, each denoted by either $M_{+}\left(z, x, \alpha_{0}\right)$ or $M_{-}\left(z, x, \alpha_{0}\right)$, as there are matrices in a given initial disk $\mathcal{D}_{ \pm}\left(z, x_{0}, \alpha_{0}\right)$.

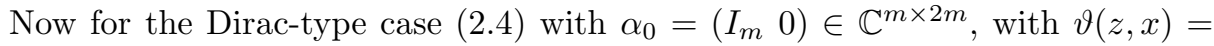
$\vartheta\left(z, x, x_{0}, \alpha_{0}\right)$ defined in (3.8) and (3.15), and with $x \in\left[x_{0}, c\right)$, one concludes that

$$
\vartheta(z, x)\left[u_{1}(z, x)-i \sigma\left(c, x_{0}, z\right) u_{2}(z, x)\right]=u_{1}(z, x)+i \sigma\left(c, x_{0}, z\right) u_{2}(z, x),
$$

and hence that

$$
\begin{aligned}
& I_{m}+\vartheta(z, x)=2 u_{1}(z, x)\left[u_{1}(z, x)-i \sigma\left(c, x_{0}, z\right) u_{2}(z, x)\right]^{-1} \\
& I_{m}-\vartheta(z, x)=-2 i \sigma\left(c, x_{0}, z\right) u_{2}(z, x)\left[u_{1}(z, x)-i \sigma\left(c, x_{0}, z\right) u_{2}(z, x)\right]^{-1} .
\end{aligned}
$$

Differentiating (3.21), one obtains

$$
\begin{aligned}
\vartheta^{\prime}\left(u_{1}-i \sigma u_{2}\right)= & \left(I_{m}-\vartheta\right)\left(z u_{2}+B_{2,1} u_{1}+B_{2,2} u_{2}\right) \\
& +i \sigma\left(I_{m}+\vartheta\right)\left(-z u_{1}-B_{1,1} u_{1}-B_{1,2} u_{2}\right) .
\end{aligned}
$$

By (3.22) one concludes that $\vartheta\left(z, \cdot, x_{0}, \alpha_{0}\right)$ satisfies the initial value problem given by

$$
\vartheta^{\prime}(z, x)=\frac{1}{2}\left(\begin{array}{c}
I_{m}+\vartheta(z, x)^{t} \\
I_{m}-\vartheta(z, x)^{t}
\end{array}\right)^{t}
$$

$$
\begin{aligned}
\times & \left(\begin{array}{cc}
-i \sigma\left(c, x_{0}, z\right)\left(z I_{m}+B_{1,1}(x)\right) & B_{1,2}(x) \\
B_{2,1}(x) & i \sigma\left(c, x_{0}, z\right)\left(z I_{m}+B_{2,2}(x)\right)
\end{array}\right) \\
\times & \left(\begin{array}{c}
I_{m}+\vartheta(z, x) \\
I_{m}-\vartheta(z, x)
\end{array}\right), \\
& \vartheta\left(z, x_{0}\right)=\left(I_{m}+i \sigma\left(c, x_{0}, z\right) M\right)\left(I_{m}-i \sigma\left(c, x_{0}, z\right) M\right)^{-1},
\end{aligned}
$$

where $B_{j, k} \in \mathbb{C}^{m \times m}, j, k=1,2$, satisfy Hypothesis 2.1

By Lemma 3.2 and the uniqueness of solutions for (3.24), one obtains the following result in the Dirac-type case (2.4).

Theorem 3.6. Assume Hypothesis 2.17. Then $M \in \mathcal{D}\left(z, c, x_{0}, \alpha_{0}\right)$ if and only if the initial value problem given by (3.24) has a solution $\vartheta(z, \cdot)$ that is well-defined on $\left[x_{0}, c\right]$ and satisfies

$$
I_{m}-\vartheta(z, x)^{*} \vartheta(z, x)>0, \quad x \in\left[x_{0}, c\right) .
$$

Moreover, $M \in \mathcal{D}_{ \pm}\left(z, x_{0}, \alpha_{0}\right)$ if and only if (3.25) holds for $c= \pm \infty$.

Given the positivity present in (3.25), we note the exact correspondence which exists, by (3.15), between solutions of (3.16) that satisfy (3.17) and those solutions of (3.24) that satisfy (3.25). In particular, given Remark 3.5 we rewrite (3.15) as

$$
\begin{aligned}
\vartheta\left(z, x, x_{0}, \alpha_{0}\right)= & {\left[I_{m}+i \sigma\left(c, x_{0}, z\right) M\left(z, c, x, \alpha_{0}\right)\right] } \\
& \times\left[I_{m}-i \sigma\left(c, x_{0}, z\right) M\left(z, c, x, \alpha_{0}\right)\right]^{-1}, \quad x \in\left[x_{0}, c\right),
\end{aligned}
$$

Moreover, our Dirac system is in the limit point case at $\pm \infty$. Consequently, there are unique solutions of (3.24), $\vartheta_{ \pm}\left(z, \cdot, x_{0}, \alpha_{0}\right), z \in \mathbb{C} \backslash \mathbb{R}$, that satisfy (3.25) for 
$c= \pm \infty$, and that correspond to the unique solutions of (3.16), $M_{ \pm}\left(z, x, \alpha_{0}\right)$, and that satisfy (3.17) for $c= \pm \infty$; specifically,

$$
\vartheta_{ \pm}\left(z, x, x_{0}, \alpha_{0}\right)=\left[I_{m} \pm i \sigma(z) M_{ \pm}\left(z, x, \alpha_{0}\right)\right]\left[I_{m} \mp i \sigma(z) M_{ \pm}\left(z, x, \alpha_{0}\right)\right]^{-1} .
$$

These relationships form the basis for the analysis to follow. The asymptotic result (3.1) is obtained by an analysis of the corresponding asymptotic behavior for all solutions $\vartheta\left(z, \cdot, x_{0}, \alpha_{0}\right)$ described in (3.24); these include among them the particular solutions $\vartheta_{ \pm}\left(z, \cdot, x_{0}, \alpha_{0}\right)$. Thus asymptotic behavior is deduced for all corresponding solutions $M\left(z, c, \cdot, \alpha_{0}\right)$ of (3.16), which include among them the solutions $M_{ \pm}\left(z, \cdot, \alpha_{0}\right)$. The advantage of this approach comes from the compactification inherent in the Cayley-type transformation (3.26), and the resulting boundedness of the solutions as a consequence of (3.25).

We pause for a moment to address, in the following remark, a point raised by us in [20] for the matrix-valued Schrödinger case described in (2.5).

Remark 3.7. With $u_{j}(z, x)=u_{j}\left(z, x, x_{0}, \alpha\right), j=1,2$, defined in (2.17) for the general Hamiltonian system (2.2a), an analog to Lemma 3.2 for the characterization of $\mathcal{D}\left(z, c, x_{0}, \alpha\right)$ is obtained by replacing the expression in (3.7) with

$$
u_{1}(z, x)-i|z|^{-1 / 2} \sigma\left(c, x_{0}, z\right) u_{2}(z, x)
$$

and by replacing the definition for $\vartheta(z, x)=\vartheta\left(z, x, x_{0}, \alpha\right)$ given in (3.8) with

$$
\begin{aligned}
\vartheta(z, x)= & \left(u_{1}(z, x)+i|z|^{-1 / 2} \sigma\left(c, x_{0}, z\right) u_{2}(z, x)\right) \\
& \times\left(u_{1}(z, x)-i|z|^{-1 / 2} \sigma\left(c, x_{0}, z\right) u_{2}(z, x)\right)^{-1} .
\end{aligned}
$$

Specific to the matrix-valued Schrödinger case, we obtain analogs of Lemma 3.3 Theorem 3.4, and Theorem 3.6 by replacing equation 3.16a with

$$
V^{\prime}(z, x)+V(z, x)^{2}-Q(x)+z I_{m}=0
$$

and by replacing the equations in (3.24) with

$$
\begin{aligned}
& \vartheta^{\prime}(z, x)= \sigma\left(c, x_{0}, z\right) \frac{1}{2}\left(\begin{array}{c}
I_{m}+\vartheta(z, x)^{t} \\
I_{m}-\vartheta(z, x)^{t}
\end{array}\right)^{t}\left(\begin{array}{cc}
-i|z|^{-1 / 2}\left(z I_{m}-Q(x)\right) & 0 \\
0 & i|z|^{-1 / 2} I_{m}
\end{array}\right) \\
&(3.31 \mathrm{a}) \quad \times\left(\begin{array}{c}
I_{m}+\vartheta(z, x) \\
I_{m}-\vartheta(z, x)
\end{array}\right), \\
&(3.31 \mathrm{~b}) \quad \vartheta\left(z, x_{0}\right)=\left(I_{m}+i|z|^{-1 / 2} \sigma\left(c, x_{0}, z\right) M\right)\left(I_{m}-i|z|^{-1 / 2} \sigma\left(c, x_{0}, z\right) M\right)^{-1} .
\end{aligned}
$$

$\mathcal{D}^{\mathcal{R}}\left(z, c, x_{0}, \alpha_{0}\right)$ was defined in $\left[20\right.$ to be the set of those $M \in \mathbb{C}^{m \times m}$ for which the intial value problem given by (3.31) has a solution, $\vartheta(z, x)$, which is well-defined on $\left[x_{0}, c\right]$ and satisfies 3.25 . In 20 ] we showed that $\mathcal{D}\left(z, c, x_{0}, \alpha_{0}\right) \subseteq \mathcal{D}^{\mathcal{R}}\left(z, c, x_{0}, \alpha_{0}\right)$. This was sufficient for the subsequent analysis in [20. However, as the analog of Theorem 3.6 now shows, one actually has equality of the two disks in 20], that is,

$$
\mathcal{D}\left(z, c, x_{0}, \alpha_{0}\right)=\mathcal{D}^{\mathcal{R}}\left(z, c, x_{0}, \alpha_{0}\right)
$$

To obtain a proof of (3.1) for the Dirac-type case, we adapt an approach due to Atkinson [12] for proving a result analogous to (3.1) for the matrix-valued Schrödinger case (cf., e.g., [20, Theorem 3.1]). In light of Remark [3.12, we begin by restricting our attention to $z \in \mathbb{C}_{+}$, and as in the previous discussion, take $\alpha_{0}=\left(I_{m} 0\right) \in \mathbb{C}^{m \times 2 m}$. 
First we introduce two systems related to (3.24) by means of a change of variables. Let

$$
\varphi(z, t)=\vartheta(z, x), \quad t=\left(x-x_{0}\right)|z|, \quad x \in\left[x_{0}, c\right) .
$$

With this change, (3.24) becomes

$$
\begin{aligned}
\varphi^{\prime}(z, t)= & \frac{1}{2}|z|^{-1}\left(\begin{array}{c}
I_{m}+\varphi(z, t)^{t} \\
I_{m}-\varphi(z, t)^{t}
\end{array}\right)^{t}\left(\begin{array}{cc}
\mp i\left(z I_{m}+\widetilde{B}_{1,1}(t)\right) & \widetilde{B}_{1,2}(t) \\
\widetilde{B}_{2,1}(t) & \pm i\left(z I_{m}+\widetilde{B}_{2,2}(t)\right)
\end{array}\right) \\
& \times\left(\begin{array}{l}
I_{m}+\varphi(z, t) \\
I_{m}-\varphi(z, t)
\end{array}\right) .
\end{aligned}
$$

With $M=M\left(z, c, x_{0}, \alpha_{0}\right) \in \mathcal{D}\left(z, c, x_{0}, \alpha_{0}\right)$, (3.24b) becomes

$$
\varphi(z, 0)=\left(i I_{m} \mp M\left(z, c, x_{0}, \alpha_{0}\right)\right)\left(i I_{m} \pm M\left(z, c, x_{0}, \alpha_{0}\right)\right)^{-1},
$$

and (3.25) becomes

$$
\varphi(z, t)^{*} \varphi(z, t)<I_{m}, \quad t \in\left[0,\left(c-x_{0}\right)|z|\right),
$$

where in 3.34a),

$$
\widetilde{B}_{j, k}(t)=B_{j, k}\left(x_{0}+t|z|^{-1}\right), \quad j, k=1,2 .
$$

In the complete system (3.34), one now has a set of conditions equivalent to system (3.24) and (3.25).

We recall that $C_{\varepsilon} \subset \mathbb{C}_{+}$represents the open sector with vertex at zero, symmetry axis along the positive imaginary axis, and opening angle $\varepsilon$, with $0<\varepsilon<\pi / 2$. Next, consider a sequence $z_{n} \in \mathbb{C}_{\varepsilon}, n \in \mathbb{N}$, such that $\left|z_{n}\right| \rightarrow \infty$ as $n \rightarrow \infty$ and such that

$$
0<\varepsilon<\delta_{n}=\arg \left(z_{n}\right)<\pi-\varepsilon .
$$

By choosing an appropriate subsequence, we may assume that

$$
\delta_{n} \rightarrow \delta \in[\varepsilon, \pi-\varepsilon] .
$$

Let $\varphi\left(z_{n}, t\right)$ denote a corresponding sequence of functions that satisfy (3.34a and (3.34c), with initial data $\varphi\left(z_{n}, 0\right)$ defined by 3.34b for a sequence of points $M\left(z_{n}, c, x_{0}, \alpha_{0}\right)$, where each $M\left(z_{n}, c, x_{0}, \alpha_{0}\right)$ is chosen to be an element of the disk $\mathcal{D}\left(z_{n}, c, x_{0}, \alpha_{0}\right)$. Note that as $z_{n} \rightarrow \infty$, the intervals described in (3.34c) eventually cover all compact subintervals of $\mathbb{R}_{+}$. Given the uniform boundedness of $\varphi_{n}(t)=\varphi\left(z_{n}, t\right)$ described in (3.34c), we assume, upon passing to an appropriate subsequence still denoted by $\varphi_{n}(0)$, that

$$
\varphi_{n}(0)=\varphi\left(z_{n}, 0\right) \rightarrow \varphi_{ \pm}(\delta), \text { for } \pm\left(c-x_{0}\right)>0 \text { as } n \rightarrow \infty,
$$

and as a consequence, that

$$
\varphi_{ \pm}(\delta)^{*} \varphi_{ \pm}(\delta) \leq I_{m}
$$

With $\varphi_{ \pm}(\delta)$ defined in (3.37) as $\left|z_{n}\right| \rightarrow \infty$, we consider limiting systems associated with (3.34):

$$
\eta_{ \pm}^{\prime}(t)=\frac{1}{2}\left(\begin{array}{l}
I_{m}+\eta_{ \pm}(t)^{t} \\
I_{m}-\eta_{ \pm}(t)^{t}
\end{array}\right)^{t}\left(\begin{array}{cc}
\mp i e^{i \delta} I_{m} & 0 \\
0 & \pm i e^{i \delta} I_{m}
\end{array}\right)\left(\begin{array}{l}
I_{m}+\eta_{ \pm}(t) \\
I_{m}-\eta_{ \pm}(t)
\end{array}\right), \quad \pm t \geq 0,
$$

$$
\eta_{ \pm}(0)=\varphi_{ \pm}(\delta)
$$


Theorem 3.8. Assume Hypothesis 2.17. Then the solution $\eta_{ \pm}$of (3.39) satisfies

$$
\eta_{ \pm}(t)^{*} \eta_{ \pm}(t) \leq I_{m}, \quad t \in[0, \pm \infty)
$$

Moreover, the solutions $\varphi_{n}=\varphi\left(z_{n}, \cdot\right)$ of (3.34) converge to $\eta_{ \pm}$uniformly on $[0, \pm T]$ for every $T>0$, as $n \rightarrow \infty$.

Proof. In this proof, we consider only the case corresponding to $t \geq 0$, that is, $\eta_{+}(0)=\varphi_{+}(\delta)$ in (3.39b). The other case follows in a similar manner. For this reason, we let $\eta(t)=\eta_{+}(t)$ in the remaining discussion. We also let $T \in \mathbb{R}_{+}$be the greatest value such that (3.40) holds for $t \in[0, T]$, and show that (3.40) must hold for some $\left[0, T^{\prime}\right]$ with $T^{\prime}>T$, thus proving $T=\infty$.

The solution of (3.39), $\eta$, presumed to be defined on $[0, T]$, can be continued onto some $\left[0, T^{\prime}\right]$ with $T^{\prime}>T ; \eta$ then satisfies

$$
\eta(t)^{*} \eta(t) \leq \kappa^{2} I_{m}
$$

for $0 \leq t \leq T^{\prime}$ and for some $\kappa \geq 1$

For brevity, let $\varphi_{n}^{\prime}(t)=G_{n}\left(\varphi_{n}, t\right)$ denote (3.34a) with $z=z_{n}$, and let $\eta^{\prime}(t)=$ $H(\eta, t)$ denote (3.39a) in the following. Integrating (3.39a) and (3.34a), one obtains

$$
\begin{aligned}
\varphi_{n}(t)-\eta(t)= & \varphi_{n}(0)-\varphi_{0}(\delta)+\int_{0}^{t} d s\left\{G_{n}(\eta, s)-H(\eta, s)\right\} \\
& +\int_{0}^{t} d s\left\{G_{n}\left(\varphi_{n}, s\right)-G_{n}(\eta, s)\right\} .
\end{aligned}
$$

We note that

$$
\begin{aligned}
G_{n}(\eta, s)-H(\eta, s)= & \frac{1}{2} i\left(e^{i \delta}-e^{i \delta_{n}}\right)\left(I_{m}+\eta(s)\right)^{2}-\frac{1}{2} i\left(e^{i \delta}-e^{i \delta_{n}}\right)\left(I_{m}-\eta(s)\right)^{2} \\
& +\sum_{j, k=1}^{2} F_{j, k}\left(z_{n}, s\right)
\end{aligned}
$$

where

$$
\begin{aligned}
& F_{1,1}\left(z_{n}, s\right)=-\frac{1}{2} i\left|z_{n}\right|^{-1}\left(I_{m}+\eta(s)\right) \widetilde{B}_{1,1}(s)\left(I_{m}+\eta(s)\right), \\
& F_{2,2}\left(z_{n}, s\right)=\frac{1}{2} i\left|z_{n}\right|^{-1}\left(I_{m}-\eta(s)\right) \widetilde{B}_{2,2}(s)\left(I_{m}-\eta(s)\right), \\
& F_{1,2}\left(z_{n}, s\right)=\frac{1}{2} i\left|z_{n}\right|^{-1}\left(I_{m}+\eta(s)\right) \widetilde{B}_{1,2}(s)\left(I_{m}-\eta(s)\right), \\
& F_{2,1}\left(z_{n}, s\right)=\frac{1}{2} i\left|z_{n}\right|^{-1}\left(I_{m}-\eta(s)\right) \widetilde{B}_{2,1}(s)\left(I_{m}+\eta(s)\right) .
\end{aligned}
$$

Thus, for $t \in\left[0, T^{\prime}\right]$, (3.41) implies that as $n \rightarrow \infty$

$$
\left|e^{i \delta}-e^{i \delta_{n}}\right| \int_{0}^{t}\left\|I_{m} \pm \eta(s)\right\|_{\mathbb{C}^{m \times m}}^{2} d s=o(1),
$$

and together with (3.33) and (3.34d) that

$$
\int_{0}^{t}\left\|F_{j, k}(s)\right\|_{\mathbb{C}^{m \times m}} d s=O\left(\int_{x_{0}}^{x_{0}+t\left|z_{n}\right|^{-1}}\left\|\widetilde{B}_{j, k}(s)\right\|_{\mathbb{C}^{m \times m}} d s\right)=o(1) .
$$


(Here $\|\cdot\|_{\mathbb{C}^{m \times m}}$ denotes a norm on $\mathbb{C}^{m \times m}$.) Hence, by (3.43) -(3.46), one infers that for $t \in\left[0, T^{\prime}\right]$ and as $n \rightarrow \infty$,

$$
\int_{0}^{t}\left\{G_{n}(\eta, s)-H(\eta, s)\right\} d s=o(1) .
$$

Next, one notes that

$$
G_{n}\left(\varphi_{n}, s\right)-G_{n}(\eta, s)=2 i e^{i \delta_{n}}\left(\eta(s)-\varphi_{n}(s)\right)+\sum_{j, k=1}^{2} K_{j, k}\left(z_{n}, s\right),
$$

where

$$
K_{1,1}\left(z_{n}, s\right)=\frac{-i}{2}\left|z_{n}\right|^{-1}\left\{\left(I_{m}+\varphi_{n}\right) B_{1,1}(s)\left(\varphi_{n}-\eta\right)+\left(\varphi_{n}-\eta\right) B_{1,1}(s)\left(I_{m}+\eta\right)\right\},
$$

$$
K_{2,2}\left(z_{n}, s\right)=\frac{i}{2}\left|z_{n}\right|^{-1}\left\{\left(I_{m}-\varphi_{n}\right) B_{2,2}(s)\left(\eta-\varphi_{n}\right)+\left(\eta-\varphi_{n}\right) B_{2,2}(s)\left(I_{m}-\eta\right)\right\},
$$

$$
K_{1,2}\left(z_{n}, s\right)=\frac{1}{2}\left|z_{n}\right|^{-1}\left\{\left(I_{m}+\varphi_{n}\right) B_{1,2}(s)\left(\eta-\varphi_{n}\right)+\left(\varphi_{n}-\eta\right) B_{1,2}(s)\left(I_{m}-\eta\right)\right\},
$$

$$
K_{2,1}\left(z_{n}, s\right)=\frac{1}{2}\left|z_{n}\right|^{-1}\left\{\left(I_{m}-\varphi_{n}\right) B_{2,1}(s)\left(\varphi_{n}-\eta\right)+\left(\eta-\varphi_{n}\right) B_{2,1}(s)\left(I_{m}+\eta\right)\right\} .
$$

By (3.38) and (3.41), for $s \in\left[0, T^{\prime}\right]$,

$$
\left\|I_{m} \pm \varphi_{n}(s)\right\|_{\mathbb{C}^{m \times m}} \leq 2, \quad\left\|I_{m} \pm \eta(s)\right\|_{\mathbb{C}^{m \times m}} \leq \kappa+1,
$$

and hence by (3.48)-(3.50),

$$
\begin{aligned}
& \left\|G_{n}\left(\varphi_{n}, s\right)-G_{n}(\eta, s)\right\|_{\mathbb{C}^{m \times m}} \\
& \leq\left\|\eta(s)-\varphi_{n}(s)\right\|_{\mathbb{C}^{m \times m}}\left\{2+\frac{\left|z_{n}\right|^{-1}}{2}(3+\kappa) \sum_{j, k=1}^{2}\left\|\widetilde{B}_{j, k}(s)\right\|_{\mathbb{C}^{m \times m}}\right\} .
\end{aligned}
$$

Of course, by (3.37) as $n \rightarrow \infty$,

$$
\phi_{n}(0)-\phi_{+}(\delta)=o(1) .
$$

Thus, by (3.46), (3.51) and (3.52), one concludes for $t \in\left[0, T^{\prime}\right]$ and as $n \rightarrow \infty$, that

$$
\begin{aligned}
& \left\|\varphi_{n}(t)-\eta(t)\right\|_{\mathbb{C}^{m \times m}} \leq o(1) \\
& +\int_{0}^{t}\left\|\varphi_{n}(s)-\eta(s)\right\|_{\mathbb{C}^{m \times m}}\left\{2+\frac{\left|z_{n}\right|^{-1}}{2}(3+\kappa) \sum_{j, k=1}^{2}\left\|\widetilde{B}_{j, k}(s)\right\|_{\mathbb{C}^{m \times m}}\right\} d s .
\end{aligned}
$$

Gronwall's inequality applied to (3.53) together with a consideration of the effect of the variable change (3.33), as illustrated in (3.46), yields

$$
\varphi_{n}(t)-\eta(t) \rightarrow 0 \text { as } n \rightarrow \infty
$$

uniformly for $t \in\left[0, T^{\prime}\right]$. Thus by (3.34c), we get the contradiction that for all $t \in\left[0, T^{\prime}\right], \eta$ satisfies (3.40).

What solutions of (3.39) satisfy (3.40)? 
Lemma 3.9. Assume Hypothesis 2.17. If $\eta_{ \pm}$is a solution of (3.39a) that satisfies (3.40), then

$$
0=\eta_{ \pm}(t), \quad t \in[0, \pm \infty) .
$$

Proof. We note that 3.39a is equivalent to 3.34a with $\widetilde{B}=0$. By the variable change (3.33), (3.39a) is also equivalent to (3.24a) with $B=0$. Next, we recall the connection between the Riccati-type equations (3.24a), and (3.16a) by means of the Cayley transformation (3.26). Solution matrices of (3.39a) that statisfy (3.40) at $t=0$ thus correspond to solution matrices $V(z, \cdot)$ of (3.16a) for which $\operatorname{Im}\left(V\left(z, x_{0}\right)\right) \geq 0$. Moreover, solutions of (3.16a) for which $\operatorname{Im}\left(V\left(z, x_{0}\right)\right) \geq 0$ are obtainable from solutions of (2.2a) with $B=0$, by means of (2.17) with $\operatorname{Im}(M) \geq 0$. Thus, by utilizing this connection between explicit exponential solutions of (2.2a) with $B=0$ and solutions of the Riccati-type equation (3.16a), and by performing on the resulting solution of (3.16a) the conformal mapping (3.26) followed by the variable transformation (3.33), one obtains the following solution for (3.34a):

$$
\varphi(z, t)=\left(i I_{m} \mp M\right)\left(i I_{m} \pm M\right)^{-1} \exp \left(\mp 2 i t e^{i \delta}\right),
$$

for $\pm t \geq 0, \operatorname{Im}( \pm M) \geq 0$, and $z \in \mathbb{C}_{+}$. By hypothesis, $0<\delta<\pi$. Thus the exponential term in (3.56) will result in

$$
\|\varphi(z, t)\|_{\mathbb{C}^{m \times m}}>1 \text { as } t \rightarrow \pm \infty
$$

unless

$$
M= \pm i I_{m}
$$

thus implying (3.55).

One then obtains the following result.

Corollary 3.10. With $\phi_{ \pm}(\delta)$ defined in (3.37), $\eta_{ \pm}(0)=\phi_{ \pm}(\delta)=0$.

For $M\left(z_{n}, c, x_{0}, \alpha_{0}\right) \in \mathcal{D}\left(z_{n}, c, x_{0}, \alpha_{0}\right)$, it follows by (3.34b), (3.37), and Corollary 3.10 that

$$
\left[i I_{m} \mp M\left(z_{n}, c, x_{0}, \alpha_{0}\right)\right]\left[i I_{m} \pm M\left(z_{n}, c, x_{0}, \alpha_{0}\right)\right]^{-1}=o(1), \quad \pm\left(c-x_{0}\right)>0,
$$

as $n \rightarrow \infty$. Hence one infers, for elements of $\mathcal{D}\left(z_{n}, c, x_{0}, \alpha_{0}\right)$, that

$$
M\left(z_{n}, c, x_{0}, \alpha_{0}\right)= \pm i I_{m}+o(1), \quad \pm\left(c-x_{0}\right)>0,
$$

as $|z| \rightarrow \infty$ in $C_{\varepsilon}$. This proves (3.1). Actually, (3.60) is a statement for all elements of $\mathcal{D}\left(z, c, x_{0}, \alpha_{0}\right)$ including the particular element $M_{ \pm}\left(z, x_{0}, \alpha_{0}\right)$, for $\pm\left(c-x_{0}\right)>0$.

In (3.1) an asymptotic expansion is given that is uniform with respect to $\arg (z)$ for $|z| \rightarrow \infty$ in $C_{\varepsilon}$. We now vary the reference point, $x_{0}$, and observe that the asymptotic expansion in (3.1) is also uniform with respect to $x_{0}$ whenever $x_{0}$ is confined to a compact subset of $\mathbb{R}$.

Theorem 3.11. Assume Hypothesis 2.17. Let $\alpha_{0}=\left(I_{m} 0\right) \in \mathbb{C}^{m \times 2 m}$, and denote by $C_{\varepsilon} \subset \mathbb{C}_{+}$the open sector with vertex at zero, symmetry axis along the positive imaginary axis and opening angle $\varepsilon$, with $0<\varepsilon<\pi / 2$. Let $M_{ \pm}\left(z, x_{0}, \alpha_{0}\right)$ be the unique elements of the limit disks $\mathcal{D}_{ \pm}\left(z, x_{0}, \alpha_{0}\right)$ for the Dirac system given by (2.2) and (2.4). Then,

$$
M_{ \pm}\left(z, x, \alpha_{0}\right) \underset{\substack{|z| \rightarrow \infty \\ z \in C_{\varepsilon}}}{=} \pm i I_{m}+o(1)
$$


uniformly with respect to $\arg (z)$, for $|z| \rightarrow \infty$ in $C_{\varepsilon}$, and uniformly with respect to $x$, as long as $x$ varies in compact subsets of $\left[x_{0}, \pm \infty\right)$.

Proof. We note that the system (3.39) is independent of the reference point $x_{0}$. Next, we recall that $\delta$, defined in (3.36) is determined by an a priori choice of the sequence $z_{n}$, subject only to $z_{n}$ being in $C_{\varepsilon}$ (cf. (3.35)). Moreover, we note that $\varphi_{ \pm}(\delta)$, defined as a limit in (3.37), described explicity in Corollary 3.10, and which gives solutions of (3.39) satisfying (3.40) for $t \in[0, \pm \infty)$, is also independent of the reference point $x_{0}$. Thus, had we chosen a different point of reference, $x_{0}^{\prime} \neq x_{0}$, at the start, the asymptotic analysis begun in Theorem 3.8 and continued through (3.59) would remain the same after the variable change in (3.33), except for the integral expression in (3.46), in which $x_{0}$ would be replaced by $x_{0}^{\prime}$. However, given the local integrability assumption on $B$ in Hypothesis 2.1, one concludes that this integral expression is uniformly continuous with respect to $x_{0}$ whenever $x_{0}$ is confined to a compact subset of $\mathbb{R}$. Thus (3.46), and consequently (3.54), are uniform with respect to $t$ and with respect to $x_{0}$ whenever both are confined to compact subsets of $\mathbb{R}$. Consequently, (3.59) holds for elements $\mathcal{D}\left(z, c, x_{0}, \alpha_{0}\right)$, this asymptotic expansion is uniform with respect to $\arg (z)$ for $|z| \rightarrow \infty$ in $C_{\varepsilon}$, and it is uniform with respect to $x_{0}$ when $x_{0}$ is confined to compact subsets of $\mathbb{R}$.

Remark 3.12. (i) In the special case $m=1$, the leading-order asymptotics (3.61) was published by Everitt, Hinton, and Shaw [33] in 1983. For asymptotic estimates of Weyl solutions in the case $m=1$, we refer to [98].

(ii) A comparison of (3.61) with (2.61) then proves that the leading-order asymptotic behavior (3.61) is in fact independent of the boundary condition at $x_{0}$ indexed by $\alpha$, that is,

$$
M_{ \pm}\left(z, x_{0}, \alpha\right) \underset{\substack{|z| \rightarrow \infty \\ z \in C_{\varepsilon}}}{=} \pm i I_{m}+o(1)
$$

for any $\alpha$ satisfying the conditions stated in (2.9). In the scalar case $m=1$, this fact had been noticed in [33. This boundary condition independence of the leadingorder asymptotic behavior of $M_{ \pm}\left(z, x_{0}, \alpha\right)$ is in sharp contrast to the case of matrixvalued Schrödinger operators (see, e.g., [20]). Moreover, regarding the conclusion of Theorem 3.11 no generality is lost by assuming that $C_{\varepsilon} \subset \mathbb{C}_{+}$, because of (2.58).

\section{Higher-Order Terms in the Asymptotic Expansion of $M_{ \pm}(z, x, \alpha)$}

In this section we shall prove one of our principal results of this paper, the asymptotic high-energy expansion of $M_{+}\left(z, x, \alpha_{0}\right)$ to arbitrarily high orders in sectors of the type $C_{\varepsilon} \subset \mathbb{C}_{+}$as defined in Theorem 3.11.

Throughout this section we choose $z \in \mathbb{C}_{+}$. We also recall the following notion: $x \in[a, b)$ (resp., $x \in(a, b])$ is called a right (resp., left) Lebesgue point of an element $q \in L^{1}((a, b)), a<b$, if $\int_{0}^{\varepsilon} d x^{\prime}\left|q\left(x+x^{\prime}\right)-q(x)\right|=o(\varepsilon)$ (resp., $\int_{0}^{\varepsilon} d x^{\prime}\left|q\left(x-x^{\prime}\right)-q(x)\right|$ $=o(\varepsilon))$ as $\varepsilon \downarrow 0$. Similarly, $x \in(a, b)$ is called a Lebesgue point of $q \in L^{1}((a, b))$ if $\int_{-\varepsilon}^{\varepsilon} d x^{\prime}\left|q\left(x+x^{\prime}\right)-q(x)\right|=o(\varepsilon)$ as $\varepsilon \downarrow 0$. The set of all such points is then called the right (resp., left) Lebesgue set of $q$ on $[a, b]$ in the former case and simply the Lebesgue set of $q$ on $[a, b]$ in the latter case. The analogous notions are applied to $2 m \times 2 m$ matrices $B \in L^{1}((a, b))^{2 m \times 2 m}$ by simultaneously considering all $4 m^{2}$ entries of $B$. The right (resp., left) Lebesgue set of $B$ on $[a, b]$ is then simply the intersection of the right (resp., left) Lebesgue sets of $B_{j, k}$ for all $1 \leq j, k \leq 2 m$, and similarly for the Lebesgue set of $B$, etc. 
Finally, we need one more ingredient, recently proven by Rybkin [102, Lemma 3] using appropriate maximal functions. Let $q \in L^{1}\left(\left(x_{0}, \infty\right)\right), \operatorname{supp}(q) \subseteq\left[x_{0}, x_{0}+R\right]$ for some $R>0$, and suppose $x \in\left[x_{0}, x_{0}+R\right]$ is a right Lebesgue point of $q$. Then

$$
\int_{x}^{x_{0}+R} d x^{\prime} q\left(x^{\prime}\right) \exp \left(2 i z\left(x^{\prime}-x\right)\right) \underset{\substack{|z| \rightarrow \infty \\ z \in C_{\varepsilon}}}{=}-\frac{q(x)}{2 i z}+o\left(|z|^{-1}\right) .
$$

An alternative proof of (4.1) follows from [118, Theorem I.13], which implies

$$
\lim _{\substack{|z| \rightarrow \infty \\ z \in C_{\varepsilon}}} z^{-1} \int_{x}^{x_{0}+R} d x^{\prime}\left|q\left(x^{\prime}\right)-q(x)\right| \exp \left(2 i z\left(x^{\prime}-x\right)\right)=0
$$

for any right Lebesgue point $x$ of $q$.

We start with the simpler case where $B$ has compact support contained in some interval $\left[x_{0}, y_{0}\right]$. Below in (4.3) and in analogous formulas in this section, $\|\cdot\|_{\mathbb{C}^{\ell \times \ell}}$ denotes a norm in $\mathbb{C}^{\ell \times \ell}$.

Lemma 4.1. Fix $x_{0}, y_{0} \in \mathbb{R}$ with $y_{0}>x_{0}$ and let $x \geq x_{0}$. Suppose $A=I_{2 m}$, $B \in L^{1}\left(\left[x_{0}, x_{0}+R\right]\right)^{2 m \times 2 m}$ for all $R>0$, and $B=B^{*}$ a.e. on $\left(x_{0}, \infty\right)$. In addition, assume that $B$ has compact support contained in $\left[x_{0}, y_{0}\right]$, that $B^{(N-1)} \in$ $L^{1}\left(\left[x_{0}, y_{0}\right]\right)^{2 m \times 2 m}$ for some $N \in \mathbb{N}$, that $x$ is a right Lebesgue point of $B^{(N-1)}$, and that

$$
\begin{aligned}
& \underset{y \in\left[x_{0}, y_{0}\right]}{\operatorname{ess} \sup _{0}}\left\|\int_{y}^{y_{0}} d x^{\prime} B^{(N-1)}\left(x^{\prime}\right) \exp \left(2 i z\left(x^{\prime}-y\right)\right)+\frac{1}{2 i z} B^{(N-1)}(y)\right\|_{\mathbb{C}^{2 m \times 2 m}} \\
& \underset{\substack{|z| \rightarrow \infty \\
z \in C_{\varepsilon}}}{=} o\left(|z|^{-1}\right) \text {. }
\end{aligned}
$$

If $N=1$, suppose in addition $B_{k, k^{\prime}} B_{\ell, \ell^{\prime}} \in L^{1}\left(\left[x_{0}, y_{0}\right]\right)^{m \times m}$ for all $k, k^{\prime}, \ell, \ell^{\prime} \in$ $\{1,2\}$. Let $\alpha_{0}=\left(I_{m} 0\right) \in \mathbb{C}^{m \times 2 m}$ and denote by $M_{+}\left(z, x, \alpha_{0}\right), x \geq x_{0}$, the unique Weyl-Titchmarsh matrix associated with the half-line Dirac-type operator $D_{+}\left(\alpha_{0}\right)$ in (2.89). Then, as $|z| \rightarrow \infty$ in $C_{\varepsilon}, M_{+}\left(z, x, \alpha_{0}\right)$ has an asymptotic expansion of the form

$$
M_{+}\left(z, x, \alpha_{0}\right) \underset{\substack{|z| \rightarrow \rightarrow \infty \\ z \in C_{\varepsilon}}}{=} i I_{m}+\sum_{k=1}^{N} m_{+, k}\left(x, \alpha_{0}\right) z^{-k}+o\left(|z|^{-N}\right), \quad N \in \mathbb{N} .
$$

The expansion (4.4) is uniform with respect to $\arg (z)$ for $|z| \rightarrow \infty$ in $C_{\varepsilon}$ and uniform in $x$ as long as $x$ varies in compact subintervals of $\left[x_{0}, \infty\right)$ intersected with the right Lebesgue set of $B^{(N-1)}$. The expansion coefficients $m_{+, k}\left(x, \alpha_{0}\right)$ can be 
recursively computed from

$$
\begin{aligned}
& m_{+, 1}\left(x, \alpha_{0}\right)=-\frac{1}{2}\left(B_{1,2}(x)+B_{2,1}(x)\right)+\frac{i}{2}\left(B_{1,1}(x)-B_{2,2}(x)\right), \\
& m_{+, k+1}\left(x, \alpha_{0}\right)=\frac{i}{2}\left(m_{+, k}^{\prime}\left(x, \alpha_{0}\right)+\sum_{\ell=1}^{k} m_{+, \ell}\left(x, \alpha_{0}\right) m_{+, k+1-\ell}\left(x, \alpha_{0}\right)\right. \\
&+\sum_{\ell=0}^{k} m_{+, \ell}\left(x, \alpha_{0}\right) B_{2,2}(x) m_{+, k-\ell}\left(x, \alpha_{0}\right) \\
&\left.+B_{1,2}(x) m_{+, k}\left(x, \alpha_{0}\right)+m_{+, k}\left(x, \alpha_{0}\right) B_{2,1}(x)\right), \\
& 1 \leq k \leq N-1 .
\end{aligned}
$$

Proof. In the following let $z \in \mathbb{C}_{+}$and $x \geq x_{0}$. The existence of an expansion of the type (4.4) is shown as follows. First one considers a matrix Volterra integral equation of the type

$$
\widetilde{U}_{+}\left(z, x, \alpha_{0}\right)=\left(\begin{array}{c}
I_{m} \\
i I_{m}
\end{array}\right) \exp \left(i z\left(x-x_{0}\right)\right)+\int_{x}^{\infty} d x^{\prime} K\left(z, x, x^{\prime}\right) J B\left(x^{\prime}\right) \widetilde{U}_{+}\left(z, x^{\prime}, \alpha_{0}\right),
$$

where

$$
\widetilde{U}_{+}\left(z, x, \alpha_{0}\right)=\left(\begin{array}{c}
\widetilde{u}_{+, 1}\left(z, x, \alpha_{0}\right) \\
\widetilde{u}_{+, 2}\left(z, x, \alpha_{0}\right)
\end{array}\right) \in L^{2}\left(\left[x_{0}, \infty\right)\right)^{2 m \times m},
$$

and $K$ abbreviates the $2 m \times 2 m$ Volterra Green's kernel

$$
K\left(z, x, x^{\prime}\right)=\left(\begin{array}{cc}
\cos \left(z\left(x-x^{\prime}\right)\right) I_{m} & \sin \left(z\left(x-x^{\prime}\right)\right) I_{m} \\
-\sin \left(z\left(x-x^{\prime}\right)\right) I_{m} & \cos \left(z\left(x-x^{\prime}\right)\right) I_{m}
\end{array}\right) .
$$

Clearly, $\widetilde{U}_{+}\left(z, \cdot, \alpha_{0}\right)$ solves the Dirac-type system (2.2) and (2.4). In addition, it satisfies $\widetilde{U}_{+}\left(z, \cdot, \alpha_{0}\right) \in L^{2}\left(\left[x_{0}, \infty\right)\right)^{2 m \times 2 m}$. Thus, up to normalization, $\widetilde{U}_{+}\left(z, \cdot, \alpha_{0}\right)$ represents the Weyl solution associated with $B$ on the half-line $\left[x_{0}, \infty\right)$. Next, introducing

$$
\widetilde{V}_{+}\left(z, x, \alpha_{0}\right)=\left(\begin{array}{c}
\widetilde{v}_{+, 1}\left(z, x, \alpha_{0}\right) \\
\widetilde{v}_{+, 2}\left(z, x, \alpha_{0}\right)
\end{array}\right)=\widetilde{U}_{+}\left(z, x, \alpha_{0}\right) \exp \left(-i z\left(x-x_{0}\right)\right),
$$

one rewrites (4.6) in the form

$$
\widetilde{V}_{+}\left(z, x, \alpha_{0}\right)=\left(\begin{array}{c}
I_{m} \\
i I_{m}
\end{array}\right)+\int_{x}^{y_{0}} d x^{\prime} \tilde{K}\left(z, x, x^{\prime}\right) J B\left(x^{\prime}\right) \widetilde{V}_{+}\left(z, x^{\prime}, \alpha_{0}\right),
$$

where

$$
\widetilde{K}\left(z, x, x^{\prime}\right)=\frac{1}{2}\left(\begin{array}{cc}
\left(1+\exp \left(2 i z\left(x^{\prime}-x\right)\right)\right) I_{m} & -i\left(1-\exp \left(2 i z\left(x^{\prime}-x\right)\right)\right) I_{m} \\
i\left(1-\exp \left(2 i z\left(x^{\prime}-x\right)\right)\right) I_{m} & \left(1+\exp \left(2 i z\left(x^{\prime}-x\right)\right)\right) I_{m}
\end{array}\right) .
$$

Thus, one infers

$$
M_{+}\left(z, x, \alpha_{0}\right)=\widetilde{u}_{+, 2}\left(z, x, \alpha_{0}\right) \widetilde{u}_{+, 1}\left(z, x, \alpha_{0}\right)^{-1}=\widetilde{v}_{+, 2}\left(z, x, \alpha_{0}\right) \widetilde{v}_{+, 1}\left(z, x, \alpha_{0}\right)^{-1} .
$$


Introducing

$$
R=\left(\begin{array}{cc}
C_{1} & -i C_{2} \\
i C_{1} & C_{2}
\end{array}\right), \quad S=\left(\begin{array}{cc}
D_{1} & i D_{2} \\
-i D_{1} & D_{2}
\end{array}\right)
$$

where

$$
\begin{aligned}
& C_{1}=-B_{1,2}^{*}-i B_{1,1}, \quad C_{2}=B_{1,2}-i B_{2,2} \\
& D_{1}=-B_{1,2}^{*}+i B_{1,1}, \quad D_{2}=B_{1,2}+i B_{2,2}
\end{aligned}
$$

we get from 4.10)

$$
\begin{gathered}
\widetilde{V}_{+}\left(z, x, \alpha_{0}\right)=\left(\begin{array}{c}
I_{m} \\
i I_{m}
\end{array}\right)+\int_{x}^{y_{0}} d x^{\prime}\left(R\left(x^{\prime}\right)+S\left(x^{\prime}\right) \exp \left(2 i z\left(x^{\prime}-x\right)\right)\right) \widetilde{V}_{+}\left(z, x^{\prime}, \alpha_{0}\right) \\
=\left(I_{2 m}+\sum_{k=1}^{\infty} 2^{-k} \int_{x}^{y_{0}} d x_{1}\left(R\left(x_{1}\right)+S\left(x_{1}\right) e^{2 i z\left(x_{1}-x\right)}\right)\right. \\
\times \int_{x_{1}}^{y_{0}} d x_{2}\left(R\left(x_{2}\right)+S\left(x_{2}\right) e^{2 i z\left(x_{2}-x_{1}\right)}\right) \\
\left.\cdots \int_{x_{k-1}}^{y_{0}} d x_{k}\left(R\left(x_{k}\right)+S\left(x_{k}\right) e^{2 i z\left(x_{k}-x_{k-1}\right)}\right)\right)\left(\begin{array}{c}
I_{m} \\
i I_{m}
\end{array}\right) .
\end{gathered}
$$

This yields

$$
\left\|\widetilde{v}_{+, j}\left(z, x, \alpha_{0}\right)\right\| \leq C_{j}, \quad z \in \mathbb{C}_{+}, \operatorname{Im}(z)>0, x \geq x_{0}, j=1,2,
$$

for some $C_{j}>0, j=1,2$, depending on $\|B\|_{1}$. Integrating by parts in (4.17) and repeatedly applying (4.1) and (4.3) to $q(x)=(S(x))_{j, k}$ for all $1 \leq j, k \leq 2 m$ then results in the existence of an asymptotic expansion for $\widetilde{V}_{+}\left(z, x, \alpha_{0}\right)$ of the type

$$
\widetilde{V}_{+}\left(z, x, \alpha_{0}\right)=\left(\begin{array}{c}
\widetilde{v}_{+, 1}\left(z, x, \alpha_{0}\right) \\
\widetilde{v}_{+, 2}\left(z, x, \alpha_{0}\right)
\end{array}\right)=\sum_{k=0}^{N} \widetilde{V}_{+, k}\left(x, \alpha_{0}\right) z^{-k}+o\left(|z|^{-N}\right) .
$$

Inserting the expansions for $\widetilde{v}_{+, 2}\left(z, x, \alpha_{0}\right)$ and $\widetilde{v}_{+, 1}\left(z, x, \alpha_{0}\right)^{-1}$ into (4.12) (using a geometric series expansion for $\left.\widetilde{v}_{+, 1}\left(z, x, \alpha_{0}\right)^{-1}\right)$ then yields the existence of an expansion of the type (4.4) for $M_{+}\left(z, x, \alpha_{0}\right)$. The actual expansion coefficients and the associated recursion relation (4.5) then follow upon inserting expansion (4.4) into the Riccati-type equation (3.16a). The stated uniformity assertions concerning the asymptotic expansion (4.4) then follow from iterating the system of Volterra integral equations [4.10).

Remark 4.2. The analogous solution $\widetilde{U}_{-}\left(z, \cdot, \alpha_{0}\right)$ of the Dirac-type operator (2.76) on the interval $\left(-\infty, x_{0}\right]$ satisfies

$$
\begin{aligned}
\widetilde{U}_{-}\left(z, x, \alpha_{0}\right)= & \left(\begin{array}{c}
I_{m} \\
-i I_{m}
\end{array}\right) \exp \left(-i z\left(x-x_{0}\right)\right) \\
& -\int_{-\infty}^{x} d x^{\prime} K\left(z, x, x^{\prime}\right) J \widetilde{B}\left(x^{\prime}\right) \widetilde{U}_{-}\left(z, x^{\prime}, \alpha_{0}\right),
\end{aligned}
$$

with integral kernel $K$ given by (4.8). (Again $\widetilde{U}_{-}$coincides with the Weyl solution $U_{-}$up to normalization.) A closer look at the system of Volterra integral equations 
(4.6), (4.16), (4.17), and similarly in connection with (4.20), then reveals that $\widetilde{U}_{ \pm}\left(z, \cdot, \alpha_{0}\right)$ have the asymptotic behavior

$$
\widetilde{U}_{ \pm}\left(z, x, \alpha_{0}\right) \underset{\substack{|z| \rightarrow \infty \\
z \in C_{\varepsilon}}}{=}\left(\sum_{k=0}^{N}\left(\begin{array}{l}
\widetilde{v}_{ \pm, k, 1}\left(x, \alpha_{0}\right) \\
\widetilde{v}_{ \pm, k, 2}\left(x, \alpha_{0}\right)
\end{array}\right) z^{-k}+o\left(|z|^{-N}\right)\right) \exp \left( \pm i z\left(x-x_{0}\right)\right),
$$

with leading asymptotics determined as follows:

$$
\begin{aligned}
& \widetilde{v}_{ \pm, 0,1}\left(x, \alpha_{0}\right)=I_{m}+\widetilde{w}_{ \pm, 0,1}\left(x, \alpha_{0}\right), \\
& \widetilde{v}_{ \pm, 0,2}\left(x, \alpha_{0}\right)= \pm i\left(I_{m}+\widetilde{w}_{ \pm, 0,1}\left(x, \alpha_{0}\right)\right),
\end{aligned}
$$

where $\widetilde{w}_{ \pm, 0,1}\left(x, \alpha_{0}\right)$ satisfies

$$
\widetilde{w}_{ \pm, 0,1}^{\prime}\left(x, \alpha_{0}\right)=\frac{1}{2}\left[\widetilde{B}_{2,1}(x)-\widetilde{B}_{1,2}(x) \pm i \widetilde{B}_{2,2}(x) \pm i \widetilde{B}_{1,1}(x)\right]\left(I_{m}+\widetilde{w}_{ \pm, 0,1}\left(x, \alpha_{0}\right)\right)
$$

and

$$
\lim _{x \rightarrow \pm \infty} \widetilde{w}_{ \pm, 0,1}\left(x, \alpha_{0}\right)=0
$$

(in fact, $\widetilde{v}_{ \pm, 0,1}\left(\cdot, \alpha_{0}\right)=I_{m}, \widetilde{v}_{ \pm, 0,2}\left(\cdot, \alpha_{0}\right)= \pm i I_{m}$, and $\widetilde{v}_{ \pm, k, j}\left(\cdot, \alpha_{0}\right)=0, j=1,2$, $1 \leq k \leq N$, outside the support of $\widetilde{B})$. In particular,

$$
\widetilde{w}_{ \pm, 0,1}\left(x, \alpha_{0}\right)=0 \text {, }
$$

and hence

$$
\widetilde{U}_{ \pm}\left(z, x, \alpha_{0}\right) \underset{\substack{|z| \rightarrow \infty \\
z \in C_{\varepsilon}}}{=}\left(\left(\begin{array}{c}
I_{m} \\
\pm i I_{m}
\end{array}\right)+o(1)\right) \exp \left( \pm i z\left(x-x_{0}\right)\right)
$$

if and only if $\widetilde{B}$ is in the normal form

$$
\widetilde{B}(x)=\left(\begin{array}{cc}
\widetilde{B}_{1,1}(x) & \widetilde{B}_{1,2}(x) \\
\widetilde{B}_{1,2}(x) & -\widetilde{B}_{1,1}(x)
\end{array}\right), \quad \widetilde{B}_{1,1}^{*}(x)=\widetilde{B}_{1,1}(x), \widetilde{B}_{1,2}^{*}(x)=\widetilde{B}_{1,2}(x) \text { a.e. }
$$

For more details we refer to Lemma 5.1 .

Next we recall an elementary result on finite-dimensional evolution equations essentially taken from [25, p. 103] (cf. also [20 Lemma 4.2]).

Lemma 4.3 ([25, p. 103]). Let $\Gamma_{j} \in L_{\mathrm{loc}}^{1}(\mathbb{R})^{m \times m}, j=1,2$. Then any $m \times m$ matrix-valued solution $X$ of

$$
X^{\prime}(x)=\Gamma_{1}(x) X(x)+X(x) \Gamma_{2}(x) \text { for a.e. } x \in \mathbb{R},
$$

is of the type

$$
X(x)=Y(x) C Z(x),
$$

where $C$ is a constant $m \times m$ matrix and $Y$ is a fundamental system of solutions of

$$
\Psi^{\prime}(x)=\Gamma_{1}(x) \Psi(x)
$$

and $Z$ is a fundamental system of solutions of

$$
\Phi^{\prime}(x)=\Phi(x) \Gamma_{2}(x) .
$$


The next result provides the proper extension of Lemma 4.3 in [20] in the context of matrix-valued Schrödinger operators (which in turn extended Proposition 2.1 in the scalar context in [50] to the matrix-valued case) to the Dirac-type case under consideration.

Lemma 4.4. Fix $x_{0}, y_{0} \in \mathbb{R}$ with $y_{0}>x_{0}$. Suppose that $A_{j}=I_{2 m}$, and that $B_{j} \in L^{1}\left(\left[x_{0}, x_{0}+R\right]\right)^{2 m \times 2 m}$ for all $R>0, B_{j}=B_{j}^{*}$ a.e. on $\left[x_{0}, \infty\right), j=1,2$, and $B_{1}=B_{2}$ a.e. on $\left[x_{0}, y_{0}\right]$. Let $\alpha_{0}=\left(I_{m} 0\right) \in \mathbb{C}^{m \times 2 m}$ and denote by $M_{j,+}\left(z, x, \alpha_{0}\right)$, $x \geq x_{0}$, the unique Weyl-Titchmarsh matrix corresponding to the half-line Dirac operators $D_{+, j}\left(\alpha_{0}\right), j=1,2$, in (2.89). Then,

$$
\begin{aligned}
& {\left[M_{1,+}^{\prime}\left(z, x, \alpha_{0}\right)-M_{2,+}^{\prime}\left(z, x, \alpha_{0}\right)\right] } \\
&=-(z / 2)\left[M_{1,+}\left(z, x, \alpha_{0}\right)+M_{2,+}\left(z, x, \alpha_{0}\right)\right]\left[M_{1,+}\left(z, x, \alpha_{0}\right)-M_{2,+}\left(z, x, \alpha_{0}\right)\right] \\
&-(z / 2)\left[M_{1,+}\left(z, x, \alpha_{0}\right)-M_{2,+}\left(z, x, \alpha_{0}\right)\right]\left[M_{1,+}\left(z, x, \alpha_{0}\right)+M_{2,+}\left(z, x, \alpha_{0}\right)\right] \\
&-\left[M_{1,+}\left(z, x, \alpha_{0}\right)+M_{2,+}\left(z, x, \alpha_{0}\right)\right] B_{2,2}(x)\left[M_{1,+}\left(z, x, \alpha_{0}\right)-M_{2,+}\left(z, x, \alpha_{0}\right)\right] / 2 \\
&-\left[M_{1,+}\left(z, x, \alpha_{0}\right)-M_{2,+}\left(z, x, \alpha_{0}\right)\right] B_{2,2}(x)\left[M_{1,+}\left(z, x, \alpha_{0}\right)+M_{2,+}\left(z, x, \alpha_{0}\right)\right] / 2 \\
&-B_{1,2}(x)\left[M_{1,+}\left(z, x, \alpha_{0}\right)-M_{2,+}\left(z, x, \alpha_{0}\right)\right] \\
&-\left[M_{1,+}\left(z, x, \alpha_{0}\right)-M_{2,+}\left(z, x, \alpha_{0}\right)\right] B_{2,1}(x) \text { for a.e. } x \in\left[x_{0}, y_{0}\right],
\end{aligned}
$$

where we denoted $B_{1}=B_{2}=\left(\begin{array}{ll}B_{1,1} & B_{1,2} \\ B_{2,1} & B_{2,2}\end{array}\right)$ a.e. on $\left(x_{0}, y_{0}\right)$.

Proof. This is obvious from (3.16a).

Lemma 4.5. Fix $x_{0}, y_{0} \in \mathbb{R}$ with $y_{0}>x_{0}$. Suppose that $A_{j}=I_{2 m}$, and that $B_{j} \in L^{1}\left(\left[x_{0}, x_{0}+R\right]\right)^{2 m \times 2 m}$ for all $R>0$, and $B_{j}=B_{j}^{*}$ a.e. on $\left[x_{0}, \infty\right), j=1,2$. Let $\alpha_{0}=\left(I_{m} 0\right) \in \mathbb{C}^{m \times 2 m}$ and denote by $M_{j,+}\left(z, x, \alpha_{0}\right), x \geq x_{0}$, the unique Weyl-Titchmarsh matrix corresponding to the half-line Dirac operators $D_{+, j}\left(\alpha_{0}\right)$, $j=1,2$, in (2.89). Define

$$
\begin{aligned}
\Gamma_{1}(z, x)= & -(z / 2)\left[M_{1,+}\left(z, x, \alpha_{0}\right)+M_{2,+}\left(z, x, \alpha_{0}\right)\right] \\
& -(1 / 2)\left[M_{1,+}\left(z, x, \alpha_{0}\right)+M_{2,+}\left(z, x, \alpha_{0}\right)\right] B_{2,2}(x)-B_{1,2}(x), \\
\Gamma_{2}(z, x)= & -(z / 2)\left[M_{1,+}\left(z, x, \alpha_{0}\right)+M_{2,+}\left(z, x, \alpha_{0}\right)\right] \\
& -(1 / 2) B_{2,2}(x)\left[M_{1,+}\left(z, x, \alpha_{0}\right)+M_{2,+}\left(z, x, \alpha_{0}\right)\right]-B_{2,1}(x),
\end{aligned}
$$

for a.e. $x \in\left[x_{0}, y_{0}\right]$. In addition, assume $Y_{+}(z, \cdot)$ and $Z_{+}(z, \cdot)$ to be fundamental matrix solutions of

$$
\Psi^{\prime}(z, x)=\Gamma_{1}(z, x) \Psi(z, x) \quad \text { and } \quad \Phi^{\prime}(z, x)=\Phi(z, x) \Gamma_{2}(z, x)
$$

on $\left[x_{0}, y_{0}\right]$, respectively, with

$$
Y_{+}\left(z, y_{0}\right)=I_{m}, \quad Z_{+}\left(z, y_{0}\right)=I_{m} .
$$

Then, as $|z| \rightarrow \infty, z \in C_{\varepsilon}$,

$$
\left\|Y_{+}\left(z, x_{0}\right)\right\|_{\mathbb{C}^{m \times m}},\left\|Z_{+}\left(z, x_{0}\right)\right\|_{\mathbb{C}^{m \times m}} \leq \exp \left(-\operatorname{Im}(z)\left(y_{0}-x_{0}\right)(1+o(1))\right) .
$$

Proof. Define $\widetilde{\Gamma}_{j}(z, x), j=1,2$, by

$$
\widetilde{\Gamma}_{j}(z, x)=\Gamma_{j}(z, x)+i z I_{m}, \quad j=1,2 ;
$$


then

$$
\int_{x_{0}}^{y_{0}} d x\left\|\widetilde{\Gamma}_{j}(z, x)\right\|_{\mathbb{C}^{m \times m}} \underset{\substack{|z| \rightarrow \infty \\ z \in C_{\varepsilon}}}{=} o(z), \quad j=1,2,
$$

due to the uniform nature of the asymptotic expansion (3.61) for $x$ varying in compact intervals. Next, introduce

$$
E_{+}\left(z, x, y_{0}\right)=I_{m} \exp \left(i z\left(y_{0}-x\right)\right), \quad x \leq y_{0} ;
$$

then

$$
\begin{aligned}
& Y_{+}(z, x)=E_{+}\left(z, x, y_{0}\right)-\int_{x}^{y_{0}} d x^{\prime} E_{+}\left(z, x, x^{\prime}\right) \widetilde{\Gamma}_{1}\left(z, x^{\prime}\right) Y_{+}\left(z, x^{\prime}\right), \\
& Z_{+}(z, x)=E_{+}\left(z, x, y_{0}\right)-\int_{x}^{y_{0}} d x^{\prime} Z_{+}\left(z, x^{\prime}\right) \widetilde{\Gamma}_{2}\left(z, x^{\prime}\right) E_{+}\left(z, x, x^{\prime}\right) .
\end{aligned}
$$

Using

$$
\left\|E_{+}\left(z, x_{0}, y_{0}\right)\right\|_{\mathbb{C}^{m \times m}} \leq \exp \left(-\operatorname{Im}(z)\left(y_{0}-x_{0}\right)\right),
$$

a standard Volterra-type iteration argument in 4.41), (4.42) then yields

$$
\begin{aligned}
&\left\|Y_{+}\left(z, x_{0}\right)\right\|_{\mathbb{C}^{m \times m}} \leq \exp \left(-\operatorname{Im}(z)\left(y_{0}-x_{0}\right)+\int_{x_{0}}^{y_{0}} d x\left\|\widetilde{\Gamma}_{1}(z, x)\right\|\right), \\
&\left\|Z_{+}\left(z, x_{0}\right)\right\|_{\mathbb{C}^{m \times m}} \leq \exp \left(-\operatorname{Im}(z)\left(y_{0}-x_{0}\right)+\int_{x_{0}}^{y_{0}} d x\left\|\widetilde{\Gamma}_{2}(z, x)\right\|\right),
\end{aligned}
$$

and hence (4.37).

Theorem 4.6. Fix $x_{0}, y_{0} \in \mathbb{R}$ with $y_{0}>x_{0}$. Suppose that $A_{j}=I_{2 m}$, and that $B_{j} \in L^{1}\left(\left[x_{0}, x_{0}+R\right]\right)^{2 m \times 2 m}$ for all $R>0, B_{j}=B_{j}^{*}$ a.e. on $\left[x_{0}, \infty\right), j=1,2$, and $B_{1}=B_{2}$ a.e. on $\left[x_{0}, y_{0}\right]$. Let $\alpha_{0}=\left(I_{m} 0\right) \in \mathbb{C}^{m \times 2 m}$ and denote by $M_{j,+}\left(z, x, \alpha_{0}\right)$, $x \geq x_{0}$, the unique Weyl-Titchmarsh matrix corresponding to the half-line Dirac operators $D_{+, j}\left(\alpha_{0}\right), j=1,2$, in (2.89). Then, as $|z| \rightarrow \infty$ in $C_{\varepsilon}$,

$$
\left\|M_{1,+}\left(z, x_{0}, \alpha_{0}\right)-M_{2,+}\left(z, x_{0}, \alpha_{0}\right)\right\|_{\mathbb{C}^{m \times m}} \leq C \exp \left(-2 \operatorname{Im}(z)\left(y_{0}-x_{0}\right)(1+o(1))\right)
$$

for some constant $C>0$.

Proof. Define for $z \in \mathbb{C} \backslash \mathbb{R}, x \in\left[x_{0}, y_{0}\right]$,

$$
X_{+}(z, x)=M_{1,+}\left(z, x, \alpha_{0}\right)-M_{2,+}\left(z, x, \alpha_{0}\right),
$$

and for $z \in \mathbb{C} \backslash \mathbb{R}$ and a.e. $x \in\left[x_{0}, y_{0}\right]$,

$$
\begin{aligned}
\Gamma_{1}(z, x)= & -(z / 2)\left[M_{1,+}\left(z, x_{0}, \alpha_{0}\right)+M_{2,+}\left(z, x_{0}, \alpha_{0}\right)\right] \\
& -(1 / 2)\left[M_{1,+}\left(z, x_{0}, \alpha_{0}\right)+M_{2,+}\left(z, x_{0}, \alpha_{0}\right)\right] B_{2,2}(x)-B_{1,2}(x), \\
\Gamma_{2}(z, x)= & -(z / 2)\left[M_{1,+}\left(z, x_{0}, \alpha_{0}\right)+M_{2,+}\left(z, x_{0}, \alpha_{0}\right)\right] \\
& -(1 / 2) B_{2,2}(x)\left[M_{1,+}\left(z, x_{0}, \alpha_{0}\right)+M_{2,+}\left(z, x_{0}, \alpha_{0}\right)\right]-B_{2,1}(x) .
\end{aligned}
$$

$$
X_{+}^{\prime}=\Gamma_{1} X_{+}+X_{+} \Gamma_{2}
$$

and hence, by Lemma 4.3 .

$$
X_{+}(z, x)=Y_{+}(z, x) X_{+}\left(z, x_{1}\right) Z_{+}(z, x),
$$


where $Y_{+}(z, x)$ and $Z_{+}(z, x)$ are fundamental solution matrices of

$$
\Psi^{\prime}(z, x)=\Gamma_{1}(z, x) \Psi(z, x) \quad \text { and } \quad \Phi^{\prime}(z, x)=\Phi(z, x) \Gamma_{2}(z, x),
$$

respectively, with

$$
Y_{+}\left(z, y_{0}\right)=I_{m}, \quad Z_{+}\left(z, y_{0}\right)=I_{m}
$$

By Lemma 4.5

$$
\left.\left\|Y_{+}\left(z, x_{0}\right)\right\|_{\mathbb{C}^{m \times m}},\left\|Z_{+}\left(z, x_{0}\right)\right\|_{\mathbb{C}^{m \times m}} \leq \exp \left(-\operatorname{Im}(z)\left(y_{0}-x_{0}\right)\right)(1+o(1))\right)
$$

as $|z| \rightarrow \infty, z \in C_{\varepsilon}$. Thus, as $|z| \rightarrow \infty, z \in C_{\varepsilon}$,

$$
\begin{aligned}
\left\|X_{+}\left(z, x_{0}\right)\right\|_{\mathbb{C}^{m \times m}} & \leq\left\|X_{+}\left(z, y_{0}\right)\right\|_{\mathbb{C}^{m \times m}}\left\|Y_{+}\left(z, x_{0}\right)\right\|_{\mathbb{C}^{m \times m}}\left\|Z_{+}\left(z, x_{0}\right)\right\|_{\mathbb{C}^{m \times m}} \\
& \leq C \exp \left(-2 \operatorname{Im}(z)\left(y_{0}-x_{0}\right)(1+o(1))\right)
\end{aligned}
$$

for some constant $C>0$ by (3.61), (4.51), and (4.54).

Given these preparations, we can now drop the compact support assumption on $B$ in Lemma 4.1 and hence arrive at one of the principal results of this paper.

Theorem 4.7. Fix $x_{0}, y_{0} \in \mathbb{R}$ with $y_{0}>x_{0}$, and suppose that $A=I_{2 m}$, and that $B \in L^{1}\left(\left[x_{0}, x_{0}+R\right]\right)^{2 m \times 2 m}$ for all $R>0$, and $B=B^{*}$ a.e. on $\left(x_{0}, \infty\right)$. In addition, assume that for some $N \in \mathbb{N}, B^{(N-1)} \in L^{1}\left(\left[x_{0}, c\right]\right)^{2 m \times 2 m}$ for all $c>x_{0}$, that $x_{0}$ is a right Lebesgue point of $B^{(N-1)}$, and that

$$
\begin{aligned}
& \underset{\substack {\left.\operatorname{ess} \sup _{0}, y_{0}\right] \\
\begin{subarray}{c}{|z| \rightarrow \infty \\
z \in C_{\varepsilon}{ \operatorname { e s s } \operatorname { s u p } _ { 0 } , y _ { 0 } ] \\
\begin{subarray} { c } { | z | \rightarrow \infty \\
z \in C _ { \varepsilon } } }\end{subarray}}{=}\left(\mid \int_{y}^{y_{0}} d x^{\prime} B^{(N-1)}\left(x^{\prime}\right) \exp \left(2 i z\left(x^{\prime}-y\right)\right)+\frac{1}{2 i z} B^{(N-1)}(y) \|_{\mathbb{C}^{2 m \times 2 m}}\right. \\
&
\end{aligned}
$$

If $N=1$, suppose in addition $B_{k, k^{\prime}} B_{\ell, \ell^{\prime}} \in L^{1}\left(\left[x_{0}, y_{0}\right]\right)^{m \times m}$ for all $k, k^{\prime}, \ell, \ell^{\prime} \in$ $\{1,2\}$. Let $\alpha_{0}=\left(I_{m} 0\right) \in \mathbb{C}^{m \times 2 m}$ and denote by $M_{+}\left(z, x_{0}, \alpha_{0}\right)$ the unique element of the limit disk $\mathcal{D}_{+}\left(z, x_{0}, \alpha_{0}\right)$ for the half-line Dirac operator $D_{+}\left(\alpha_{0}\right)$ in (2.89). Then, as $|z| \rightarrow \infty$ in $C_{\varepsilon}, M_{+}\left(z, x_{0}, \alpha_{0}\right)$ has an asymptotic expansion of the form

$$
M_{+}\left(z, x_{0}, \alpha_{0}\right) \underset{\substack{|z| \rightarrow \infty \\ z \in C_{\varepsilon}}}{=} i I_{m}+\sum_{k=1}^{N} m_{+, k}\left(x_{0}, \alpha_{0}\right) z^{-k}+o\left(|z|^{-N}\right), \quad N \in \mathbb{N} .
$$

The expansion (4.57) is uniform with respect to $\arg (z)$ for $|z| \rightarrow \infty$ in $C_{\varepsilon}$. The expansion coefficients $m_{+, k}\left(x_{0}, \alpha_{0}\right)$ can be recursively computed from (4.5).

Proof. Define

$$
\widetilde{B}(x)= \begin{cases}B(x) & \text { for } x \in\left[x_{0}, y_{0}\right], x_{0}<y_{0}, \\ 0 & \text { otherwise }\end{cases}
$$

and apply Theorem 4.6 with $B_{1}=B, B_{2}=\widetilde{B}$. Then (in obvious notation)

$$
\left\|M_{+}\left(z, x_{0}, \alpha_{0}\right)-\widetilde{M}_{+}\left(z, x_{0}, \alpha_{0}\right)\right\|_{\mathbb{C}^{m \times m}} \leq C \exp \left(-2 \operatorname{Im}(z)\left(y_{0}-x_{0}\right)(1+o(1))\right)
$$

as $|z| \rightarrow \infty, z \in C_{\varepsilon}$, and hence the asymptotic expansion (4.4) for $\widetilde{M}_{+}\left(z, x_{0}, \alpha_{0}\right)$ in Lemma 4.1 coincides with that of $M_{+}\left(z, x_{0}, \alpha_{0}\right)$. 
In analogy to Theorem [3.11, the asymptotic expansion (4.57) extends to one for $M_{+}\left(z, x, \alpha_{0}\right)$ valid uniformly with respect to $x$ as long as $x$ varies in compact subintervals of $\left[x_{0}, \infty\right)$ intersected with the right Lebesgue set of $B^{(N-1)}$.

Theorem 4.8. Fix $x_{0} \in \mathbb{R}$ and let $x \geq x_{0}$. Suppose that $A=I_{2 m}$, and that $B \in L^{1}\left(\left[x_{0}, x_{0}+R\right]\right)^{2 m \times 2 m}$ for all $R>0$, and $B=B^{*}$ a.e. on $\left(x_{0}, \infty\right)$. In addition, assume that for some $N \in \mathbb{N}, B^{(N-1)} \in L^{1}\left(\left[x_{0}, c\right)\right)^{2 m \times 2 m}$ for all $c>x_{0}$, that $x$ is a right Lebesgue point of $B^{(N-1)}$, and that for all $R>0$,

$$
\operatorname{ess~sup}_{y \in\left[x_{0}, x_{0}+R\right]}\left\|\int_{y}^{x_{0}+R} d x^{\prime} B^{(N-1)}\left(x^{\prime}\right) \exp \left(2 i z\left(x^{\prime}-y\right)\right)+\frac{1}{2 i z} B^{(N-1)}(y)\right\|_{\mathbb{C}^{2 m \times 2 m}}
$$

$$
\underset{\substack{|z| \rightarrow \infty \\ z \in C_{\varepsilon}}}{=} o\left(|z|^{-1}\right)
$$

If $N=1$, suppose in addition $B_{k, k^{\prime}} B_{\ell, \ell^{\prime}} \in L^{1}\left(\left[x_{0}, x_{0}+R\right]\right)^{m \times m}$ for all $R>0$ and all $k, k^{\prime}, \ell, \ell^{\prime} \in\{1,2\}$. Let $\alpha_{0}=\left(I_{m} 0\right) \in \mathbb{C}^{m \times 2 m}$ and denote by $M_{+}\left(z, x, \alpha_{0}\right)$, $x \geq x_{0}$, the unique element of the limit disk $\mathcal{D}_{+}\left(z, x, \alpha_{0}\right)$ for the half-line Dirac operator $D_{+}\left(\alpha_{0}\right)$ in (2.89). Then, as $|z| \rightarrow \infty$ in $C_{\varepsilon}, M_{+}\left(z, x, \alpha_{0}\right)$ has an asymptotic expansion of the form

$$
M_{+}\left(z, x, \alpha_{0}\right) \underset{\substack{|z| \rightarrow \infty \\ z \in C_{\varepsilon}}}{=} i I_{m}+\sum_{k=1}^{N} m_{+, k}\left(x, \alpha_{0}\right) z^{-k}+o\left(|z|^{-N}\right), \quad N \in \mathbb{N}
$$

The expansion (4.61) is uniform with respect to $\arg (z)$ for $|z| \rightarrow \infty$ in $C_{\varepsilon}$, and uniform in $x$ as long as $x$ varies in compact subsets of $\mathbb{R}$ intersected with the right Lebesgue set of $B^{(N-1)}$. The expansion coefficients $m_{+, k}\left(x, \alpha_{0}\right)$ can be recursively computed from (4.5).

Proof. To see that uniformity holds for this expansion, first recall the role of Theorem 3.11 in providing uniformity in the asymptotic expression (4.39), which then leads to (4.37) holding uniformly with respect to $x_{0}$ varying within compact subsets of $\mathbb{R}$ and with respect to $\arg (z)$ for $|z| \rightarrow \infty$ in $C_{\varepsilon}$. This in turn leads to a similar uniformity holding for (4.46), which is the key to (4.57) holding with respect to $x_{0}$ varying within compact subsets of $\mathbb{R}$ and with respect to $\arg (z)$ for $|z| \rightarrow \infty$ in $C_{\varepsilon}$.

Remark 4.9. For simplicity, we have focused on the expansion of $M_{+}\left(z, x_{0}, \alpha_{0}\right)$ as $|z| \rightarrow \infty$. Of course, Theorem 4.8 holds also for $M_{-}\left(z, x_{0}, \alpha_{0}\right)$ if we replace the hypotheses concerning right Lebesgue points by those for left Lebesgue points, etc. For convenience we just state the corresponding expansion and associated nonlinear recursion formula which covers both cases:

$$
M_{ \pm}\left(z, x, \alpha_{0}\right) \underset{\substack{|z| \rightarrow \infty \\ z \in C_{\varepsilon}}}{=} \sum_{k=0}^{N} m_{ \pm, k}\left(x, \alpha_{0}\right) z^{-k}+o\left(|z|^{-N}\right), \quad N \in \mathbb{N}
$$




$$
\begin{aligned}
& m_{ \pm, 0}\left(x, \alpha_{0}\right)= \pm i I_{m} \\
& m_{ \pm, 1}\left(x, \alpha_{0}\right)=-\frac{1}{2}\left(B_{1,2}(x)+B_{2,1}(x)\right) \pm \frac{i}{2}\left(B_{1,1}(x)-B_{2,2}(x)\right), \\
& m_{ \pm, k+1}\left(x, \alpha_{0}\right)= \pm \frac{i}{2}\left(m_{ \pm, k}^{\prime}\left(x, \alpha_{0}\right)+\sum_{\ell=1}^{k} m_{ \pm, \ell}\left(x, \alpha_{0}\right) m_{ \pm, k+1-\ell}\left(x, \alpha_{0}\right)\right. \\
& \quad+\sum_{\ell=0}^{k} m_{ \pm, \ell}\left(x, \alpha_{0}\right) B_{2,2}(x) m_{ \pm, k-\ell}\left(x, \alpha_{0}\right) \\
&\left.+B_{1,2}(x) m_{ \pm, k}\left(x, \alpha_{0}\right)+m_{ \pm, k}\left(x, \alpha_{0}\right) B_{2,1}(x)\right), \\
& 1 \leq k \leq N-1 .
\end{aligned}
$$

Combining Theorem 4.8 and $(2.78)$ then yields the analogous asymptotic expansion for $M\left(z, x, \alpha_{0}\right)$.

Theorem 4.10. Assume Hypothesis 2.1 with $A=I_{2 m}$, and let $\alpha_{0}=\left(I_{m} 0\right) \in$ $\mathbb{C}^{m \times 2 m}$. Fix $x_{0} \in \mathbb{R}$ and let $x \in \mathbb{R}$. Suppose that for some $N \in \mathbb{N}, B^{(N-1)} \in$ $L_{\text {loc }}^{1}(\mathbb{R})^{2 m \times 2 m}$, that $x$ is a right and a left Lebesgue point of $B^{(N-1)}$, and that for all $R>0$,

$$
\begin{aligned}
& \operatorname{esssup}_{y \in\left[x_{0}, x_{0}+R\right]}\left\|\int_{y}^{x_{0}+R} d x^{\prime} B^{(N-1)}\left(x^{\prime}\right) \exp \left(2 i z\left(x^{\prime}-y\right)\right)+\frac{1}{2 i z} B^{(N-1)}(y)\right\|_{\mathbb{C}^{2 m \times 2 m}} \\
& +\underset{y \in\left[x_{0}-R, x_{0}\right]}{\operatorname{essup}}\left\|\int_{x_{0}-R}^{y} d x^{\prime} B^{(N-1)}\left(x^{\prime}\right) \exp \left(2 i z\left(x^{\prime}-y\right)\right)-\frac{1}{2 i z} B^{(N-1)}(y)\right\|_{\mathbb{C}^{2 m \times 2 m}}
\end{aligned}
$$

$$
\underset{\substack{|z| \rightarrow \infty \\ z \in C_{\varepsilon}}}{=} o\left(|z|^{-1}\right)
$$

If $N=1$, assume in addition $B_{k, k^{\prime}} B_{\ell, \ell^{\prime}} \in L_{\mathrm{loc}}^{1}(\mathbb{R})^{m \times m}$ for all $k, k^{\prime}, \ell, \ell^{\prime} \in\{1,2\}$. Let $M\left(z, x, \alpha_{0}\right)$ be defined as in (2.77) (see also (2.78)). Then, as $|z| \rightarrow \infty$ in $C_{\varepsilon}$, $M\left(z, x, \alpha_{0}\right)$ has an asymptotic expansion of the form

$$
M\left(z, x, \alpha_{0}\right) \underset{\substack{|z| \rightarrow \infty \\ z \in C_{\varepsilon}}}{=}(i / 2) I_{2 m}+\sum_{k=1}^{N} M_{k}\left(x, \alpha_{0}\right) z^{-k}+o\left(|z|^{-N}\right), \quad N \in \mathbb{N},
$$

where

$$
\begin{aligned}
& M_{1}\left(x, \alpha_{0}\right)=-\frac{i}{8}\left(\begin{array}{ll}
B_{1,1}(x+0)-B_{2,2}(x+0) & B_{1,2}(x+0)+B_{2,1}(x+0) \\
B_{1,2}(x+0)+B_{2,1}(x+0) & B_{2,2}(x+0)-B_{1,1}(x+0)
\end{array}\right) \\
&(4.66) \quad-\frac{i}{8}\left(\begin{array}{ll}
B_{1,1}(x-0)-B_{2,2}(x-0) & B_{1,2}(x-0)+B_{2,1}(x-0) \\
B_{1,2}(x-0)+B_{2,1}(x-0) & B_{2,2}(x-0)-B_{1,1}(x-0)
\end{array}\right), \text { etc. }
\end{aligned}
$$

The expansion (4.65) is uniform with respect to $\arg (z)$ for $|z| \rightarrow \infty$ in $C_{\varepsilon}$, and uniform in $x$ as long as $x$ varies in compact subsets of $\mathbb{R}$ intersected with the right and left Lebesgue set of $B^{(N-1)}$.

If one merely assumes Hypothesis 2.1 with $A=I_{2 m}, \alpha_{0}=\left(I_{m} 0\right)$, and $B \in$ $L_{\text {loc }}^{1}(\mathbb{R})^{2 m \times 2 m}$, then

$$
M\left(z, x, \alpha_{0}\right) \underset{\substack{|z| \rightarrow \infty \\ z \in C_{\varepsilon}}}{=}(i / 2) I_{2 m}+o(1)
$$


Again the asymptotic expansion (4.67) is uniform with respect to $\arg (z)$ for $|z| \rightarrow \infty$ in $C_{\varepsilon}$, and uniform in $x \in \mathbb{R}$ as long as $x$ varies in compact intervals.

The higher-order coefficients in (4.65) can be derived upon inserting (4.62) into (3.16a), taking (2.78) into account.

Theorems 4.7 and 4.8 (with $N \in \mathbb{N}$ ) are new even in the scalar case $m=1$ with respect to the regularity assumptions on $B$. For previous results in the case $m=1$ under stronger hypotheses on $B$, we refer to [33], [57], 661], [62], [98]. In particular, 57], 61], and 62] derived alternative high-energy expansions for the Weyl-Titchmarsh $m$-function in the case $m=1$.

Throughout this section we fixed $\alpha$ to be $\alpha_{0}=\left(I_{m} 0\right)$. The case of general $\alpha \in \mathbb{C}^{2 m \times m}$ satisfying (2.9) then follows from 2.61).

\section{A Local Uniqueness Result}

In this section we assume that $B$ is in the normal form given in Theorem 1.1 ,

$$
B(x)=\left(\begin{array}{cc}
B_{1,1}(x) & B_{1,2}(x) \\
B_{1,2}(x) & -B_{1,1}(x)
\end{array}\right),
$$

with $B_{1,1}$ and $B_{1,2}$ self-adjoint a.e. We prove fundamental new local uniqueness results for $B$ in terms of exponentially small differences of the Weyl-Titchmarsh matrices $M_{+}(z, x, \alpha)$ and $M(z, x, \alpha)$. These results, in turn, yield new global ramifications. We start with an auxiliary result concerning asymptotic expansions.

Lemma 5.1. Suppose $\alpha=\left(\begin{array}{ll}\alpha_{1} & \alpha_{2}\end{array}\right) \in \mathbb{C}^{m \times 2 m}$ satisfies (2.9), fix $x_{0}, y_{0} \in \mathbb{R}$ with $y_{0}>x_{0}$, and let $x \geq x_{0}$. Assume $A=I_{2 m}, B \in L^{1}\left(\left[x_{0}, \infty\right)\right)^{2 m \times 2 m}, \operatorname{supp}(B) \subseteq$ $\left[x_{0}, y_{0}\right]$, with $B$ in the normal form given in (5.1) a.e. on $\left(x_{0}, y_{0}\right)$. Then, the following asymptotic expansions hold for $\Theta\left(z, x, x_{0}, \alpha\right), \Phi\left(z, x, x_{0}, \alpha\right)$, and $U_{+}\left(z, x, x_{0}, \alpha\right)$ associated with (2.2a):

$$
\Theta\left(z, x, x_{0}, \alpha\right) \underset{\substack{|z| \rightarrow \infty \\
z \in \mathbb{C}_{+}}}{=} \frac{1}{2}\left(\begin{array}{c}
\alpha_{1}^{*}+i \alpha_{2}^{*} \\
-i\left(\alpha_{1}^{*}+i \alpha_{2}^{*}\right)
\end{array}\right) \exp \left(-i z\left(x-x_{0}\right)\right)(1+o(1)), \quad x>x_{0},
$$

$$
\Phi\left(z, x, x_{0}, \alpha\right) \underset{\substack{|z| \rightarrow \infty \\
z \in \mathbb{C}_{+}}}{=} \frac{i}{2}\left(\begin{array}{c}
-\alpha_{2}^{*}+i \alpha_{1}^{*} \\
-i\left(-\alpha_{2}^{*}+i \alpha_{1}^{*}\right)
\end{array}\right) \exp \left(-i z\left(x-x_{0}\right)\right)(1+o(1)), \quad x>x_{0},
$$

$$
U_{+}\left(z, x, x_{0}, \alpha\right) \underset{\substack{|z| \rightarrow \infty \\
z \in \mathbb{C}_{+}}}{=}\left(\begin{array}{c}
\alpha_{1}^{*}-i \alpha_{2}^{*} \\
i\left(\alpha_{1}^{*}-i \alpha_{2}^{*}\right)
\end{array}\right) \exp \left(i z\left(x-x_{0}\right)\right)(1+o(1)), \quad x \geq x_{0} .
$$

Next, we introduce the abbreviations

$$
C=-B_{1,2}-i B_{1,1}, \quad C^{*}=-B_{1,2}+i B_{1,1},
$$

and suppose in addition that

$$
\underset{y \in\left[x_{0}, y_{0}\right]}{\operatorname{ess} \sup }\left\|\int_{y}^{y_{0}} d x^{\prime} B\left(x^{\prime}\right) \exp \left(2 i z\left(x^{\prime}-y\right)\right)+\frac{1}{2 i z} B(y)\right\|_{\mathbb{C}^{2 m \times 2 m}} \underset{\substack{|z| \rightarrow \infty \\ z \in \rho_{+}}}{=} o\left(|z|^{-1}\right)
$$

along a ray $\rho_{+} \subset \mathbb{C}_{+}$, and that

$$
B_{1,1}^{2}, B_{1,2}^{2}, B_{1,1} B_{1,2}, B_{1,2} B_{1,1} \in L^{1}\left(\left[x_{0}, y_{0}\right]\right)^{m \times m} .
$$


Then,

$$
\begin{aligned}
& \Theta\left(z, x, x_{0}, \alpha\right) \underset{\substack{|z| \rightarrow \infty \\
z \in \rho_{+}}}{=}\left(\frac{1}{2}\left(\begin{array}{c}
\alpha_{1}^{*}+i \alpha_{2}^{*} \\
-i\left(\alpha_{1}^{*}+i \alpha_{2}^{*}\right)
\end{array}\right)-\frac{i}{4 z}\left(\begin{array}{c}
C\left(x_{0}\right)^{*}\left(\alpha_{1}^{*}-i \alpha_{2}^{*}\right) \\
-i C\left(x_{0}\right)^{*}\left(\alpha_{1}^{*}-i \alpha_{2}^{*}\right)
\end{array}\right)\right. \\
& -\frac{i}{4 z}\left(\begin{array}{c}
C(x)\left(\alpha_{1}^{*}+i \alpha_{2}^{*}\right) \\
i C(x)\left(\alpha_{1}^{*}+i \alpha_{2}^{*}\right)
\end{array}\right) \\
& \left.+\frac{i}{4 z} \int_{x_{0}}^{x} d x^{\prime}\left(\begin{array}{c}
C\left(x^{\prime}\right)^{*} C\left(x^{\prime}\right)\left(\alpha_{1}^{*}+i \alpha_{2}^{*}\right) \\
-i C\left(x^{\prime}\right)^{*} C\left(x^{\prime}\right)\left(\alpha_{1}^{*}+i \alpha_{2}^{*}\right)
\end{array}\right)\right) e^{-i z\left(x-x_{0}\right)}\left(1+o\left(|z|^{-1}\right)\right), \\
& \Phi>x_{0}, \\
& +\frac{1}{4 z}\left(\begin{array}{c}
C(x)\left(-\alpha_{2}^{*}+i \alpha_{1}^{*}\right) \\
i C(x)\left(-\alpha_{2}^{*}+i \alpha_{1}^{*}\right)
\end{array}\right) \\
& \left.-\frac{1}{4 z} \int_{x_{0}}^{x} d x^{\prime}\left(\begin{array}{c}
C\left(x^{\prime}\right)^{*} C\left(x^{\prime}\right)\left(-\alpha_{2}^{*}+i \alpha_{1}^{*}\right) \\
-i C\left(x^{\prime}\right)^{*} C\left(x^{\prime}\right)\left(-\alpha_{2}^{*}+i \alpha_{1}^{*}\right)
\end{array}\right)\right) e^{-i z\left(x-x_{0}\right)}\left(1+o\left(|z|^{-1}\right)\right), \\
& =\underset{z \in \rho_{+}}{=}\left(\frac{i}{2}\left(\begin{array}{c}
-\alpha_{2}^{*}+i \alpha_{1}^{*} \\
-i\left(-\alpha_{2}^{*}+i \alpha_{1}^{*}\right)
\end{array}\right)-\frac{1}{4 z}\left(\begin{array}{c}
C\left(x_{0}\right)^{*}\left(-\alpha_{2}^{*}-i \alpha_{1}^{*}\right) \\
-i C\left(x_{0}\right)^{*}\left(-\alpha_{2}^{*}-i \alpha_{1}^{*}\right)
\end{array}\right)\right. \\
& x>x_{0},
\end{aligned}
$$

whenever $x_{0}$ is a right Lebesgue point of $B$ and $x$ is a left Lebesgue point of $B$, and

$$
U_{+}\left(z, x, x_{0}, \alpha\right) \underset{\substack{|z| \rightarrow \infty \\
z \in \rho_{+}}}{=}\left(\left(\begin{array}{c}
\alpha_{1}^{*}-i \alpha_{2}^{*} \\
i\left(\alpha_{1}^{*}-i \alpha_{2}^{*}\right)
\end{array}\right)+\frac{i}{2 z}\left(\begin{array}{c}
\left(C(x)^{*}-C\left(x_{0}\right)^{*}\right)\left(\alpha_{1}^{*}-i \alpha_{2}^{*}\right) \\
-i\left(C(x)^{*}+C\left(x_{0}\right)^{*}\right)\left(\alpha_{1}^{*}-i \alpha_{2}^{*}\right)
\end{array}\right)\right.
$$

$$
\left.-\frac{i}{2 z} \int_{x_{0}}^{x} d x^{\prime}\left(\begin{array}{c}
C\left(x^{\prime}\right) C\left(x^{\prime}\right)^{*}\left(\alpha_{1}^{*}-i \alpha_{2}^{*}\right) \\
i C\left(x^{\prime}\right) C\left(x^{\prime}\right)^{*}\left(\alpha_{1}^{*}-i \alpha_{2}^{*}\right)
\end{array}\right)\right) e^{i z\left(x-x_{0}\right)}\left(1+o\left(|z|^{-1}\right)\right), \quad x \geq x_{0},
$$

whenever $x$ is a right Lebesgue point of $B$.

Proof. Since $x_{0}$ and $\alpha$ are fixed throughout this proof, we will temporarily suppress these variables whenever possible to simplify notation. Introducing

$$
\widehat{\Theta}(z, x)=2 \Theta(z, x) \exp \left(i z\left(x-x_{0}\right)\right),
$$

we can rewrite the Volterra integral equation for $\Theta$ (cf. (4.8)),

$$
\begin{aligned}
\Theta(z, x)= & \left(\begin{array}{l}
\alpha_{1}^{*} \cos \left(z\left(x-x_{0}\right)\right)+\alpha_{2}^{*} \sin \left(z\left(x-x_{0}\right)\right) \\
\alpha_{2}^{*} \cos \left(z\left(x-x_{0}\right)\right)-\alpha_{1}^{*} \sin \left(z\left(x-x_{0}\right)\right)
\end{array}\right) \\
& -\int_{x_{0}}^{x} d x^{\prime} K\left(z, x, x^{\prime}\right) J B\left(x^{\prime}\right) \Theta\left(z, x^{\prime}\right),
\end{aligned}
$$

in terms of that of $\widehat{\Theta}$ in the form

$$
\begin{aligned}
\widehat{\Theta}(z, x)= & \left(\begin{array}{c}
\alpha_{1}^{*}+i \alpha_{2}^{*} \\
-i\left(\alpha_{1}^{*}+i \alpha_{2}^{*}\right)
\end{array}\right)+\left(\begin{array}{c}
\alpha_{1}^{*}-i \alpha_{2}^{*} \\
i\left(\alpha_{1}^{*}-i \alpha_{2}^{*}\right)
\end{array}\right) \exp \left(2 i z\left(x-x_{0}\right)\right) \\
& -\frac{1}{2} \int_{x_{0}}^{x} d x^{\prime}\left(R\left(x^{\prime}\right) \exp \left(2 i z\left(x-x^{\prime}\right)\right)+S\left(x^{\prime}\right)\right) \widehat{\Theta}\left(z, x^{\prime}\right),
\end{aligned}
$$

where we abbreviated

$$
R=\left(\begin{array}{cc}
C & i C \\
i C & -C
\end{array}\right), \quad S=\left(\begin{array}{cc}
C^{*} & -i C^{*} \\
-i C^{*} & -C^{*}
\end{array}\right) .
$$


Using the elementary algebraic facts

$$
R\left(\begin{array}{c}
a \\
i a
\end{array}\right)=0, \quad R\left(\begin{array}{c}
b \\
-i b
\end{array}\right)=2\left(\begin{array}{c}
C b \\
i C b
\end{array}\right), \quad S\left(\begin{array}{c}
a \\
i a
\end{array}\right)=2\left(\begin{array}{c}
C^{*} a \\
-i C^{*} a
\end{array}\right), \quad S\left(\begin{array}{c}
b \\
-i b
\end{array}\right)=0
$$

for any $a, b \in \mathbb{C}^{m \times m}$, iterating (5.13) yields

$$
\begin{gathered}
\widehat{\Theta}(z, x)=\left(\begin{array}{c}
\alpha_{1}^{*}+i \alpha_{2}^{*} \\
-i\left(\alpha_{1}^{*}+i \alpha_{2}^{*}\right)
\end{array}\right)+\left(\begin{array}{c}
\alpha_{1}^{*}-i \alpha_{2}^{*} \\
i\left(\alpha_{1}^{*}+i \alpha_{2}^{*}\right)
\end{array}\right) e^{2 i z\left(x-x_{0}\right)} \\
+\sum_{m=1}^{\infty}(-2)^{-m} \int_{x_{0}}^{x} d \xi_{1}\left(R\left(\xi_{1}\right) e^{2 i z\left(x-\xi_{1}\right)}+S\left(\xi_{1}\right)\right) \\
\times \int_{x_{0}}^{\xi_{1}} d \xi_{2}\left(R\left(\xi_{2}\right) e^{2 i z\left(\xi_{1}-\xi_{2}\right)}+S\left(\xi_{2}\right)\right) \\
\quad \ldots \int_{x_{0}}^{\xi_{m-2}} d \xi_{m-1}\left(R\left(\xi_{m-1}\right) e^{2 i z\left(\xi_{m-2}-\xi_{m-1}\right)}+S\left(\xi_{m-1}\right)\right) \\
\times \int_{x_{0}}^{\xi_{m-1}} d \xi_{m}\left(R\left(\xi_{m}\right)\left(\begin{array}{c}
\alpha_{1}^{*}-i \alpha_{2}^{*} \\
-i\left(\alpha_{1}^{*}-i \alpha_{2}^{*}\right)
\end{array}\right) e^{2 i z\left(\xi_{m-1}-\xi_{m}\right)}\right. \\
\left.+S\left(\xi_{m}\right)\left(\begin{array}{c}
\alpha_{1}^{*}+i \alpha_{2}^{*} \\
i\left(\alpha_{1}^{*}+i \alpha_{2}^{*}\right)
\end{array}\right) e^{2 i z\left(\xi_{m}-x_{0}\right)}\right) .
\end{gathered}
$$

Applying the Riemann-Lebesgue lemma to (5.16) then proves (5.2), assuming $B \in$ $L^{1}\left(\left[x_{0}, \infty\right)\right)^{2 m \times 2 m}$ only. Assuming also (5.6) and (5.7) one can compute the next term in the asymptotic expansion (5.2), and then obtain (5.8) using (5.16) and the finite-interval variant of (4.1), whenever $x_{0}$ is a right Lebesgue point of $B$ and $x$ is a left Lebesgue point of $B$.

Exactly the same arguments apply to $\Phi$. Introducing

$$
\widehat{\Phi}(z, x)=2 \Phi(z, x) \exp \left(i z\left(x-x_{0}\right)\right)
$$

one can rewrite the Volterra integral equation for $\Phi$,

$$
\begin{aligned}
\Phi(z, x)= & \left(\begin{array}{c}
-\alpha_{2}^{*} \cos \left(z\left(x-x_{0}\right)\right)+\alpha_{1}^{*} \sin \left(i z\left(x-x_{0}\right)\right) \\
\alpha_{1}^{*} \cos \left(i z\left(x-x_{0}\right)\right)+\alpha_{2}^{*} \sin \left(z\left(x-x_{0}\right)\right)
\end{array}\right) \\
& -\int_{x_{0}}^{x} d x^{\prime} K\left(z, x, x^{\prime}\right) J B\left(x^{\prime}\right) \Phi_{j}\left(z, x^{\prime}\right),
\end{aligned}
$$

in terms of that of $\widehat{\Phi}$ in the form

$$
\begin{aligned}
\widehat{\Phi}(z, x)= & i\left(\begin{array}{c}
-\alpha_{2}^{*}+i \alpha_{1}^{*} \\
-i\left(-\alpha_{2}^{*}+i \alpha_{1}^{*}\right)
\end{array}\right)-i\left(\begin{array}{c}
-\alpha_{2}^{*}-i \alpha_{1}^{*} \\
i\left(-\alpha_{2}^{*}-i \alpha_{1}^{*}\right)
\end{array}\right) \exp \left(2 i z\left(x-x_{0}\right)\right) \\
& -\frac{1}{2} \int_{x_{0}}^{x}\left(R\left(x^{\prime}\right) \exp \left(2 i z\left(x-x^{\prime}\right)\right)+S\left(x^{\prime}\right)\right) \widehat{\Phi}\left(z, x^{\prime}\right) .
\end{aligned}
$$


Iterating (5.19) and taking into account (5.15) yields

$$
\begin{gathered}
\widehat{\Phi}(z, x)=i\left(\begin{array}{c}
-\alpha_{2}^{*}+i \alpha_{1}^{*} \\
-i\left(-\alpha_{2}^{*}+i \alpha_{1}^{*}\right)
\end{array}\right)-i\left(\begin{array}{c}
-\alpha_{2}^{*}-i \alpha_{1}^{*} \\
i\left(-\alpha_{2}^{*}-i \alpha_{1}^{*}\right)
\end{array}\right) e^{2 i z\left(x-x_{0}\right)} \\
+\sum_{m=1}^{\infty}(-2)^{-m} \int_{x_{0}}^{x} d \xi_{1}\left(R\left(\xi_{1}\right) e^{2 i z\left(x-\xi_{1}\right)}+S\left(\xi_{1}\right)\right) \\
\times \int_{x_{0}}^{\xi_{1}} d \xi_{2}\left(R\left(\xi_{2}\right) e^{2 i z\left(\xi_{1}-\xi_{2}\right)}+S\left(\xi_{2}\right)\right) \\
\ldots \int_{x_{0}}^{\xi_{m-2}} d \xi_{m-1}\left(R\left(\xi_{m-1}\right) e^{2 i z\left(\xi_{m-2}-\xi_{m-1}\right)}+S\left(\xi_{m-1}\right)\right) \\
\times \int_{x_{0}}^{\xi_{m-1}} d \xi_{m}\left(i R\left(\xi_{m}\right)\left(\begin{array}{c}
-\alpha_{2}^{*}+i \alpha_{1}^{*} \\
-i\left(-\alpha_{2}^{*}+i \alpha_{1}^{*}\right)
\end{array}\right) e^{2 i z\left(\xi_{m-1}-\xi_{m}\right)}\right. \\
\left.-i S\left(\xi_{m}\right)\left(\begin{array}{c}
-\alpha_{2}^{*}-i \alpha_{1}^{*} \\
i\left(-\alpha_{2}^{*}-i \alpha_{1}^{*}\right)
\end{array}\right) e^{2 i z\left(\xi_{m}-x_{0}\right)}\right) .
\end{gathered}
$$

Applying the Riemann-Lebesgue lemma to (5.20), one proves (5.3), assuming $B \in$ $L^{1}\left(\left[x_{0}, \infty\right)\right)^{2 m \times 2 m}$ only. Assuming also (5.6) and (5.7), one can compute the next term in the asymptotic expansion (5.3) and then obtain (5.9) using (5.20) and the finite-interval variant of (4.1), whenever $x_{0}$ is a right Lebesgue point of $B$ and $x$ is a left Lebesgue point of $B$.

Finally, we turn to $U_{+}(z, x)$. Introducing

$$
\widetilde{V}_{+}(z, x)=\widetilde{U}_{+}(z, x) \exp \left(-i z\left(x-x_{0}\right)\right)
$$

we can rewrite the Volterra integral equation for $\widetilde{U}_{+}$,

$$
\widetilde{U}_{+}(z, x)=\left(\begin{array}{c}
\alpha_{1}^{*}-i \alpha_{2}^{*} \\
i\left(\alpha_{1}^{*}-i \alpha_{2}^{*}\right)
\end{array}\right) \exp \left(i z\left(x-x_{0}\right)\right)+\int_{x}^{\infty} d x^{\prime} K\left(z, x, x^{\prime}\right) J B\left(x^{\prime}\right) \widetilde{U}_{+}\left(z, x^{\prime}\right),
$$

in terms of that of $\widetilde{V}_{+}$in the form

$$
\widetilde{V}_{+}(z, x)=\left(\begin{array}{c}
\alpha_{1}^{*}-i \alpha_{2}^{*} \\
i\left(\alpha_{1}^{*}-i \alpha_{2}^{*}\right)
\end{array}\right)+\frac{1}{2} \int_{x}^{y_{0}} d x^{\prime}\left(R\left(x^{\prime}\right)+S\left(x^{\prime}\right) \exp \left(2 i z\left(x^{\prime}-x\right)\right)\right) \widetilde{V}_{+}\left(z, x^{\prime}\right) .
$$


Iterating (5.23) and taking into account (5.15) yields

$$
\begin{aligned}
\widetilde{V}_{+}(z, x)= & \left(\begin{array}{c}
\alpha_{1}^{*}-i \alpha_{2}^{*} \\
i\left(\alpha_{1}^{*}-i \alpha_{2}^{*}\right)
\end{array}\right)+\sum_{k=1}^{\infty} 2^{-2 k} \int_{x}^{y_{0}} d \xi_{1} R\left(\xi_{1}\right) \int_{\xi_{1}}^{y_{0}} d \xi_{2} S\left(\xi_{2}\right) e^{2 i z\left(\xi_{2}-\xi_{1}\right)} \\
& \times \int_{\xi_{2}}^{y_{0}} d \xi_{3} R\left(\xi_{3}\right) \cdots \int_{\xi_{2 k-2}}^{y_{0}} d \xi_{2 k-1} R\left(\xi_{2 k-1}\right) \\
& \times \int_{\xi_{2 k-1}}^{y_{0}} d \xi_{2 k} S\left(\xi_{m}\right)\left(\begin{array}{c}
\alpha_{1}^{*}-i \alpha_{2}^{*} \\
i\left(\alpha_{1}^{*}-i \alpha_{2}^{*}\right)
\end{array}\right) e^{i z\left(\xi_{2 k}-\xi_{2 k-1}\right)} \\
& +\sum_{\ell=0}^{\infty} 2^{-2 \ell+1} \int_{x}^{y_{0}} d \xi_{1} S\left(\xi_{1}\right) e^{2 i z\left(\xi_{1}-x\right)} \int_{\xi_{1}}^{y_{0}} d \xi_{2} R\left(\xi_{2}\right) \\
& \times \int_{\xi_{2}}^{y_{0}} d \xi_{3} S\left(\xi_{3}\right) e^{2 i z\left(\xi_{3}-\xi_{2}\right) \ldots \int_{\xi_{2 \ell-1}}^{y_{0}} d \xi_{2 \ell} R\left(\xi_{2 \ell}\right)} \\
& \times \int_{\xi_{2 \ell}}^{y_{0}} d \xi_{2 \ell+1} S\left(\xi_{2 \ell+1}\right)\left(\begin{array}{c}
\alpha_{1}^{*}-i \alpha_{2}^{*} \\
i\left(\alpha_{1}^{*}-i \alpha_{2}^{*}\right)
\end{array}\right) e^{i z\left(\xi_{2 \ell+1}-\xi_{2 \ell}\right)} .
\end{aligned}
$$

Next, we take into account the different normalizations of $U_{+}$and $\widetilde{U}_{+}$. Using $U_{+}\left(z, x_{0}\right)=\left[I_{m} M_{+}\left(z, x_{0}\right)^{t}\right]^{t}(\mathrm{cf}$. (2.70) $)$ and $\Psi\left(z, x_{0}, x_{0}, \alpha_{0}\right)=I_{2 m}$, one readily verifies the relationship

$$
u_{+, 1}(z, x)=\widetilde{u}_{+, 1}(z, x) \widetilde{u}_{+, 1}\left(z, x_{0}\right)^{-1}, \quad u_{+, 2}(z, x)=\widetilde{u}_{+, 2}(z, x) \widetilde{u}_{+, 1}\left(z, x_{0}\right)^{-1} .
$$

Thus, applying the Riemann-Lebesgue lemma to (5.24) then proves (5.4) (in agreement with (4.26) $)$, assuming $B \in L^{1}\left(\left[x_{0}, \infty\right)\right)^{2 m \times 2 m}$ only. Assuming also (5.6) and (5.7), one can compute the next term in the asymptotic expansion (5.4), and then obtain (5.10) using (5.24) and (4.1), whenever $x$ is a right Lebesgue point of $B$.

In the special case $m=1$ (and for $\alpha=\left(\begin{array}{ll}1 & 0\end{array}\right)$ ), the expansion (5.10) was stated in 55.

Next, we note an elementary result concerning the boundary data independence of exponentially close Weyl-Titchmarsh matrices.

Lemma 5.2. Fix $x_{0} \in \mathbb{R}$ and suppose $A_{j}=I_{2 m}, B_{j}=B_{j}^{*} \in L^{1}\left(\left[x_{0}, x_{0}+R\right]\right)^{2 m \times 2 m}$ for all $R>0$. Denote by $M_{+, j}(z, x, \alpha), x \geq x_{0}$, the unique Weyl-Titchmarsh matrices corresponding to the half-line Dirac-type operators $D_{+, j}(\alpha), j=1,2$, in (2.89). Fix an $\hat{\alpha} \in \mathbb{C}^{m \times 2 m}$ satisfying (2.9), and assume that for all $\varepsilon>0$

$$
\left\|M_{+, 1}\left(z, x_{0}, \hat{\alpha}\right)-M_{+, 2}\left(z, x_{0}, \hat{\alpha}\right)\right\|_{\mathbb{C}^{m \times m}} \underset{\substack{|z| \rightarrow \infty \\ z \in \rho_{+}}}{=} O\left(e^{-2 \operatorname{Im}(z)(a-\varepsilon)}\right)
$$

along some ray $\rho_{+} \subset \mathbb{C}_{+}$. Then, for all $\alpha \in \mathbb{C}^{m \times 2 m}$ satisfying (2.9) and for all $\varepsilon>0$,

$$
\left\|M_{+, 1}\left(z, x_{0}, \alpha\right)-M_{+, 2}\left(z, x_{0}, \alpha\right)\right\|_{\mathbb{C}^{m \times m}} \underset{\substack{|z| \rightarrow \infty \\ z \in \rho_{+}}}{=} O\left(e^{-2 \operatorname{Im}(z)(a-\varepsilon)}\right)
$$

along the ray $\rho_{+}$. 
Proof. Using (2.58) and (2.61), one estimates

$$
\begin{aligned}
& \left\|M_{+, 1}\left(z, x_{0}, \alpha\right)-M_{+, 2}\left(z, x_{0}, \alpha\right)\right\|_{\mathbb{C}^{m \times m}} \\
& =\left\|M_{+, 1}\left(\bar{z}, x_{0}, \alpha\right)^{*}-M_{+, 2}\left(z, x_{0}, \alpha\right)\right\|_{\mathbb{C}^{m \times m}} \\
& \leq\left\|\left[\hat{\alpha} \alpha^{*}-M_{+, 1}\left(\bar{z}, x_{0}, \hat{\alpha}\right)^{*} \hat{\alpha} J \alpha^{*}\right]^{-1}\right\|_{\mathbb{C}^{m \times m}} \\
& \quad \times\left\|M_{+, 1}\left(z, x_{0}, \hat{\alpha}\right)-M_{+, 2}\left(z, x_{0}, \hat{\alpha}\right)\right\|_{\mathbb{C}^{m \times m}} \\
& \quad \times\left\|\left[\alpha \hat{\alpha}^{*}+\alpha J \hat{\alpha}^{*} M_{+, 2}\left(z, x_{0}, \hat{\alpha}\right)\right]^{-1}\right\|_{\mathbb{C}^{m \times m}},
\end{aligned}
$$

since by (2.13)

$$
\hat{\alpha} J \alpha^{*} \alpha \hat{\alpha}^{*}+\hat{\alpha} \alpha^{*} \alpha J \hat{\alpha}^{*}=0, \quad \hat{\alpha} \alpha^{*} \alpha \hat{\alpha}^{*}-\hat{\alpha} J \alpha^{*} \alpha J \hat{\alpha}^{*}=I_{m} .
$$

Moreover, since

$$
\left[\hat{\alpha} \alpha^{*}-i \hat{\alpha} J \alpha^{*}\right]\left[\hat{\alpha} \alpha^{*}-i \hat{\alpha} J \alpha^{*}\right]^{*}=I_{m},
$$

by (5.29), one infers (5.27) from (5.28) and $M_{+, j}\left(z, x_{0}, \alpha\right)=i I_{m}+o(1)$ as $|z| \rightarrow \infty$, $z \in \mathbb{C}_{+}, j=1,2$ (cf. (3.1)).

Our principal new local uniqueness result for Dirac-type operators in terms of Weyl-Titchmarsh matrices then reads as follows.

Theorem 5.3. Fix $x_{0} \in \mathbb{R}$ and suppose $A_{j}=I_{2 m}, B_{j} \in L^{1}\left(\left[x_{0}, x_{0}+R\right]\right)^{2 m \times 2 m}$ for all $R>0$. Suppose also that $B_{j}$ is in the normal form given in (5.1) a.e. on $\left(x_{0}, \infty\right), j=1,2$. Denote by $M_{j,+}(z, x, \alpha), x \geq x_{0}$, the unique Weyl-Titchmarsh matrices corresponding to the half-line Dirac-type operators $D_{+, j}(\alpha), j=1,2$, in (2.89). Then,

$$
\text { if for some } a>0, B_{1}(x)=B_{2}(x) \text { for a.e. } x \in\left(x_{0}, x_{0}+a\right),
$$

one obtains

$$
\left\|M_{1,+}\left(z, x_{0}, \alpha\right)-M_{2,+}\left(z, x_{0}, \alpha\right)\right\|_{\mathbb{C}^{m \times m}} \underset{\substack{|z| \rightarrow \infty \\ z \in \rho_{+}}}{=} O\left(e^{-2 \operatorname{Im}(z) a}\right)
$$

along any ray $\rho_{+} \subset \mathbb{C}_{+}$with $0<\arg (z)<\pi$ and for all $\alpha \in \mathbb{C}^{m \times 2 m}$ satisfying (2.9). Conversely, fix an $\hat{\alpha} \in \mathbb{C}^{m \times 2 m}$ satisfying (2.9), and if $m>1$, assume in addition that $B_{j} \in L^{\infty}\left(\left[x_{0}, x_{0}+a\right]\right)^{2 m \times 2 m}, j=1,2$. Moreover, suppose that for all $\varepsilon>0$,

$$
\left\|M_{1,+}\left(z, x_{0}, \hat{\alpha}_{1}\right)-M_{2,+}\left(z, x_{0}, \hat{\alpha}_{1}\right)\right\|_{\mathbb{C}^{m \times m}} \underset{\substack{|z| \rightarrow \infty \\ z \in \rho_{+}, \ell}}{=} O\left(e^{-2 \operatorname{Im}(z)(a-\varepsilon)}\right), \quad \ell=1,2,
$$

along a ray $\rho_{+, 1} \subset \mathbb{C}_{+}$with $0<\arg (z)<\pi / 2$ and along a ray $\rho_{+, 2} \subset \mathbb{C}_{+}$with $\pi / 2<\arg (z)<\pi$. Then

$$
B_{1}(x)=B_{2}(x) \text { for a.e. } x \in\left[x_{0}, x_{0}+a\right] .
$$

Proof. Since (5.32) follows from Theorem 5.6 and Lemma 5.2 it suffices to focus on the proof of (5.34). Moreover, applying Theorem 4.6, we may without loss of generality assume for the rest of the proof that

$$
\operatorname{supp}\left(B_{j}\right) \subseteq\left[x_{0}, x_{0}+a\right], \quad j=1,2 .
$$

In the following, we will adapt the principal ingredients of a recent proof of the local Borg-Marchenko uniqueness theorem for scalar Schrödinger operators (i.e., for $m=$ 1), by Bennewitz [13], to the current Dirac-type situation. First we recall that by 
Lemma 5.2] (5.33) holds along the rays $\rho_{+, j}, j=1,2$, for all $\alpha=\left(\alpha_{1} \alpha_{2}\right) \in \mathbb{C}^{m \times 2 m}$ satisfying (2.9). To simplify notation in the following, we will again suppress $x_{0}$ and $\alpha$ whenever possible, and hence abbreviate $\Theta\left(z, x, x_{0}, \alpha\right), \Phi\left(z, x, x_{0}, \alpha\right)$, and $U_{j,+}\left(z, x, x_{0}, \alpha\right)$ by $\Theta(z, x), \Phi(z, x)$, and $U_{j,+}(z, x)$, respectively. Next, denoting in obvious notation by

$\Theta_{j}(z, x)=\left(\begin{array}{c}\theta_{j, 1}(z, x) \\ \theta_{j, 2}(z, x)\end{array}\right), \quad \Phi_{j}(z, x)=\left(\begin{array}{c}\phi_{j, 1}(z, x) \\ \phi_{j, 2}(z, x)\end{array}\right), \quad U_{j,+}(z, x)=\left(\begin{array}{l}u_{j,+, 1}(z, x) \\ u_{j,+, 2}(z, x)\end{array}\right)$,

$$
j=1,2, x \geq x_{0}
$$

the solutions associated with $B_{j}, j=1,2$, which are defined in (2.14b) and (2.17), we introduce

$$
g_{j, k}(z, x)=\phi_{j, k}(z, x) u_{j,+, k}(\bar{z}, x)^{*}, \quad j, k \in\{1,2\}, x \geq x_{0} .
$$

Using the asymptotic expansions (5.2)-(5.4) for $\Theta_{j}(z, x), \Phi_{j}(z, x)$, and $U_{j,+}(z, x)$, and the analogous ones for $\Theta_{j}(\bar{z}, x)^{*}, \Phi_{j}(\bar{z}, x)^{*}$, and $U_{j,+}(\bar{z}, x)^{*}$, one verifies for each fixed $x>x_{0}$,

$$
g_{j, k}(z, x) \underset{\substack{|z| \rightarrow \infty \\ z \in \mathbb{C}_{+}}}{=}(i / 2) I_{m}+o(1), \quad j, k \in\{1,2\}
$$

assuming $B_{j} \in L^{1}\left(\left[x_{0}, x_{0}+R\right]\right)^{2 m \times 2 m}$ for all $R>0, j=1,2$, only. Next, using the fact that for each fixed $x>x_{0}$,

$$
\begin{aligned}
& \phi_{1, k}(z, x)^{-1} \phi_{2, k}(z, x)=I_{\substack{|z| \rightarrow \infty \\
z \in \mathbb{C}_{+}}}=I_{m}+o(1), \quad k=1,2, \\
&\left(u_{1,+, k}(\bar{z}, x)^{*}\right)^{-1} u_{2,+, k}(\bar{z}, x)^{*} \underset{\substack{|z| \rightarrow \infty \\
z \in \mathbb{C}_{+}}}{=} I_{m}+o(1), \quad k=1,2,
\end{aligned}
$$

by (5.3) and (5.4) one concludes that

$$
\begin{aligned}
& \phi_{1, k}(z, x) u_{2,+, j}(\bar{z}, x)^{*}-u_{1,+, k}(z, x) \phi_{2, k}(\bar{z}, x)^{*} \\
& =\phi_{1, k}(z, x) \theta_{2, k}(\bar{z}, x)^{*}-\theta_{1, k}(z, x) \phi_{2, k}(\bar{z}, x)^{*} \\
& \quad+\phi_{1, k}(z, x)\left(M_{2,+}(z)-M_{1,+}(z)\right) \phi_{2, k}(\bar{z}, x)^{*} \underset{\substack{\mid z) \rightarrow \infty \\
z \in \mathbb{C}_{+}}}{=} o(1),
\end{aligned}
$$

using (5.38), (5.40), and $M_{2,+}(\bar{z})^{*}=M_{2,+}(z)$. Combining hypothesis (5.33) and (5.3), one infers that

$$
\left\|\phi_{1, k}(z, x)\left(M_{2,+}(z)-M_{1,+}(z)\right) \phi_{2, k}(\bar{z}, x)^{*}\right\| \underset{\substack{|z| \rightarrow \rightarrow \infty \\ z \in \rho_{+, \ell}}}{=} o(1), \quad x \in\left(x_{0}, x_{0}+a\right)
$$

along the rays $\rho_{+, \ell}, \ell=1,2$. Thus, (5.41) implies that

$$
\left\|\phi_{1, k}(z, x) \theta_{2, k}(\bar{z}, x)^{*}-\theta_{1, k}(z, x) \phi_{2, k}(\bar{z}, x)^{*}\right\| \underset{\substack{|z| \rightarrow \infty \\ z \in \rho_{+, \ell}}}{=} o(1), \quad x \in\left(x_{0}, x_{0}+a\right),
$$

along the rays $\rho_{+, \ell}, \ell=1,2$. The analogous estimate (5.43) holds along the complex conjugate rays $\bar{\rho}_{+, \ell}, \ell=1,2$, in the lower complex half-plane $\mathbb{C}_{-}$. To simplify notation, we denote the open sector generated by $\rho_{+, 1}$ and its complex conjugate $\bar{\rho}_{+, 1}$ by $\mathcal{S}_{1}$, the open sector generated by the $\rho_{+, 2}$ and its complex conjugate $\bar{\rho}_{+, 2}$ 
by $\mathcal{S}_{2}$, the remaining sector in $\mathbb{C}_{+}$by $\mathcal{S}_{3}$, and its complex conjugate sector in $\mathbb{C}_{-}$ by $\mathcal{S}_{4}$. Thus, one obtains a partition of $\mathbb{C}$ into

$$
\mathbb{C}=\bigcup_{\ell=1}^{4} \overline{\mathcal{S}_{\ell}},
$$

where each sector $\mathcal{S}_{\ell}, 1 \leq \ell \leq 4$, has opening angle strictly less than $\pi$. Since (each matrix element of) the expression under the norm in (5.43) is entire and of order less than or equal to one, one can apply the Phragmén-Lindelöf principle (cf., e.g., 101, No. 322, pp. 166-167, 379]) to each sector $\mathcal{S}_{\ell}, 1 \leq \ell \leq 4$, and obtain that each matrix element under the norm in (5.43) is uniformly bounded in each sector and hence on all of $\mathbb{C}$. By Liouville's theorem, these matrix elements are all equal to certain constants. By the right-hand side of (5.43), these constants all vanish. Thus, we have proved that

$$
\phi_{1, k}(z, x) \theta_{2, k}(\bar{z}, x)^{*}=\theta_{1, k}(z, x) \phi_{2, k}(\bar{z}, x)^{*} \text { for all } x \in\left(x_{0}, x_{0}+a\right),
$$

and hence

$$
\phi_{1, k}(z, x)^{-1} \theta_{1, k}(z, x)=\theta_{2, k}(\bar{z}, x)^{*}\left(\phi_{2, k}(\bar{z}, x)^{*}\right)^{-1} \text { for all } x \in\left(x_{0}, x_{0}+a\right) .
$$

Differentiating $\phi_{j, k}(z, x)^{-1} \theta_{j, k}(z, x), j, k=1,2$, with respect to $x$ yields

$$
\begin{aligned}
& \left(\phi_{j, 1}(z, x)^{-1} \theta_{j, 1}(z, x)\right)^{\prime} \\
& =\phi_{j, 1}(z, x)^{-1}\left(\left(B_{j}\right)_{1,1}(x)-z\right)\left(\phi_{j, 2}(z, x) \phi_{j, 1}(z, x)^{-1} \theta_{j, 1}(z, x)-\theta_{j, 2}(z, x)\right) \\
& \left(\phi_{j, 2}(z, x)^{-1} \theta_{j, 2}(z, x)\right)^{\prime} \\
& =\phi_{j, 2}(z, x)^{-1}\left(\left(B_{j}\right)_{1,1}(x)+z\right)\left(\phi_{j, 1}(z, x) \phi_{j, 2}(z, x)^{-1} \theta_{j, 2}(z, x)-\theta_{j, 1}(z, x)\right)
\end{aligned}
$$

Multiplying (5.47) by $\phi_{j, 1}(\bar{z}, x)^{*}\left(\phi_{j, 1}(\bar{z}, x)^{*}\right)^{-1}$ and using (2.94) and (2.96), and similarly, multiplying (5.48) by $\phi_{j, 2}(\bar{z}, x)^{*}\left(\phi_{j, 2}(\bar{z}, x)^{*}\right)^{-1}$ and using (2.95) and (2.97) then yields

$$
\begin{aligned}
& \left(\phi_{j, 1}(z, x)^{-1} \theta_{j, 1}(z, x)\right)^{\prime}=\phi_{j, 1}(z, x)^{-1}\left(\left(B_{j}\right)_{1,1}(x)-z\right)\left(\phi_{j, 1}(\bar{z}, x)^{*}\right)^{-1} \\
& \left(\phi_{j, 2}(z, x)^{-1} \theta_{j, 2}(z, x)\right)^{\prime}=\phi_{j, 2}(z, x)^{-1}\left(\left(B_{j}\right)_{1,1}(x)+z\right)\left(\phi_{j, 2}(\bar{z}, x)^{*}\right)^{-1} .
\end{aligned}
$$

In exactly the same way one derives

$$
\begin{aligned}
& \left(\theta_{j, 1}(\bar{z}, x)^{*}\left(\phi_{j, 1}(\bar{z}, x)^{*}\right)^{-1}\right)^{\prime} \\
& =\left(\theta_{j, 1}(\bar{z}, x)^{*}\left(\phi_{j, 1}(\bar{z}, x)^{*}\right)^{-1} \phi_{j, 2}(\bar{z}, x)^{*}-\theta_{2}(\bar{z}, x)^{*}\right)\left(\left(B_{j}\right)_{1,1}(x)-z\right)\left(\phi_{j, 1}(\bar{z}, x)^{*}\right)^{-1} \\
& =\phi_{j, 1}(z, x)^{-1}\left(\left(B_{j}\right)_{1,1}(x)-z\right)\left(\phi_{j, 1}(\bar{z}, x)^{*}\right)^{-1}
\end{aligned}
$$

$$
\begin{aligned}
& \left(\theta_{j, 2}(\bar{z}, x)^{*}\left(\phi_{j, 2}(\bar{z}, x)^{*}\right)^{-1}\right)^{\prime} \\
& =\left(\theta_{j, 2}(\bar{z}, x)^{*}\left(\phi_{j, 2}(\bar{z}, x)^{*}\right)^{-1} \phi_{j, 1}(\bar{z}, x)^{*}-\theta_{j, 1}(\bar{z}, x)^{*}\right)\left(\left(B_{j}\right)_{1,1}(x)+z\right)\left(\phi_{j, 2}(\bar{z}, x)^{*}\right)^{-1} \\
& =\phi_{j, 2}(z, x)^{-1}\left(\left(B_{j}\right)_{1,1}(x)+z\right)\left(\phi_{j, 2}(\bar{z}, x)^{*}\right)^{-1}
\end{aligned}
$$


using (2.90)-(2.93). Thus, (5.46) implies

$$
\begin{aligned}
& \phi_{1,1}(\bar{z}, x)^{*}\left(\left(B_{1}\right)_{1,1}(x)-z\right)^{-1} \phi_{1,1}(z, x)=\phi_{2,1}(\bar{z}, x)^{*}\left(\left(B_{2}\right)_{1,1}(x)-z\right)^{-1} \phi_{2,1}(z, x), \\
& (5.54) \\
& \phi_{1,2}(\bar{z}, x)^{*}\left(\left(B_{1}\right)_{1,1}(x)+z\right)^{-1} \phi_{1,2}(z, x)=\phi_{2,2}(\bar{z}, x)^{*}\left(\left(B_{2}\right)_{1,1}(x)+z\right)^{-1} \phi_{2,2}(z, x),
\end{aligned}
$$

$$
\theta_{1,1}(\bar{z}, x)^{*}\left(\left(B_{1}\right)_{1,1}(x)-z\right)^{-1} \theta_{1,1}(z, x)=\theta_{2,1}(\bar{z}, x)^{*}\left(\left(B_{2}\right)_{1,1}(x)-z\right)^{-1} \theta_{2,1}(z, x),
$$

$$
\theta_{1,2}(\bar{z}, x)^{*}\left(\left(B_{1}\right)_{1,1}(x)+z\right)^{-1} \theta_{1,2}(z, x)=\theta_{2,2}(\bar{z}, x)^{*}\left(\left(B_{2}\right)_{1,1}(x)+z\right)^{-1} \theta_{2,2}(z, x)
$$

for a.e. $x \in\left(x_{0}, x_{0}+a\right)$. Thus far we have only used $B_{j} \in L^{1}\left(\left[x_{0}, x_{0}+R\right]\right)^{2 m \times 2 m}$ for all $R>0, j=1,2$, and (5.35).

In the special case $m=1$, each of the equations (5.53)-(5.56) allows for the completion of the proof of (5.34). Indeed, using the fact that

$$
\overline{\phi_{j, k}(\bar{z}, x)}=\phi_{j, k}(z, x), \quad \overline{\theta_{j, k}(\bar{z}, x)}=\theta_{j, k}(z, x), \quad j, k \in\{1,2\},
$$

and taking for instance (5.53), one infers, for a.e. $x \in\left(x_{0}, x_{0}+a\right)$, that

$$
\frac{\phi_{1,1}(z, x)^{2}}{\phi_{2,1}(z, x)^{2}}=\frac{\left(B_{1}\right)_{1,1}(x)-z}{\left(B_{2}\right)_{1,1}(x)-z} .
$$

Since all zeros (and poles) of the left-hand side of (5.58) have even multiplicity, while all zeros (and poles) of the right-hand side of (5.57) are simple, one concludes, assuming only that $B_{j} \in L^{1}\left(\left[x_{0}, x_{0}+R\right]\right)^{2 \times 2}$ for all $R>0, j=1,2$, that

$$
\left(B_{1}\right)_{1,1}(x)=\left(B_{2}\right)_{1,1}(x) \text { for a.e. } x \in\left(x_{0}, x_{0}+a\right) .
$$

Thus for the case $m=1$, we see by (5.53), (5.54), (5.57), and (5.59), for a.e. $x \in$ $\left(x_{0}, x_{0}+a\right)$, that

$$
\phi_{1, k}^{2}(z, x)=\phi_{2, k}^{2}(z, x), \quad k=1,2 .
$$

Now, (2.93), (5.46), and (5.57) show, for a.e. $x \in\left(x_{0}, x_{0}+a\right)$, that

$$
\begin{aligned}
\phi_{1,1}(z, x) \phi_{1,2}(z, x) & =\frac{\phi_{1,1}(z, x)}{\theta_{1,1}(z, x)}-\frac{\phi_{1,2}(z, x)}{\theta_{1,2}(z, x)} \\
& =\frac{\phi_{2,1}(z, x)}{\theta_{2,1}(z, x)}-\frac{\phi_{2,2}(z, x)}{\theta_{2,2}(z, x)}=\phi_{2,1}(z, x) \phi_{2,2}(z, x) .
\end{aligned}
$$

By (2.2a) we see that

$\left(\phi_{j, 1}^{2}(z, x)\right)^{\prime}=2\left(z-\left(B_{j}(x)\right)_{1,1}\right) \phi_{j, 1}(z, x) \phi_{j, 2}(z, x)+\left(B_{j}(x)\right)_{1,2} \phi_{j, 1}^{2}(z, x), \quad j=1,2$.

Thus, by (5.59), (5.60), and (5.61),

$$
\left(B_{1}(x)\right)_{1,2}=\left(B_{2}(x)\right)_{1,2} \text { for a.e. } x \in\left(x_{0}, x_{0}+a\right) .
$$

Together, (5.59) and (5.63) imply (5.34) in the special case $m=1$.

Unfortunately, the case $m>1$ appears to be quite a bit more involved. To deal with this case we first note that taking determinants in (5.53) yields

$$
\frac{\operatorname{det}\left(\phi_{1,1}\left(\bar{z}, x, x_{0}, \alpha\right)^{*}\right) \operatorname{det}\left(\phi_{1,1}\left(z, x, x_{0}, \alpha\right)\right)}{\operatorname{det}\left(\phi_{2,1}\left(\bar{z}, x, x_{0}, \alpha\right)^{*}\right) \operatorname{det}\left(\phi_{2,1}\left(z, x, x_{0}, \alpha\right)\right.}=\frac{\left.\operatorname{det}\left(\left(B_{1}\right)_{1,1}(x)-z I_{m}\right)\right)}{\left.\operatorname{det}\left(\left(B_{2}\right)_{1,1}(x)-z I_{m}\right)\right)}
$$


for a.e. $x \in\left(x_{0}, x_{0}+a\right)$. Next, we intend to prove that

(5.65) $\left.\left.\operatorname{det}\left(\left(B_{1}\right)_{1,1}(x)-z I_{m}\right)\right)=\operatorname{det}\left(\left(B_{2}\right)_{1,1}(x)-z I_{m}\right)\right)$ for a.e. $x \in\left(x_{0}, x_{0}+a\right)$.

Given the fact that $\left(B_{j}\right)_{1,1}(x), j=1,2$, is self-adjoint, showing (5.65) is equivalent to showing that $B_{1}(x)$ and $B_{2}(x)$ are unitarily equivalent for a.e. $x \in\left(x_{0}, x_{0}+a\right)$. Arguing by contradiction, we assume that at least one pair of eigenvalues of $B_{1}(x)$ and $B_{2}(x)$ differs. Thus, fixing $x_{1} \in\left(x_{0}, x_{0}+a\right)$, let $\lambda\left(x_{1}\right)$ be an eigenvalue of $B_{1}\left(x_{1}\right)$ but not of $B_{2}\left(x_{1}\right)$. Then (5.64) implies, for all $\alpha \in \mathbb{C}^{m \times 2 m}$ satisfying (2.9), that

$$
\operatorname{det}\left(\phi_{1,1}\left(\lambda\left(x_{1}\right), x_{1}, x_{0}, \alpha\right)\right)=0 .
$$

Next, for $\lambda \in \mathbb{R}$ and $x>x_{0}$ define

$$
\begin{aligned}
N(\lambda, x, \alpha)= & \theta_{1,2}\left(\lambda, x, x_{0}, \alpha\right) \theta_{1,2}\left(\lambda, x, x_{0}, \alpha\right)^{*} \\
& +\phi_{1,2}\left(\lambda, x, x_{0}, \alpha\right) \phi_{1,2}\left(\lambda, x, x_{0}, \alpha\right)^{*} .
\end{aligned}
$$

Then, $N(\lambda, x, \alpha)$ is strictly positive definite,

$$
N(\lambda, x, \alpha)>0 .
$$

Indeed, suppose $N f=0$ for some $f \in \mathbb{C}^{m}$; then

$$
\theta_{1,2}(\lambda) \theta_{1,2}(\lambda)^{*} f+\phi_{1,2}(\lambda) \phi_{1,2}(\lambda)^{*} f=0
$$

implies

$$
\theta_{1,2}(\lambda) \theta_{1,2}(\lambda)^{*} f=0, \quad \phi_{1,2}(\lambda) \phi_{1,2}(\lambda)^{*} f=0
$$

and hence

$$
\theta_{1,2}(\lambda)^{*} f=0, \quad \phi_{1,2}(\lambda)^{*} f=0 .
$$

Thus,

$$
f=\left(\theta_{1,1}(\lambda) \phi_{1,2}(\lambda)^{*}-\phi_{1,1}(\lambda) \theta_{1,2}(\lambda)^{*}\right) f=0
$$

by (2.97), and hence $f=0$ proves (5.68). Introducing $\alpha_{0}=\left(I_{m} 0\right) \in \mathbb{C}^{m \times 2 m}$ and $\gamma=\left(\gamma_{1} \gamma_{2}\right) \in \mathbb{C}^{m \times 2 m}$ defined by

$$
\begin{aligned}
\gamma_{1}= & {\left[\theta_{1,2}\left(\lambda\left(x_{1}\right), x_{1}, x_{0}, \alpha_{0}\right) \theta_{1,2}\left(\lambda\left(x_{1}\right), x_{1}, x_{0}, \alpha_{0}\right)^{*}\right.} \\
& \left.+\phi_{1,2}\left(\lambda\left(x_{1}\right), x_{1}, x_{0}, \alpha_{0}\right) \phi_{1,2}\left(\lambda\left(x_{1}\right), x_{1}, x_{0}, \alpha_{0}\right)^{*}\right]^{-1 / 2} \theta_{1,2}\left(\lambda\left(x_{1}\right), x_{1}, x_{0}, \alpha_{0}\right), \\
5.74) & \\
\gamma_{2}= & {\left[\theta_{1,2}\left(\lambda\left(x_{1}\right), x_{1}, x_{0}, \alpha_{0}\right) \theta_{1,2}\left(\lambda\left(x_{1}\right), x_{1}, x_{0}, \alpha_{0}\right)^{*}\right.} \\
& \left.+\phi_{1,2}\left(\lambda\left(x_{1}\right), x_{1}, x_{0}, \alpha_{0}\right) \phi_{1,2}\left(\lambda\left(x_{1}\right), x_{1}, x_{0}, \alpha_{0}\right)^{*}\right]^{-1 / 2} \phi_{1,2}\left(\lambda\left(x_{1}\right), x_{1}, x_{0}, \alpha_{0}\right),
\end{aligned}
$$

one verifies that $\gamma \gamma^{*}=I_{m}$ (by (15.73) and (5.74)) and $\gamma J \gamma^{*}=0$ (by (2.95)). Thus, $\gamma$ satisfies (2.9). Next, since

$$
\phi_{1,1}\left(\lambda\left(x_{1}\right), x_{1}, x_{0}, \gamma\right)=\phi_{1,1}\left(\lambda\left(x_{1}\right), x_{1}, x_{0}, \alpha_{0}\right) \gamma_{1}^{*}-\theta_{1,1}\left(\lambda\left(x_{1}\right), x_{1}, x_{0}, \alpha_{0}\right) \gamma_{2}^{*}
$$


as a special case of (2.98), one derives

$$
\begin{aligned}
\phi_{1,1}\left(\lambda\left(x_{1}\right), x_{1}, x_{0}, \gamma\right)= & {\left[\phi_{1,1}\left(\lambda\left(x_{1}\right), x_{1}, x_{0}, \alpha_{0}\right) \theta_{1,2}\left(\lambda\left(x_{1}\right), x_{1}, x_{0}, \alpha_{0}\right)^{*}\right.} \\
& \left.-\theta_{1,1}\left(\lambda\left(x_{1}\right), x_{1}, x_{0}, \alpha_{0}\right) \phi_{1,2}\left(\lambda\left(x_{1}\right), x_{1}, x_{0}, \alpha_{0}\right)^{*}\right] \\
& \times\left[\theta_{1,2}\left(\lambda\left(x_{1}\right), x_{1}, x_{0}, \alpha_{0}\right) \theta_{1,2}\left(\lambda\left(x_{1}\right), x_{1}, x_{0}, \alpha_{0}\right)^{*}\right. \\
& \left.+\phi_{1,2}\left(\lambda\left(x_{1}\right), x_{1}, x_{0}, \alpha_{0}\right) \phi_{1,2}\left(\lambda\left(x_{1}\right), x_{1}, x_{0}, \alpha_{0}\right)^{*}\right]^{-1 / 2} \\
= & -\left[\theta_{1,2}\left(\lambda\left(x_{1}\right), x_{1}, x_{0}, \alpha_{0}\right) \theta_{1,2}\left(\lambda\left(x_{1}\right), x_{1}, x_{0}, \alpha_{0}\right)^{*}\right. \\
& \left.+\phi_{1,2}\left(\lambda\left(x_{1}\right), x_{1}, x_{0}, \alpha_{0}\right) \phi_{1,2}\left(\lambda\left(x_{1}\right), x_{1}, x_{0}, \alpha_{0}\right)^{*}\right]^{-1 / 2}<0 .
\end{aligned}
$$

using (2.97). This contradiction to (5.66) proves (5.65). Hence, for $\lambda \in \mathbb{R}$ and for a.e. $x \in\left(x_{0}, x_{0}+a\right)$,

$$
\left|\operatorname{det}\left(\phi_{1,1}\left(\lambda, x, x_{0}, \alpha\right)\right)\right|=\left|\operatorname{det}\left(\phi_{2,1}\left(\lambda, x, x_{0}, \alpha\right)\right)\right|,
$$

by (5.64). Equation (5.77) implies that for a.e. $x_{1} \in\left(x_{0}, x_{0}+a\right)$, the family of Dirac operators $D_{+}\left(\alpha, \alpha_{0}\right)$ in $L^{2}\left(\left[x_{0}, x_{1}\right]\right)^{2 m}$, defined by

$$
\begin{aligned}
& D_{+}\left(\alpha, \alpha_{0}\right)= J \frac{d}{d x}-B, \\
& \operatorname{dom}\left(D_{+}\left(\alpha, \alpha_{0}\right)\right)=\left\{\phi \in L^{2}\left(\left[x_{0}, x_{1}\right]\right)^{2 m} \mid \phi \in \mathrm{AC}\left(\left[x_{0}, x_{1}\right]\right)^{2 m} ;\right. \\
&\left.\quad \alpha \phi\left(x_{0}\right)=0, \alpha_{0} \phi\left(x_{1}\right)=0 ;\left(J \phi^{\prime}-B \phi\right) \in L^{2}\left(\left[x_{0}, x_{1}\right]\right)^{2 m}\right\},
\end{aligned}
$$

with $\alpha_{0}=\left(I_{m} 0\right)$, have identical spectra for all boundary data $\alpha \in \mathbb{C}^{m \times 2 m}$ satisfying (2.9). Hence, assuming $B_{j} \in L^{\infty}\left(\left[x_{0}, x_{0}+a\right]\right)^{2 m \times 2 m}, j=1,2$, one can apply Theorem 2.3 of Malamud 89] and obtain [5.34).

We should note that Malamud's Theorem 2.3 in 89 only requires the equality of $m^{2}+1$ spectra (associated with linearly independent boundary data indexed by $\left.\alpha \in \mathbb{C}^{m \times 2 m}\right)$ in order to conclude (5.34).

There is no particular significance of the rays $\rho_{\ell}, \ell=1,2$, in Theorem 5.3. Any non-selfintersecting Jordan arc that tends to infinity in the sectors $\varepsilon \leq \arg (z) \leq$ $(\pi / 2)-\varepsilon$ and $(\pi / 2)+\varepsilon \leq \arg (z) \leq \pi-\varepsilon$ for some $0<\varepsilon<\pi / 4$ will do.

Remark 5.4. We were not able to prove (5.34) directly from (5.53)-(5.56), without resorting to the arguments involving (5.77) and (5.78). To conclude the proof according to the Borg-type Theorem 2.3 of Malamud [89] (cf. also Theorem 4 in [90]) requires the introduction of the extra hypothesis $B_{j} \in L^{\infty}\left(\left[x_{0}, x_{0}+a\right]\right)^{2 m \times 2 m}, j=$ 1,2 , in the matrix context $m>1$, since the construction of transformation operators for Dirac-type systems, to date, uses such an additional hypothesis on $B$. This extra hypothesis is clearly superfluous in the case $m=1$. Obviously, one conjectures that this extra hypothesis on $B_{j}$ should also be redundant in Theorem 5.3 but this appears to require nontrivial future efforts. In this context it might be interesting to note that the higher-order expansions (5.8)-(5.10) do not determine $B$ uniquely. An explicit analysis shows that while they do determine $B_{1,2}$, they only determine $B_{1,1}^{2}$, not $B_{1,1}$ itself. So that approach does not aid in proving (5.34) (besides, it would require the additional hypotheses (5.6) on $B$ ).

The corresponding local uniqueness result in terms of $M\left(z, x_{0}, \alpha\right)$ then reads as follows.

Theorem 5.5. Fix $x_{0} \in \mathbb{R}$ and suppose $A_{j}=I_{2 m}, B_{j} \in L_{\mathrm{loc}}^{1}(\mathbb{R})^{2 m \times 2 m}$, and $B_{j}=B_{j}^{*}$ a.e. on $\mathbb{R}, j=1,2$. Suppose also that $B_{j}$ is in the normal form given in 
(5.1) a.e. on $\left(x_{0}, \infty\right), j=1,2$. Denote by $M_{j}\left(z, x_{0}, \alpha\right)$ the unique Weyl-Titchmarsh matrices (2.77) corresponding to the Dirac-type operators $D_{j}, j=1,2$, in (2.76). Then,

$$
\text { if for some } a>0, B_{1}(x)=B_{2}(x) \text { for a.e. } x \in\left(x_{0}-a, x_{0}+a\right) \text {, }
$$

one obtains

$$
\left\|M_{1}\left(z, x_{0}, \alpha\right)-M_{2}\left(z, x_{0}, \alpha\right)\right\|_{\mathbb{C}^{2 m \times 2 m}} \underset{\substack{|z| \rightarrow \infty \\ z \in \rho_{+}}}{=} O\left(e^{-2 \operatorname{Im}(z) a}\right)
$$

along any ray $\rho_{+} \subset \mathbb{C}_{+}$with $0<\arg (z)<\pi$ and for all $\alpha \in \mathbb{C}^{m \times 2 m}$ satisfying (2.9). Conversely, fix an $\hat{\alpha} \in \mathbb{C}^{m \times 2 m}$ satisfying (2.9), and if $m>1$, assume in addition that $B_{j} \in L^{\infty}\left(\left[x_{0}-a, x_{0}+a\right]\right)^{2 m \times 2 m}, j=1,2$. Moreover, suppose that for all $\varepsilon>0$,

$$
\left\|M_{1}\left(z, x_{0}, \hat{\alpha}_{1}\right)-M_{2}\left(z, x_{0}, \hat{\alpha}_{1}\right)\right\|_{\mathbb{C}^{2 m \times 2 m}} \underset{\substack{|z| \rightarrow \infty \\ z \in \rho_{+, \ell}}}{=} O\left(e^{-2 \operatorname{Im}(z)(a-\varepsilon)}\right), \quad \ell=1,2,
$$

along a ray $\rho_{+, 1} \subset \mathbb{C}_{+}$with $0<\arg (z)<\pi / 2$ and along a ray $\rho_{+, 2} \subset \mathbb{C}_{+}$with $\pi / 2<\arg (z)<\pi$. Then

$$
B_{1}(x)=B_{2}(x) \quad \text { for a.e. } x \in\left[x_{0}-a, x_{0}+a\right] \text {. }
$$

Proof. (5.80) is proved by combining (2.78) with (5.31), (5.32), and then (5.82) follows by combining (2.78) with (5.33), (5.34), taking into account the asymptotic expansions

$$
M_{ \pm}\left(z, x_{0}\right) \underset{|z| \rightarrow \infty}{=} \pm i I_{m}+o(1)
$$

along any ray with $\varepsilon<\arg (z)<\pi-\varepsilon$ in the case of Dirac-type operators (cf. (3.1)).

Remark 5.6. Theorem 5.3 and Theorem 5.5 yield new global uniqueness theorems for half-line and full-line Dirac-type operators, extending the classical BorgMarchenko-type results. Indeed, if (5.33) (resp., (5.81) ) holds for all $a>0$, then (5.34) (resp. (5.82) ) holds for a.e. $x \in\left[x_{0}, \infty\right)$ (resp., for a.e. $x \in \mathbb{R}$ ).

In the case of scalar Schrödinger operators, the analog of Theorem 5.3 is due to Simon [115. An alternative proof, applicable to matrix-valued Schrödinger operators, was presented in [51] (cf. also [42]). More recently, yet another proof was found by Bennewitz 13 . (following some ideas in [16]). These results extend the classical (global) uniqueness results due to Borg [16] and Marchenko [92, 93] (cf. also 14]), which state that half-line $m$-functions uniquely determine the corresponding potential coefficient. The Dirac-type results presented in this section (especially, all local considerations) appear to be new, even in the special case $m=1$. Previous results in the Dirac case focused on global uniqueness questions only. We refer to Gasymov and Levitan [35] in the case $m=1$ and to Lesch and Malamud 82. in the matrix case $m \in \mathbb{N}$. Most recently, Alexander Sakhnovich kindly informed us that his integral representation of the Weyl-Titchmarsh matrix in [104] can be used to derive asymptotic expansions for the Weyl-Titchmarsh matrix and its associated matrix-valued spectral function, and also yields a result analogous to Theorem 5.3 (i) for a certain class of canonical systems. Moreover, in the case of skew-adjoint Dirac-type systems, similar results are discussed in [105] and applied to the nonlinear Schrödinger equation on a half-axis. 
Although not directly used in this paper, it should be pointed out that inverse monodromy problems for canonical systems have received a lot of attention (some of it very recently). The interested reader is referred to [4, [5], 66, 88, 89], 90, [110], [113] and the extensive literature cited therein. Moreover, inverse spectral theory associated with canonical systems is discussed in [97, 105, 107, 108, [110, 111], 112], 113] (see also the extensive literature cited in [42]).

\section{Trace Formulas and Borg-Type Theorems}

In our final section we derive a trace formula for $B$ and then discuss its application to Borg-type uniqueness theorems for Dirac-type operators.

Theorem 6.1. Assume Hypothesis 2.1 with $A=I_{2 m}$, and let $\alpha_{0}=\left(I_{m} 0\right) \in$ $\mathbb{C}^{m \times 2 m}$. Fix $x_{0} \in \mathbb{R}$ and suppose that for all $R>0$,

$$
\begin{aligned}
& \operatorname{esssup}_{y \in\left[x_{0}, x_{0}+R\right]}\left\|\int_{y}^{x_{0}+R} d x^{\prime} B\left(x^{\prime}\right) \exp \left(2 i z\left(x^{\prime}-y\right)\right)+\frac{1}{2 i z} B(y)\right\|_{\mathbb{C}^{2 m \times 2 m}} \\
& +\operatorname{ess~sup}_{y \in\left[x_{0}-R, x_{0}\right]}\left\|\int_{x_{0}-R}^{y} d x^{\prime} B\left(x^{\prime}\right) \exp \left(2 i z\left(x^{\prime}-y\right)\right)-\frac{1}{2 i z} B(y)\right\|_{\mathbb{C}^{2 m \times 2 m}} \\
& \underset{\substack{|z| \rightarrow \infty \\
z \in \rho_{+}}}{=} o\left(|z|^{-1}\right)
\end{aligned}
$$

along a ray $\rho_{+} \subset \mathbb{C}_{+}$. In addition, assume $B_{k, k^{\prime}} B_{\ell, \ell^{\prime}} \in L_{\text {loc }}^{1}(\mathbb{R})^{m \times m}$ for all $k, k^{\prime}, \ell, \ell^{\prime} \in\{1,2\}$. Then, with $\Upsilon\left(\lambda, x, \alpha_{0}\right)$ defined in (2.84),

$$
\begin{aligned}
& \left(\begin{array}{ll}
B_{1,1}(x)-B_{2,2}(x) & B_{1,2}(x)+B_{2,1}(x) \\
B_{1,2}(x)+B_{2,1}(x) & B_{2,2}(x)-B_{1,1}(x)
\end{array}\right) \\
& =\lim _{\substack{|z| \rightarrow \infty \\
z \in \rho_{+}}} 2 \int_{\mathbb{R}} d \lambda z^{2}(\lambda-z)^{-2} \Upsilon\left(\lambda, x, \alpha_{0}\right) \text { for a.e. } x \in \mathbb{R} .
\end{aligned}
$$

Proof. By (2.79),

$$
\frac{d}{d z} \ln \left(M\left(z, x, \alpha_{0}\right)\right)=\int_{\mathbb{R}} d \lambda(\lambda-z)^{-2} \Upsilon\left(\lambda, x, \alpha_{0}\right) .
$$

Next, suppose that $x \in \mathbb{R}$ is a left and right Lebesgue point of $B$. By (4.65), (4.66) one obtains

$$
\begin{aligned}
& \frac{d}{d z} \ln \left(M\left(z, x, \alpha_{0}\right)\right) \\
& \begin{aligned}
(6.4) & \\
\quad= & \frac{1}{|z| \rightarrow \infty}\left(\begin{array}{ll}
B_{1,1}(x+0)-B_{2,2}(x+0) & B_{1,2}(x+0)+B_{2,1}(x+0) \\
B_{1,2}(x+0)+B_{2,1}(x+0) & B_{2,2}(x+0)-B_{1,1}(x+0)
\end{array}\right) z^{-2} \\
\quad+\rho_{+} & \frac{1}{4}\left(\begin{array}{ll}
B_{1,1}(x-0)-B_{2,2}(x-0) & B_{1,2}(x-0)+B_{2,1}(x-0) \\
B_{1,2}(x-0)+B_{2,1}(x-0) & B_{2,2}(x-0)-B_{1,1}(x-0)
\end{array}\right) z^{-2}+o\left(z^{-2}\right)
\end{aligned}
\end{aligned}
$$


and hence

$$
\begin{aligned}
& \frac{1}{2}\left(\begin{array}{ll}
B_{1,1}(x+0)-B_{2,2}(x+0) & B_{1,2}(x+0)+B_{2,1}(x+0) \\
B_{1,2}(x+0)+B_{2,1}(x+0) & B_{2,2}(x+0)-B_{1,1}(x+0)
\end{array}\right) \\
+ & \frac{1}{2}\left(\begin{array}{ll}
B_{1,1}(x-0)-B_{2,2}(x-0) & B_{1,2}(x-0)+B_{2,1}(x-0) \\
B_{1,2}(x-0)+B_{2,1}(x-0) & B_{2,2}(x-0)-B_{1,1}(x-0)
\end{array}\right) \\
= & \lim _{\substack{|z| \rightarrow \infty \\
z \in \rho_{+}}} 2 \int_{\mathbb{R}} d \lambda z^{2}(\lambda-z)^{-2} \Upsilon\left(\lambda, x, \alpha_{0}\right) .
\end{aligned}
$$

Since a.e. $x \in \mathbb{R}$ is a Lebesgue point of $B$, one concludes (6.2).

In the case $m=1$, a trace formula for Dirac-type operators, using Krein spectral shift functions and exponential representations of Herglotz functions, was discussed in 117. This circle of ideas was first introduced in connection with trace formulas of Schrödinger operators in [49] (see also [39], 40], 102], [103] in the scalar case $m=1$. The corresponding case of trace formulas for matrix-valued Schrödinger operators was introduced in [38] (see also [21]).

Analogous trace formulas can be drived for all higher-order coefficients $M_{k}\left(x, \alpha_{0}\right)$ in (4.65) (see, e.g., [40 in connection with scalar Schrödinger operators).

A comparison of the trace formula (3.20) in 21] for Schrödinger operators with its Dirac-type counterpart (6.2) reveals characteristic differences. While in the Schrödinger case the trace formula directly involves the potential coefficient $Q(x)$, $M_{1}\left(x, \alpha_{0}\right)$ differs markedly from a constant multiple of $B(x)$, and consequently, the Dirac-type trace formula (6.2) does not directly involve $B(x)$ but certain linear combinations of $B_{j, k}(x)$. This is related to the fact that $M\left(z, x_{0}, \alpha_{0}\right)$ (or equivalently, $\left.\Upsilon\left(\lambda, x_{0}, \alpha_{0}\right)\right)$, in general, does not uniquely determine $B$ a.e. In fact, there exists a typical ambiguity concerning the coefficients of $D$ related to unitary gauge transformations of $D$. In the case $m=1$, this ambiguity is well known and discussed, e.g., in [35], 85, Sect. I.10], 86, Ch. 7]. These gauge transformations leave the spectrum of $D$ invariant and suggest that we focus our attention on certain normal forms of $D$ in connection with inverse spectral problems for Dirac-type operators.

Lemma 6.2. Assume Hypothesis 2.17. Then $D=J \frac{d}{d x}-B$ is unitarily equivalent to $\widetilde{D}$, where $\widetilde{D}$ in $L^{2}(\mathbb{R})^{2 m}$ is of the normal form

$$
\widetilde{D}=J \frac{d}{d x}-\widetilde{B}=\left(\begin{array}{cc}
-\widetilde{B}_{1,1} & -I_{m} \frac{d}{d x}-\widetilde{B}_{1,2} \\
I_{m} \frac{d}{d x}-\widetilde{B}_{1,2} & \widetilde{B}_{1,1}
\end{array}\right) .
$$

Here $\widetilde{B}=\widetilde{B}^{*}$ a.e. and

$$
\begin{aligned}
& \widetilde{B}_{1,1}=-(1 / 2) \operatorname{Im}\left(U_{1,1}^{-1}\left[\left(B_{1,2}+B_{2,1}\right)-i\left(B_{1,1}-B_{2,2}\right)\right] U_{2,2}\right)=\widetilde{B}_{1,1}^{*}, \\
& \widetilde{B}_{1,2}=(1 / 2) \operatorname{Re}\left(U_{1,1}^{-1}\left[\left(B_{1,2}+B_{2,1}\right)-i\left(B_{1,1}-B_{2,2}\right)\right] U_{2,2}\right)=\widetilde{B}_{1,2}^{*},
\end{aligned}
$$

with $U_{j, j} \in \mathbb{C}^{m \times m}, j=1,2$, satisfying the first-order system of ordinary differential equations:

$$
i U_{j, j}^{\prime}(x)=-(1 / 2)\left((-1)^{j}\left(B_{1,1}(x)+B_{2,2}(x)\right)+i\left(B_{1,2}(x)-B_{2,1}(x)\right)\right) U_{j, j}(x),
$$

Proof. We start with the unitary transformation $V$ in $L^{2}(\mathbb{R})^{2 m}$ defined by

$$
V=\frac{1}{\sqrt{2}}\left(\begin{array}{cc}
i I_{m} & I_{m} \\
I_{m} & i I_{m}
\end{array}\right), \quad V^{-1}=\frac{1}{\sqrt{2}}\left(\begin{array}{cc}
-i I_{m} & I_{m} \\
I_{m} & -i I_{m}
\end{array}\right)
$$


which maps $D$ to $D_{1}$, where

$$
\begin{aligned}
D_{1}= & V^{-1} D V=i\left(\begin{array}{cc}
I_{m} \frac{d}{d x} & 0 \\
0 & -I_{m} \frac{d}{d x}
\end{array}\right) \\
& -\frac{1}{2}\left(\begin{array}{ll}
B_{1,1}+B_{2,2}-i\left(B_{1,2}-B_{2,1}\right) & B_{1,2}+B_{2,1}-i\left(B_{1,1}-B_{2,2}\right) \\
B_{1,2}+B_{2,1}+i\left(B_{1,1}-B_{2,2}\right) & B_{1,1}+B_{2,2}+i\left(B_{1,2}-B_{2,1}\right)
\end{array}\right) .
\end{aligned}
$$

Next, we introduce the unitary operator $U$ in $L^{2}(\mathbb{R})^{2 m}$ defined by

$$
U=\left(\begin{array}{cc}
U_{1,1} & 0 \\
0 & U_{2,2}
\end{array}\right)
$$

where the unitary $m \times m$ matrices $U_{j, j} \in \mathbb{C}^{m \times m}$ are solutions of the first-order system (6.9). Since by hypothesis $B_{j, k} \in L_{\text {loc }}^{1}(\mathbb{R})^{m \times m}, 1 \leq j, k \leq 2$, the solutions of equation (6.9) are well-defined and $U_{j, j} \in \mathrm{AC}_{\text {loc }}(\mathbb{R})^{m \times m}, j=1,2$. One computes

$$
\widehat{D}=U^{-1} D_{1} U=\left(\begin{array}{cc}
i I_{m} \frac{d}{d x} & -\widehat{B}_{1,2} \\
-\widehat{B}_{1,2}^{*} & -i I_{m} \frac{d}{d x}
\end{array}\right),
$$

where $\widehat{B}_{1,2} \in L_{\text {loc }}^{1}(\mathbb{R})^{m \times m}$ and

$$
\widehat{B}_{1,2}(x)=(1 / 2) U_{1,1}^{-1}(x)\left(B_{1,2}(x)+B_{2,1}(x)-i\left(B_{1,1}(x)-B_{2,2}(x)\right) U_{2,2}(x) .\right.
$$

Finally, defining $\widetilde{D}=V \widehat{D} V^{-1}$, one arrives at (6.6)

Thus, unitary invariants of $D$ (such as the spectrum of $D$, denoted by $\operatorname{spec}(D)$, and its multiplicity) cannot determine $B$ in general, but at best determine a potential matrix of the type (normal form) $\widetilde{B}$ in (6.6). A further restriction on the solvability of inverse spectral problems for Dirac-type operators is mentioned in the following result.

Lemma 6.3. Assume Hypotheses 2.17 and let $\omega=\omega^{*} \in \mathbb{C}^{m \times m}$ be a constant selfadjoint $m \times m$ matrix. Then $D=J \frac{d}{d x}-B$ is unitarily equivalent to $\widetilde{D}_{\omega}$ in $L^{2}(\mathbb{R})^{2 m}$, where

$$
\widetilde{D}_{\omega}=J \frac{d}{d x}-\widetilde{B}_{\omega}=\left(\begin{array}{cc}
-\widetilde{B}_{\omega, 1,1} & -I_{m} \frac{d}{d x}-\widetilde{B}_{\omega, 1,2} \\
I_{m} \frac{d}{d x}-\widetilde{B}_{\omega, 1,2} & \widetilde{B}_{\omega, 1,1}
\end{array}\right),
$$

with

$$
\widetilde{B}_{\omega, 1,1}=-(1 / 2) \operatorname{Im}\left(e^{i \omega} U_{1,1}^{-1}\left[\left(B_{1,2}+B_{2,1}\right)-i\left(B_{1,1}-B_{2,2}\right)\right] U_{2,2} e^{i \omega}\right)=\widetilde{B}_{\omega, 1,1}^{*},
$$

$$
\widetilde{B}_{\omega, 1,2}=(1 / 2) \operatorname{Re}\left(e^{i \omega} U_{1,1}^{-1}\left[\left(B_{1,2}+B_{2,1}\right)-i\left(B_{1,1}-B_{2,2}\right)\right] U_{2,2} e^{i \omega}\right)=\widetilde{B}_{\omega, 1,2}^{*},
$$

and with $U_{j, j}, j=1,2$, satisfying the first-order system (6.9).

Proof. Define

$$
U_{\omega}=\left(\begin{array}{cc}
e^{i \omega} & 0 \\
0 & e^{-i \omega}
\end{array}\right) .
$$

Using the notation employed in the proof of Lemma 6.2, one verifies that

$$
\widetilde{D}_{\omega}=V U_{\omega}(V U)^{-1} D V U\left(V U_{\omega}\right)^{-1} \text {. }
$$


In particular, choosing $\omega=(\pi / 2) I_{m}$ effects the sign change $\widetilde{B} \rightarrow-\widetilde{B}$, with $\widetilde{B}$ given by (6.7), (6.8).

For detailed discussions of various normal forms for Dirac-type operators we refer to [35], [60], [85, Ch. 9], [86, Ch. 7] in the case $m=1$ and to [34], 82], [89], [94, pp. 193-195], [96 in the general matrix-valued case. Perhaps it should be noted that if $D$ is in its normal form $\widetilde{D}$ as in (6.6), $\widetilde{D}^{2}$ turns into a $2 m \times 2 m$ matrix-valued Schrödinger operator under appropriate regularity assumptions on $\widetilde{B}$. Details on this fact and the relation between the $M$-matrices of $\widetilde{D}$ and $\widetilde{D}^{2}$ can be found in Section 3 of 42 .

Next, we turn to Borg-type theorems, one of the principal topics of this paper. In 1946 Borg [15] proved, among a variety of other inverse spectral theorems, the following result.

Theorem $6.4([15])$. Assume $q \in L_{\mathrm{loc}}^{2}(\mathbb{R})$ to be real-valued and periodic, and let

$$
h=-d^{2} / d x^{2}+q
$$

be the associated self-adjoint Schrödinger operator in $L^{2}(\mathbb{R})$. Moreover, suppose that $\operatorname{spec}(h)=\left[e_{0}, \infty\right)$ for some $e_{0} \in \mathbb{R}$. Then

$$
q(x)=e_{0} \quad \text { for a.e. } x \in \mathbb{R} .
$$

The analog of Theorem 6.4 for Dirac-type operators (in the case $m=1$ ) was proven by Giachetti and Johnson [54] in 1984 (see also [36], [37, [48 in the special case where $p$ is constant, and [56] in the case where $p, q \in L^{2}(\mathbb{R})$ are real-valued and periodic).

Theorem 6.5 ([54]). Assume $p, q \in L^{\infty}(\mathbb{R})$ to be real-valued and periodic, and let

$$
d=\left(\begin{array}{cc}
-p & -\frac{d}{d x}-q \\
\frac{d}{d x}-q & p
\end{array}\right)
$$

be the associated self-adjoint Dirac-type operator in $L^{2}(\mathbb{R})^{2}$. Moreover, suppose that $\operatorname{spec}(d)=\mathbb{R}$. Then

$$
p(x)=q(x)=0 \quad \text { for a.e. } x \in \mathbb{R} .
$$

Traditionally, uniqueness results such as Theorems 6.4 and 6.5 are called Borgtype theorems. (However, this terminology is not uniquely adopted and hence a bit unfortunate. Indeed, inverse spectral results on finite intervals recovering the potential coefficient(s) from several spectra were also pioneered by Borg in his celebrated paper [15], and hence are also coined Borg-type theorems in the literature; see, e.g., [87, 89], [90].)

A quick and natural proof of Theorem 6.4 based on a trace formula for $q$, was presented in [21. This strategy of proof was then applied to the case of matrix-valued Schrödinger operators, and the corresponding matrix-valued analog of Theorem 6.4 was also proved in [21] along these lines. A closer examination of the proof of Theorem 6.4 shows that periodicity of $q$ is not the crucial element in the proof of the uniqueness result (6.20). The key ingredient (besides $\operatorname{spec}(h)=\left[e_{0}, \infty\right)$ ) is clearly the fact that for all $x \in \mathbb{R}$,

$$
\xi(\lambda, x)=1 / 2 \quad \text { for a.e. } \lambda \in \operatorname{spec}_{\mathrm{ess}}(h)
$$

$\left(\operatorname{spec}_{\text {ess }}(\cdot)\right.$ is the essential spectrum), where $\xi(\cdot, x)$ is defined by

$$
\xi(\lambda, x)=\lim _{\varepsilon \rightarrow 0} \pi^{-1} \operatorname{Im}(\ln (g(\lambda+i \varepsilon, x))) \quad \text { for a.e. } \lambda \in \mathbb{R},
$$


and $g(z, x)$ denotes Green's function (i.e., the integral kernel of the resolvent) of $h$ on the diagonal,

$$
g(z, x)=(h-z)^{-1}(x, x) .
$$

Completely analogous considerations apply to the Dirac-type case.

Real-valued periodic potentials are known to satisfy (6.23), but so are certain classes of real-valued quasi-periodic and almost-periodic potentials $q$ (see, e.g., [23], [24], [26], 71], 73], 76], 77], 78], [79, [116]). In particular, the class of real-valued algebro-geometric finite-gap potentials $q$ (a subclass of the set of real-valued quasiperiodic potentials) is a prime example satisfying (6.23) without necessarily being periodic. Traditionally, potentials $q$ satisfying (6.23) are called reflectionless (see [24], 26], 78], 116]). Again the analogous notions apply to the Dirac-type case (cf., e.g., [23], [54], [72]).

Taking this circle of ideas as the point of departure for our derivation of Borg-type results for Dirac-type operators, we now use the reflectionless situation described in (6.23), or, more precisely, its proper analog for Dirac-type systems, as the model for the subsequent definition.

Definition 6.6. Assume Hypothesis 2.1 with $A=I_{2 m}$, and let $\alpha_{0}=\left(\begin{array}{ll}I_{m} & 0\end{array}\right) \in$ $\mathbb{C}^{m \times 2 m}$. Then $B$ is called reflectionless if, for all $x \in \mathbb{R}$,

$$
\Upsilon\left(\lambda, x, \alpha_{0}\right)=(1 / 2) I_{2 m} \quad \text { for a.e. } \lambda \in \operatorname{spec}_{\text {ess }}(D) .
$$

Since hardly any confusion can arise, we will also call the Dirac-type operator $D$ reflectionless if (6.26) is satisfied.

Given Definition 6.6, we turn to a Borg-type uniqueness theorem and formulate the analog of Theorem 6.4 for (reflectionless) Dirac-type operators.

Theorem 6.7. Assume Hypothesis [2.1 with $A=I_{2 m}$, and let $\alpha_{0}=\left(I_{m} 0\right) \in$ $\mathbb{C}^{m \times 2 m}$. If for all $x \in \mathbb{R}, \Upsilon\left(\lambda, x, \alpha_{0}\right)=C$ is a constant $2 m \times 2 m$ matrix for a.e. $\lambda \in \mathbb{R}$, especially, if $B$ is reflectionless and $\operatorname{spec}(D)=\mathbb{R}$, then

$$
B_{1,1}(x)=B_{2,2}(x), \quad B_{1,2}(x)=-B_{2,1}(x) \quad \text { for a.e. } x \in \mathbb{R} .
$$

In particular, if $D$ is assumed to be in its normal form (6.6), that is, of the type $\widetilde{D}=J \frac{d}{d x}-\widetilde{B}$, then

$$
\widetilde{B}(x)=0 \quad \text { for a.e. } x \in \mathbb{R} .
$$

Proof. The fact that $\int_{\mathbb{R}} d \lambda(\lambda-z)^{-2}=0$ for all $z \in \mathbb{C} \backslash \mathbb{R}$, that a.e. $x \in \mathbb{R}$ is a Lebesgue point of $B$, and the trace formula (6.2), imply 6.27). Together with Lemma 6.2, this yields (6.28).

The analog of Theorem 6.7 for matrix-valued Schrödinger operators was recently proved in [21].

In the remainder of the section we will show that the case of periodic $B$ is covered by Theorem 6.7 under appropriate uniform multiplicity assumptions on $\operatorname{spec}(D)$. In order to handle Floquet-theoretic aspects of periodic Dirac-type operators $D$, we adopt the following assumptions until the end of this section.

Hypothesis 6.8. In addition to Hypothesis 2.1 assume $A=I_{2 m}$ and suppose that $B$ is periodic, that is, there is an $\omega>0$ such that $B(x+\omega)=B(x)$ for a.e. $x \in \mathbb{R}$.

The following result has been proven in [21, Theorem 4.6]. 
Theorem 6.9 ([21], Theorem 4.6). Assume Hypothesis 6.8 and let $\alpha_{0}=\left(I_{m} 0\right) \in$ $\mathbb{C}^{2 m \times m}$. If $D$ has uniform spectral multiplicity $2 m$, then for all $x \in \mathbb{R}$ and all $\lambda \in \operatorname{spec}(D)^{\circ}$,

$$
M_{+}\left(\lambda+i 0, x, \alpha_{0}\right)=M_{-}\left(\lambda+i 0, x, \alpha_{0}\right)^{*}=M_{-}\left(\lambda-i 0, x, \alpha_{0}\right) .
$$

In particular, $M_{-}\left(z, x, \alpha_{0}\right)$ is the analytic continuation of $M_{+}\left(z, x, \alpha_{0}\right)$ (and vice versa) through $\operatorname{spec}(D)^{\circ}$.

Here $A^{\circ}$ denotes the open interior of a set $A \subseteq \mathbb{R}$.

Strictly speaking, Theorem 4.6 in [21] was proved for matrix-valued Schrödinger operators. But the proof extends line by line to the corresponding Dirac-type situation and was predominantly formulated in terms of Hamiltonian systems notation (rather than Schrödinger operator specifics) in order to be applicable to the present context. In particular, the spectrum, $\operatorname{spec}(H)$, of the Schrödinger operator $H$ should be replaced by that of $D$, the point spectrum, $\operatorname{spec}_{p}\left(H_{x_{0}}^{D}\right)$, of the Dirichlet Schrödinger operator $H_{x_{0}}^{D}$ with a Dirichlet boundary condition at the point $x_{0}$ should simply be replaced by the set $\left\{\lambda \in \mathbb{R} \mid \operatorname{det}\left(\phi_{1}\left(\lambda, x_{0}+\omega, x_{0}, \alpha_{0}\right)\right)=0\right\}$, etc.

Theorem 6.10. Suppose Hypothesis 6.8 and let $\alpha_{0}=\left(I_{m} 0\right) \in \mathbb{C}^{2 m \times m}$. If $D$ has uniform spectral multiplicity $2 m$, then $D$ is reflectionless and, for all $x \in \mathbb{R}$ and all $\lambda \in \operatorname{spec}(D)^{\circ}$,

$$
\Upsilon\left(\lambda, x, \alpha_{0}\right)=(1 / 2) I_{2 m} .
$$

Proof. This is clear from (2.78) and 6.29), which imply

$$
M\left(\lambda+i 0, x, \alpha_{0}\right)=-M\left(\lambda+i 0, x, \alpha_{0}\right)^{*} .
$$

Theorems 6.9 and 6.10 extend to more general situations (not necessarily periodic ones), as is clear from the corresponding results in [23], [54], [43], 76], 777, [78,

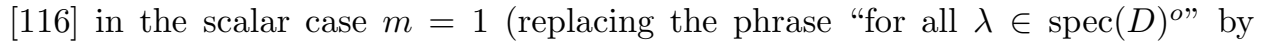

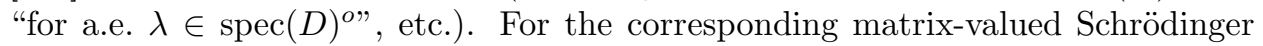
operator case we refer to [79].

Corollary 6.11. Assume Hypothesis 6.8. If D has uniform spectral multiplicity $2 m$ and $\operatorname{spec}(D)=\mathbb{R}$, then

$$
B_{1,1}(x)=B_{2,2}(x), \quad B_{1,2}(x)=-B_{2,1}(x) \quad \text { for a.e. } x \in \mathbb{R} .
$$

In particular, if $D$ is assumed to be in its normal form $\widetilde{D}=J \frac{d}{d x}-\widetilde{B}$, with $\widetilde{B}$ given by (6.6), then

$$
\widetilde{B}(x)=0 \quad \text { for a.e. } x \in \mathbb{R} .
$$

Remark 6.12. The assumption of uniform (maximal) spectral multiplicity $2 m$ in Corollary 6.11 is an essential one. Otherwise, one can easily construct nonconstant potentials $B$ such that the associated operator $D$ has overlapping band spectra and hence spectrum the whole real line. Also self-adjointness of $B$ is crucial for Corollary 6.11] to hold (cf. the corresponding discussion in Remark 4.2 of [21] in the context of Schrödinger operators).

The analog of Corollary 6.11 for periodic matrix-valued Schrödinger operators was first proved by Després [27] and recently rederived using such a trace formula approach in [21]. 
We note that all results presented in this paper also apply to matrix-valued finite-difference Hamiltonian systems. We refer the reader to [22] in this direction.

Finally, Borg-type uniqueness theorems for Hamiltonian systems are just a beginning. There is a natural extension of Borg's Theorem 6.4 to self-adjoint periodic Schrödinger, respectively, Dirac-type operators with one gap, respectively, two gaps in their spectrum. In the case of (scalar) Schrödinger operators, such an extension is due to Hochstadt [70] and the resulting potential $q$ becomes twice the elliptic Weierstrass function. In the case of Dirac-type operators (with $m=1$ and vanishing diagonal coefficients in $B$ ) such an extension involving elliptic functions can be found in [36], 37], [48] (see also [53]). Extensions to matrix-valued versions (i.e., for $m \geq 2$ ) are currently under active investigation.

Acknowledgements. We would like to thank Suzanne Collier, Helge Holden, Konstantin Makarov, Fedor Rofe-Beketov, Alexei Rybkin, Lev Sakhnovich, and Barry Simon for helpful discussions and many hints regarding the literature, and especially, Don Hinton, Boris Levitan, Mark Malamud, and Alexander Sakhnovich for repeated correspondence on various parts of the material in this paper.

S. C. would like to thank the Mathematics Department of the University of Missouri-Columbia for the warm hospitality extended to him during his 2000/2001 sabbatical, when this work was completed.

\section{REFERENCES}

[1] L. Amour, Inverse spectral theory for the AKNS system with separated boundary conditions, Inverse Problems 9, 507-523 (1993). MR 94i:34034

[2] L. Amour and J.-C. Guillot, Isospectral sets for AKNS systems on the unit interval with generalized periodic boundary conditions, Geom. Funct. Anal. 6, 1-27 (1996). MR 96k:34184

[3] N. Aronszajn and W. F. Donoghue, On exponential representations of analytic functions in the upper half-plane with positive imaginary part, J. Analyse Math. 5, 321-388 (1956-57).

[4] D. Z. Arov and H. Dym, J-inner matrix functions, interpolation and inverse problems for canonical systems, I: foundations, Integral Equations Operator Theory 29, 373-454 (1997). MR 99c: 47011

[5] D. Z. Arov and H. Dym, J-inner matrix functions, interpolation and inverse problems for canonical systems,II: the inverse monodromy problem, Integral Equations Operator Theory 36, 11-70 (2000). MR 2001g:34123

[6] D. Z. Arov and H. Dym, J-inner matrix functions, interpolation and inverse problems for canonical systems, III: more on the inverse monodromy problem, Integral Equations Operator Theory 36, 127-181 (2000). MR 2001i:34140

[7] N. Asano and Y. Kato, Algebraic and Spectral Methods for Nonlinear Wave Equations, Longman, New York, 1990. MR 92d:35001

[8] F. V. Atkinson, Discrete and Continuous Boundary Problems, Academic Press, New York, 1964. MR 31:416

[9] F. V. Atkinson, On the location of Weyl circles, Proc. Roy. Soc. Edinburgh A 88, 345-356 (1981). MR 83a:34023

[10] F. V. Atkinson, On the asymptotic behaviour of the Titchmarsh-Weyl m-coefficient and the spectral function for scalar second-order differential expressions, Lecure Notes in Mathematics, Vol. 964, Springer-Verlag, Berlin, 1982, pp. 1-27. MR 85b:34022

[11] F. V. Atkinson, On the order of magnitude of Titchmarsh-Weyl functions, Diff. Integral Eqs. 1, 79-96 (1988). MR 89d:34043

[12] F. V. Atkinson, Asymptotics of the Titchmarsh-Weyl function in the matrix case, unpublished manuscript.

[13] C. Bennewitz, A proof of the local Borg-Marchenko theorem, Comm. Math. Phys. 218, 131132 (2001). MR 2001m:34035

[14] C. Bennewitz, A Paley-Wiener theorem with applications to inverse spectral theory, preprint, University of Lund, Sweden, 2000. 
[15] G. Borg, Eine Umkehrung der Sturm-Liouvilleschen Eigenwertaufgabe, Acta Math. 78, 1-96 (1946). MR 7:382d

[16] G. Borg, Uniqueness theorems in the spectral theory of $y^{\prime \prime}+(\lambda-q(x)) y=0$, Proc. 11th Scandinavian Congress of Mathematicians, Johan Grundt Tanums Forlag, Oslo, 1952, pp. 276-287. MR 15:315a

[17] R. W. Carey, A unitary invariant for pairs of self-adjoint operators, J. reine angew. Math. 283, 294-312 (1976). MR 54:3454

[18] I. Cherednik, Basic Methods of Soliton Theory, World Scientific, Singapore, 1996. MR 97m:58090

[19] S. L. Clark, On the absolutely continuous spectrum of a vector-matrix Dirac system, Proc. Roy. Soc. Edinburgh 124A, 253-262 (1994). MR 95c:34142

[20] S. Clark and F. Gesztesy, Weyl-Titchmarsh $M$-function asymptotics for matrix-valued Schrödinger operators, Proc. London Math. Soc. 82, 701-724 (2001). MR 2002c:34144]

[21] S. Clark, F. Gesztesy, H. Holden, and B. M. Levitan, Borg-type theorems for matrix-valued Schrödinger operators, J. Diff. Eqs. 167, 181-210 (2000). MR 2002d:34019

[22] S. Clark, F. Gesztesy, and W. Renger, Trace formulas and Borg-type theorems for finite difference operators, in preparation.

[23] C. De Concini and R. A. Johnson, The algebraic-geometric AKNS potentials, Ergodic Theory and Dynam. Systems 7, 1-24 (1987). MR 88f:58065

[24] W. Craig, The trace formula for Schrödinger operators on the line, Commun. Math. Phys. 126, 379-407 (1989). MR 90m:47063

[25] Ju. L. Daleckii and M. G. Krein, Stability of Solutions of Differential Equations in Banach Spaces, Amer. Math. Soc., Providence, RI, 1974. MR 50:5126

[26] P. Deift and B. Simon, Almost periodic Schrödinger operators III. The absolutely continuous spectrum in one dimension, Commun. Math. Phys. 90, 389-411 (1983). MR 85i:30009b

[27] B. Després, The Borg theorem for the vectorial Hill's equation, Inverse Probl. 11, 97-121 (1995). MR 96a:34158

[28] L. A. Dickey, Soliton Equations and Hamiltonian Systems, World Scientific, Singapore, 1991. MR 93d:58067

[29] B. A. Dubrovin, Completely integrable Hamiltonian systems associated with matrix operators and Abelian varieties, Funct. Anal. Appl. 11, 265-277 (1977). MR 58:31219

[30] B. A. Dubrovin, Matrix finite-zone operators, Revs. Sci. Tech. 23, 20-50 (1983). MR 86a:58041

[31] W. N. Everitt, On a property of the m-coefficient of a second-order linear differential equation, J. London Math. Soc. (2), 4, 443-457 (1972). MR 45:7156

[32] W. N. Everitt and S. G. Halvorsen, On the asymptotic form of the Titchmarsh-Weyl mcoefficient, Applicable Anal. 8, 153-169 (1978). MR 80f:34019

[33] W. N. Everitt, D. B. Hinton, and J. K. Shaw, The asymptotic form of the Titchmarsh-Weyl coefficient for Dirac systems, J. London Math. Soc. (2), 27, 465-476 (1983). MR 86b:34036

[34] M. G. Gasymov, The inverse scattering problem for a system of Dirac equations of order $2 n$, Trans. Moscow Math. Soc. 19, 41-119 (1968). MR 39:2418

[35] M. G. Gasymov and B. M. Levitan, The inverse problem for a Dirac system, Sov. Math. Dokl. 7, 495-499 (1966). MR 33:2859

[36] F. Gesztesy, Some application of commutation methods, in "Schrödinger Operators", H. Holden and A. Jensen (eds.), Lecture Notes in Physics, Vol. 345, Springer, Berlin, 1989, p. 1-25. MR 91g:58246

[37] F. Gesztesy, On the modified Korteweg-deVries equation, in "Differential Equations with Applications in Biology, Physics, and Engineering", J. A. Goldstein, F. Kappel, and W. Schappacher (eds.), M. Dekker, New York, 1991, pp. 139-183. MR 93i:35123

[38] F. Gesztesy and H. Holden, On trace formulas for Schrödinger-type operators, in Multiparticle Quantum Scattering with Applications to Nuclear, Atomic and Molecular Physics, D. G. Truhlar and B. Simon (eds.), Springer, New York, 1997, pp. 121-145. MR 98m:34172

[39] F. Gesztesy, H. Holden, and B. Simon, Absolute summability of the trace relation for certain Schrödinger operators, Commun. Math. Phys. 168, 137-161 (1995). MR 96b:34110

[40] F. Gesztesy, H. Holden, B. Simon, and Z. Zhao, Higher order trace relations for Schrödinger operators, Rev. Math. Phys. 7, 893-922 (1995). MR 97d:34094

[41] F. Gesztesy, N. J. Kalton, K. A. Makarov, and E. Tsekanovskii, Some Applications of Operator-Valued Herglotz Functions, in Operator Theory, System Theory and Related Topics. 
The Moshe Livšic Anniversary Volume, D. Alpay and V. Vinnikov (eds.), Operator Theory: Advances and Applications, Vol. 123, Birkhäuser, Basel, 2001, pp. 271-321.

[42] F. Gesztesy, A. Kiselev, and K. A. Makarov, Uniqueness results for matrix-valued Schrödinger, Jacobi, and Dirac-type operators, Math. Nachr., to appear.

[43] F. Gesztesy, M. Krishna, and G. Teschl, On isospectral sets of Jacobi operators, Commun. Math. Phys. 181, 631-645 (1996). MR 97i:47048

[44] F. Gesztesy and K. A. Makarov, Some applications of the spectral shift operator, in Operator Theory and its Applications, A. G. Ramm, P. N. Shivakumar, and A. V.Strauss (eds.), Fields Institute Communications, Vol. 25, Amer. Math. Society, Providence RI, 2000, pp. 267-292. MR 2001f: 47018

[45] F. Gesztesy and K. A. Makarov, The $\Xi$ operator and its relation to Krein's spectral shift function, J. Analyse Math. 81, 139-183 (2000). MR 2001i:47016

[46] F. Gesztesy, K. A. Makarov, and S. N. Naboko, The spectral shift operator, in Mathematical Results in Quantum Mechanics, J. Dittrich, P. Exner, and M. Tater (eds.), Operator Theory: Advances and Applications, Vol. 108, Birkhäuser, Basel, 1999, pp. 59-90. MR 2000k:47012

[47] F. Gesztesy, K. A. Makarov, and E. Tsekanovskii, An Addendum to Krein's Formula, J. Math. Anal. Appl. 222, 594-606 (1998). MR 99g:47047

[48] F. Gesztesy, W. Schweiger, and B. Simon, Commutation methods applied to the mKdVequation, Trans. Amer. Math. Soc. 324, 465-525 (1991). MR 92b:35132

[49] F. Gesztesy and B. Simon, The $\xi$ function, Acta Math. 176, 49-71 (1996). MR 97e:47078

[50] F. Gesztesy and B. Simon, A new approach to inverse spectral theory, II. General real potentials and the connection to the spectral measure, Ann. of Math. 152, 593-643 (2000). MR 2001m:34185b

[51] F. Gesztesy and B. Simon, On local Borg-Marchenko uniqueness results, Commun. Math. Phys. 211, 273-287 (2000). MR 2001b:34020

[52] F. Gesztesy and E. Tsekanovskii, On matrix-valued Herglotz functions, Math. Nachr. 218, 61-138 (2000). MR 2001j:47018

[53] F. Gesztesy and R. Weikard, A characterization of all elliptic algebro-geometric solutions of the AKNS hierarchy, Acta Math. 181, 63-108 (1998). MR 99k:14052

[54] R. Giachetti and R. A. Johnson, Spectral theory of second-order almost periodic differential operators and its relation to classes of nonlinear evolution equations, Nuovo Cim. 82B, 125-168 (1984). MR 86j:58127

[55] B. Grebert, Inverse scattering for the Dirac operator on the real line, Inverse Problems 8 , 787-807 (1992). MR 93j:34021

[56] B. Grebert and J.-C. Guillot, Gaps of one-dimensional periodic AKNS systems, Forum Math. 5, 459-504 (1993). MR 95d:34135

[57] B. J. Harris, The asymptotic form of the Titchmarsh-Weyl m-function associated with a Dirac system, J. London Math. Soc. (2), 31, 321-330 (1985). MR 87e:34032

[58] E. Hille, Green's transforms and singular boundary value problems, J. Math. Pures Appl. (9), 42, 331-349 (1963). MR 28:3191

[59] D. B. Hinton, private communication, September, 1999.

[60] D. B. Hinton, A. K. Jordan, M. Klaus, and J. K. Shaw, Inverse scattering on the line for a Dirac system, J. Math. Phys. 32, 3015-3030 (1991). MR 93b:34110

[61] D. B. Hinton, M. Klaus, and J. K. Shaw, Asymptotic phase, asymptotic modulus, and Titchmarsh-Weyl coefficient for a Dirac system, J. Math. Anal. Appl. 142, 108-129 (1989). MR 90e:34046

[62] D. B. Hinton, M. Klaus, and J. K. Shaw, Series representation and asymptotics for Titchmarsh-Weyl m-functions, Diff. Integral Eqs. 2, 419-429 (1989). MR 90m:34057

[63] D. B. Hinton and J. K. Shaw, On Titchmarsh-Weyl $M(\lambda)$-functions for linear Hamiltonian systems, J. Diff. Eqs. 40, 316-342 (1981). MR 82h:34018

[64] D. B. Hinton and J. K. Shaw, On the spectrum of a singular Hamiltonian system, Quaest. Math. 5, 29-81 (1982). MR 83b:34024

[65] D. B. Hinton and J. K. Shaw, Hamiltonian systems of limit point or limit circle type with both endpoints singular, J. Diff. Eqs. 50, 444-464 (1983). MR 85b:58044

[66] D. B. Hinton and J. K. Shaw, On boundary value problems for Hamiltonian systems with two singular points, SIAM J. Math. Anal. 15, 272-286 (1984). MR 87a:34021

[67] D. B. Hinton and J. K. Shaw, On the spectrum of a singular Hamiltonian system, II, Quaest. Math. 10, 1-48 (1986). MR 87k:34029 
[68] D. B. Hinton and A. Schneider, On the Titchmarsh-Weyl coefficients for singular SHermitian Systems I, Math. Nachr. 163, 323-342 (1993). MR 94j:34027

[69] D. B. Hinton and A. Schneider, On the Titchmarsh-Weyl coefficients for singular SHermitian Systems II, Math. Nachr. 185, 67-84 (1997). MR 98h:34048

[70] H. Hochstadt, On the determination of a Hill's equation from its spectrum, Arch. Rat. Mech. Anal. 19, 353-362 (1965). MR 31:6019

[71] R. A. Johnson, The recurrent Hill's equation, J. Diff. Eqs. 46, 165-193 (1982). MR 84m:34037

[72] R. A. Johnson, m-Functions and Floquet exponents for linear differential systems, Ann. Mat. Pura Appl., Ser. 4, 147, 211-248 (1987). MR 88m:34021

[73] R. Johnson and J. Moser, The rotation number for almost periodic potentials, Commun. Math. Phys. 84, 403-438 (1982). MR 83h:34018

[74] R. Johnson, S. Novo, and R. Obaya, Ergodic properties and Weyl M-functions for random linear Hamiltonian systems, Proc. Roy. Soc. Edinburgh 130A, 1045-1079 (2000).

[75] V. I. Kogan and F. S. Rofe-Beketov, On square-integrable solutions of symmetric systems of differential equations of arbitrary order, Proc. Roy. Soc. Edinburgh 74A, 1-40 (1974). MR 56:12392

[76] S. Kotani, Ljapunov indices determine absolutely continuous spectra of stationary random one-dimensional Schrödinger operators, in Stochastic Analysis, K. Itǒ (ed.), North-Holland, Amsterdam, 1984, pp. 225-247. MR 86h:60117

[77] S. Kotani, One-dimensional random Schrödinger operators and Herglotz functions, in "Probabilistic Methods in Mathematical Physics", K. Itǒ and N. Ikeda (eds.), Academic Press, New York, 1987, p. 219-250. MR 89h:60100

[78] S. Kotani and M. Krishna, Almost periodicity of some random potentials, J. Funct. Anal. 78, 390-405 (1988). MR 89i:60133

[79] S. Kotani and B. Simon, Stochastic Schrödinger operators and Jacobi matrices on the strip, Commun. Math. Phys. 119, 403-429 (1988). MR 90e:82010

[80] A. M. Krall, $M(\lambda)$ theory for singular Hamiltonian systems with one singular point, SIAM J. Math. Anal. 20, 664-700 (1989). MR 91c:34036

[81] A. M. Krall, $M(\lambda)$ theory for singular Hamiltonian systems with two singular points, SIAM J. Math. Anal. 20, 701-715 (1989). MR 91c:34037

[82] M. Lesch and M. Malamud, The inverse spectral problem for first order systems on the half line, Operator Theory: Advances and Applications, Vol. 117, Birkhäuser, Basel, 2000, p. 199-238. MR 2001h:34116

[83] M. Lesch and M. Malamud, On the number of square integrable solutions and self-adjointness of symmetric first order systems of differential equations, Spectral and Evolution Problems, Vol. II (Sevastopol, 2000), Natl. Taurida Univ., Simferopol, 2001, pp. 150-167.

[84] B. M. Levitan and M. Otelbaev, Conditions for self-adjointness of the Schrödinger and Dirac operators, Trans. Moscow. Math. Soc. 42, 139-156 (1982). MR 82m:47034

[85] B. M. Levitan and I. S. Sargsjan, Introduction to Spectral Theory, Amer. Math. Soc., Providence, R. I., 1975. MR 51:6026

[86] B. M. Levitan and I. S. Sargsjan, Sturm-Liouville and Dirac Operators, Kluwer, Dordrecht, 1991. MR 92i:34119

[87] M. M. Malamud, Similarity of Volterra operators and related questions of the theory of differential equations of fractional order, Trans. Moscow Math. Soc. 55, 57-122 (1994). MR 98e:47051

[88] M. M. Malamud, A connection between the potential matrix of the Dirac system and its Wronskian, Dokl. Math. 52, 296-299 (1995). MR 98j:34019

[89] M. M. Malamud, Uniqueness questions in inverse problems for systems of ordinary differential equations on a finite interval, Trans. Moscow Math. Soc. 60, 173-224 (1999). MR 2001h:34026

[90] M. M. Malamud, Borg type theorems for first-order systems on a finite interval, Funct. Anal. Appl. 33, 64-68 (1999). MR 2000j:34021

[91] Yu. I. Manin, Matrix solitons and bundles over curves with singularities, Funct. Anal. Appl. 12, 286-295 (1978). MR 80g:35114

[92] V. A. Marchenko, Certain problems in the theory of second-order differential operators, Doklady Akad. Nauk SSSR 72, 457-460 (1950) (Russian). MR 12:183c 
[93] V. A. Marčenko, Some questions in the theory of one-dimensional linear differential operators of the second order. I, Trudy Moskov. Mat. Obšč. 1, 327-420 (1952) (Russian); English transl. in Amer. Math. Soc. Transl. (2) 101, 1-104 (1973). MR 15:315b

[94] V. A. Marchenko, Sturm-Liouville Operators and Applications, Birkhäuser, Basel, 1986. MR 88f:34034

[95] V. A. Marchenko, Nonlinear Equations and Operator Algebras, Reidel, Dordrecht, 1988. MR 89j:35003

[96] V. V. Martynov, Condition for discreteness and continuity of the spectrum of a selfadjoint system of first-order differential equations, Sov. Math. Dokl. 6, 1546-1550 (1965). MR 33:1762

[97] R. Mennicken, A.L. Sakhnovich, and C. Tretter, Direct and inverse spectral problem for a system of differential equations depending rationally on the spectral parameter, Duke Math. J. 109, 413-449 (2001).

[98] T. V. Misyura, Asymptotic formula for the Weyl solutions of the Dirac equations, Dokl. Akad. Nauk Ukrain. SSR 5, 26-28 (1991). (Russian) MR 92g:34113

[99] K. N. Murty, K. R. Prasad, and M. A. S. Srinivas, Upper and lower bounds for the solution of the general matrix Riccati differential equation, J. Math. Anal. Appl. 147, 12-21 (1990). MR 91a:34013

[100] S. A. Orlov, Nested matrix disks analytically depending on a parameter, and theorems on the invariance of ranks of radii of limiting disks, Math. USSR Izv. 10, 565-613 (1976). MR 54:13625

[101] G. Pólya and G. Szegő, Problems and Theorems in Analysis I, Springer, Berlin, 1972. MR 49:8782

[102] A. Rybkin, KdV invariants and Herglotz functions, Diff. Integral Eqs. 14, 493-512 (2001). MR 2001k:34155

[103] A. Rybkin, On the trace approach to the inverse scattering problem in dimension one, SIAM J. Math. Anal. 32, 1248-1264 (2001).

[104] A. L. Sakhnovich, Asymptotic behavior of spectral functions of an $S$-node, Sov. Math. (Iz. VUZ) 32, No. 9, 92-105 (1988). MR 90f:47025

[105] A. L. Sakhnovich, Nonlinear Schrödinger equation on a semi-axis and an inverse problem associated with it, Ukrain. Math. J. 42, 316-323 (1990). MR 91c:34118

[106] A. L. Sakhnovich, Spectral functions of a canonical system of order $2 n$, Math. USSR Sbornik 71, 355-369 (1992). MR 92c:47056

[107] A. Sakhnovich, Canonical systems and transfer matrix-functions, Proc. Amer. Math. Soc. 125, 1451-1455 (1997). MR 97g:34108

[108] A. Sakhnovich, Dirac type and canonical systems: spectral functions, direct and inverse problems, preprint, 2001.

[109] L. A. Sakhnovich, Evolution of spectral data and nonlinear equations, Ukrain. Math. J. 40, 459-461 (1988). MR 90g:35150

[110] L. A. Sakhnovich, Inverse problems for equations systems, in Matrix and Operator Valued Functions: The Vladimir Petrovich Potapov Memorial Volume, I. Gohberg and L. A. Sakhnovich (eds.), Operator Theory: Advances and Applications, Vol. 72, Birkhäuser, Basel, 1994, pp. 202-211. MR 95k:34022

[111] L. A. Sakhnovich, Method of operator identities and problems of analysis, St. Petersburg Math. J. 5, 1-69 (1994). MR 95f:47002

[112] L. A. Sakhnovich, Spectral analysis of a class of canonical differential systems, St. Petersburg Math. J. 10, 147-158 (1999). MR 99j:34120

[113] L. A. Sakhnovich, Spectral Theory of Canonical Differential Systems. Method of Operator Identities, Operator Theory: Advances and Applications, Vol. 107, Birkhäuser, Basel, 1999. MR 2000e: 47073 .

[114] Yu. L. Shmul'yan, On operator R-functions, Siberian Math. J. 12, 315-322 (1971). MR 45:949

[115] B. Simon, A new aproach to inverse spectral theory, I. Fundamental formalism, Ann. of Math. 150, 1029-1057 (1999). MR 2001m:34185a

[116] M. Sodin and P. Yuditskii, Almost periodic Sturm-Liouville operators with Cantor homogeneous spectrum, Comment. Math. Helvetici 70, 639-658 (1995). MR 96k:34175

[117] S. Timischl, A trace formula for one-dimensional Dirac operators, diploma thesis, Department of Mathematics, University of Missouri-Columbia, 1995. 
[118] E. C. Titchmarsh, Introduction to the Theory of Fourier Integrals, Chelsea, New York, 1986. MR 89c: 42002

[119] J. Weidmann, Oszillationsmethoden für Systeme gewöhnlicher Differentialgleichungen, Math. Z. 119, 349-373 (1971). MR 44:2975

[120] H. Weyl, Über gewöhnliche Differentialgleichungen mit Singularitäten und die zugehörigen Entwicklungen willkürlicher Funktionen, Math. Ann. 68, 220-269 (1910).

Department of Mathematics and Statistics, University of Missouri-Rolla, Rolla, Missouri 65409

E-mail address: sclark@umr.edu

URL: http://www.umr.edu/ ${ }^{\sim}$ clark

Department of Mathematics, University of Missouri, Columbia, Missouri 65211

E-mail address: fritz@math.missouri.edu

$U R L:$ http://www.math.missouri.edu/people/fgesztesy.html 